DisCRETE ANALYSIS, 2016:6, 48 pp.

www.discreteanalysisjournal.com

\title{
Quantitative Affine Approximation for UMD Targets
}

\author{
Tuomas Hytönen* $\quad$ Sean $\mathrm{Li}^{\dagger} \quad$ Assaf Naor ${ }^{\ddagger}$
}

Received 5 November 2015; Published 28 February 2016

\begin{abstract}
It is shown here that if $\left(Y,\|\cdot\|_{Y}\right)$ is a Banach space in which martingale differences are unconditional (a UMD Banach space) then there exists $c=c(Y) \in(0, \infty)$ with the following property. For every $n \in \mathbb{N}$ and $\varepsilon \in(0,1 / 2]$, if $\left(X,\|\cdot\|_{X}\right)$ is an $n$-dimensional normed space with unit ball $B_{X}$ and $f: B_{X} \rightarrow Y$ is a 1-Lipschitz function then there exists an affine mapping $\Lambda: X \rightarrow Y$ and a sub-ball $B^{*}=y+\rho B_{X} \subseteq B_{X}$ of radius $\rho \geqslant \exp \left(-(1 / \varepsilon)^{c n}\right)$ such that $\|f(x)-\Lambda(x)\|_{Y} \leqslant \varepsilon \rho$ for all $x \in B^{*}$. This estimate on the macroscopic scale of affine approximability of vector-valued Lipschitz functions is an asymptotic improvement (as $n \rightarrow \infty$ ) over the best previously known bound even when $X$ is $\mathbb{R}^{n}$ equipped with the Euclidean norm and $Y$ is a Hilbert space.
\end{abstract}

Key words and phrases: Quantitative differentiation, unconditional martingale differences, LittlewoodPaley theory

\section{Introduction}

In what follows, the unit ball of a normed space $\left(X,\|\cdot\|_{X}\right)$ is denoted $B_{X} \stackrel{\text { def }}{=}\left\{x \in X:\|x\|_{X}<1\right\}$. For $p \in[1, \infty]$ and $n \in \mathbb{N}$, the space $\mathbb{R}^{n}$ equipped with the $\ell_{p}$ norm is denoted as usual by $\ell_{p}^{n}$. Given two metric spaces $\left(U, d_{U}\right)$ and $\left(V, d_{V}\right)$, the Lipschitz constant of a mapping $f: U \rightarrow V$ is denoted $\|f\|_{\text {Lip. }}$. Throughout this article, given $a, b \in(0, \infty)$, the notations $a \lesssim b$ and $b \gtrsim a$ mean that $a \leqslant c b$ for some universal constant $c \in(0, \infty)$. The notation $a \asymp b$ stands for $(a \lesssim b) \wedge(b \lesssim a)$.

${ }^{*}$ Supported by ERC grant ANPROB and by the Academy of Finland through projects 130166, 133264 and CoE in Analysis and Dynamics.

$\dagger$ Supported by NSF grants CCF-0832795 and DMS-1303910.

$\ddagger$ Supported by NSF grant CCF-0832795, BSF grant 2010021, the Packard Foundation and the Simons Foundation. 
For $n \in \mathbb{N}$ and $\varepsilon \in(0,1)$ let $r_{n}(\varepsilon) \geqslant 0$ be the supremum over those $r \in[0,1)$ such that for every Lipschitz function $f: B_{\ell_{2}^{n}} \rightarrow \ell_{2}$ there exists a linear mapping $T: \ell_{2}^{n} \rightarrow \ell_{2}$, a vector $a \in \ell_{2}$, and a sub-ball $B^{*}=x_{0}+\rho B_{\ell_{2}^{n}} \subseteq B_{\ell_{2}^{n}}$ of radius $\rho \geqslant r$, such that

$$
\forall x \in B^{*}, \quad \frac{\|f(x)-(a+T x)\|_{\ell_{2}}}{\rho} \leqslant \varepsilon\|f\|_{\text {Lip }} .
$$

Thus, all the Hilbert space-valued 1-Lipschitz functions on the Euclidean unit ball of $\mathbb{R}^{n}$ are guaranteed to be $\varepsilon$-close to some affine function on some sub-ball of radius at least $r_{n}(\varepsilon)$, where $\varepsilon$-closeness is measured relative to the scale of the sub-ball. A lower bound on $r_{n}(\varepsilon)$ corresponds to a differentiationtype theorem asserting that any such function is macroscopically close to being affine rather than being infinitesimally affine. Crucially, the macroscopic lower bound on the scale of affine approximability is independent of the given function.

The basic question in which we are interested is that of determining the asymptotic behavior of $r_{n}(\varepsilon)$ as $n \rightarrow \infty$. Qualitatively, we ask for an asymptotic understanding of those Hilbert space-valued Lipschitz functions on $B_{\ell_{2}^{n}}$ that are hardest to approximate by affine functions.

There is a big gap between the known upper and lower bounds on $r_{n}(\varepsilon)$. We have $r_{n}(\varepsilon) \leqslant e^{-c n / \varepsilon^{2}}$, where $c \in(0, \infty)$ is a universal constant; see Section 2.2 below. The only known lower bound [57] on $r_{n}(\varepsilon)$ is $r_{n}(\varepsilon) \geqslant e^{-(n / \varepsilon)^{C n}}$, where $C \in(0, \infty)$ is a universal constant. For concreteness, by choosing, say, $\varepsilon=\frac{1}{4}$ and denoting $r_{n}=r_{n}\left(\frac{1}{4}\right)$, the best known bounds on $r_{n}$ become

$$
e^{-n^{K n}} \leqslant r_{n} \leqslant e^{-\kappa n}
$$

for some universal constants $\kappa, K \in(0, \infty)$. An illustrative special case of the main result that is obtained here (to be described in full below) is the following asymptotic improvement over the leftmost inequality (2), which holds for every $n \in \mathbb{N}$ and for some universal constant $K \in(0, \infty)$.

$$
r_{n} \geqslant e^{-e^{K n}} \text {. }
$$

\subsection{The modulus of $L_{p}$ affine approximabilty}

Despite the fact that the above question was phrased in the context of Hilbert spaces, a setting which arguably best highlights its fundamental nature, it is important to study it in the context of mappings between more general normed spaces; it is in this setting, for example, that it becomes relevant to Bourgain's discretization problem [13, 35], as explained in [57, Section 1.1] (see Remark 3 below).

Definition 1. Fix $n \in \mathbb{N}$ and let $\left(X=\mathbb{R}^{n},\|\cdot\|_{X}\right)$ be an n-dimensional Banach space. Also, let $\left(Y,\|\cdot\|_{Y}\right)$ be an inifinite dimensional Banach space. For $p \in(0, \infty]$ and $\varepsilon \in(0,1)$ define $r_{p}^{X \rightarrow Y}(\varepsilon)$ to be the supremum over those $r \geqslant 0$ with the following property. For every Lipschitz function $f: B_{X} \rightarrow Y$ there exists $y \in X$ and $\rho \in[r, \infty)$ such that $y+\rho B_{X} \subseteq B_{X}$, and there exists $a \in Y$ and a linear operator $T: X \rightarrow Y$ whose operator norm satisfies $\|T\|_{X \rightarrow Y} \leqslant 3\|f\|_{\text {Lip }}$, such that

$$
\left(\frac{1}{\operatorname{vol}\left(x+\rho B_{X}\right)} \int_{y+\rho B_{X}}\|f(z)-(a+T z)\|_{Y}^{p} \mathrm{~d} z\right)^{\frac{1}{p}} \leqslant \varepsilon \rho\|f\|_{\text {Lip }} .
$$

We call $r_{p}^{X \rightarrow Y}(\cdot)$ the modulus of $L_{p}$ affine approximability corresponding to $X$ and $Y$. 
Using the notation of Definition 1, the quantity $r_{n}(\varepsilon)$ that we defined above can be written as

$$
r_{n}(\varepsilon) \stackrel{\text { def }}{=} r_{\infty}^{\ell_{2}^{n} \rightarrow \ell_{2}}(\varepsilon)
$$

Indeed, the $L_{\infty}$ requirement (1) implies that $\|T\|_{\ell_{2}^{n} \rightarrow \ell_{2}} \leqslant(1+2 \varepsilon)\|f\|_{\text {Lip }} \leqslant 3\|f\|_{\text {Lip. }}$. For finite $p$, the $L_{p}$ bound (4) does not automatically imply a bound on $\|T\|_{X \rightarrow Y}$, which is the reason why we added the requirement $\|T\|_{X \rightarrow Y} \leqslant 3\|f\|_{\text {Lip }}$ as part of the definition of $r_{p}^{X \rightarrow Y}(\varepsilon)$.

The case $p=\infty$ of Definition 1, for which we shall use below the simpler notation

$$
r^{X \rightarrow Y}(\varepsilon) \stackrel{\text { def }}{=} r_{\infty}^{X \rightarrow Y}(\varepsilon)
$$

was introduced by Bates, Johnson, Lindenstrauss, Preiss and Schechtman [6], who proved that $r^{X \rightarrow Y}(\varepsilon)>$ 0 for all $\varepsilon \in(0,1)$ if and only if $Y$ admits an equivalent uniformly convex norm; see [6] for beautiful geometric applications of this result. The best known lower bound on $r^{X \rightarrow Y}(\varepsilon)$ (in terms of $n, \varepsilon$ and the modulus of uniform convexity of $Y$ ) was obtained in [57]. This bound is

$$
r^{X \rightarrow Y}(\varepsilon) \geqslant e^{-(n / \varepsilon)^{c(Y) n}}
$$

where $c(Y) \in(0, \infty)$ depends only the modulus of uniform convexity of the target Banach space $\left(Y,\|\cdot\|_{Y}\right)$. An explicit estimate on $c(Y)$ appears in [57, Thm. 1.1]. Here we obtain an estimate that is asymptotically better than (5) as $n \rightarrow \infty$ provided that $Y$-valued martingale differences are unconditional ( $Y$ is a UMD Banach space). Note that all the classical reflexive Banach spaces have this property, but one can construct [67] uniformly convex Banach spaces that are not UMD.

Formally, a Banach space $\left(Y,\|\cdot\|_{Y}\right)$ is said to be a UMD Banach space if there exists $\beta \in(1, \infty)$ such that if $\left\{M_{j}\right\}_{j=0}^{\infty}$ is a $Y$-valued square-integrable martingale defined on some probability space $(\Omega, \mathbb{P})$ then for every $n \in \mathbb{N}$ and every choice of signs $\varepsilon_{1}, \ldots, \varepsilon_{n} \in\{-1,1\}$ we have

$$
\int_{\Omega}\left\|M_{0}+\sum_{j=1}^{n} \varepsilon_{n}\left(M_{j}-M_{j-1}\right)\right\|_{Y}^{2} \mathrm{~d} \mathbb{P} \leqslant \beta^{2} \int_{\Omega}\left\|M_{n}\right\|_{Y}^{2} \mathrm{~d} \mathbb{P}
$$

If $Y$ is a UMD Banach space then the infimum over those $\beta \in(1, \infty)$ for which (6) holds true for every square-integrable $Y$-valued martingale $\left\{M_{j}\right\}_{j=0}^{\infty}$ is denoted below by $\beta(Y)$. Examples of UMD Banach spaces include all $L_{p}(\mu)$ spaces when $p \in(1, \infty)$, in which case $\beta\left(L_{p}(\mu)\right) \asymp p^{2} /(p-1)$. See [14] and the references therein for more information on UMD spaces.

Theorem 2 below asserts an improved lower bound on the modulus of affine approximability $r^{X \rightarrow Y}(\varepsilon)$, provided that $Y$ is a UMD space.

Theorem 2. There is a universal constant $c \in[1, \infty)$ such that for every $n \in \mathbb{N}$ and $\beta \in[2, \infty)$, if $\left(X,\|\cdot\|_{X}\right)$ is an n-dimensional normed space and $\left(Y,\|\cdot\|_{Y}\right)$ is a UMD Banach space satisfying $\beta(Y) \leqslant \beta$ then for every $\varepsilon \in(0,1 / 2)$ we have

$$
r^{X \rightarrow Y}(\varepsilon) \geqslant \exp \left(-\frac{(\beta n)^{c \beta}}{\varepsilon^{c(n+\beta)}}\right) .
$$


Remark 3. By substituting Theorem 2 into equation (12) of [57] one obtains a bound on Bourgain's discretization modulus in the special case of UMD targets that improves over the bound that was deduced in [57, Section 1.1] and matches Bourgain's original bound [13]. Specifically, one obtains the refined estimate that appears in equation (2) of [35]. This yields a new proof of the best known general bound in Bourgain's discretization problem via an approach that is entirely different from Bourgain's method, albeit in the special (though still very general) case of UMD targets. We note that due to the recent progress in [35] a stronger bound is available here when the target is $L_{p}$.

The main reason why we study here the modulus of $L_{p}$ affine approximability $r_{p}^{X \rightarrow Y}(\varepsilon)$ is that it relates to $r^{X \rightarrow Y}(\varepsilon)$ through Lemma 4 below, whose simple proof appears in Section 2.1. The advantage of working with finite $p$ is that it allows us to use a variety of analytic tools, such as vector-valued Littlewood-Paley theory and complex interpolation.

Lemma 4. Fix $n \in \mathbb{N}$ and $p \in[1, \infty)$. Suppose that $\left(X,\|\cdot\|_{X}\right)$ and $\left(Y,\|\cdot\|_{Y}\right)$ are Banach spaces with $\operatorname{dim}(X)=n$. Then for every $\varepsilon \in(0,1)$ we have

$$
r^{X \rightarrow Y}(\varepsilon) \geqslant r_{p}^{X \rightarrow Y}\left(\left(\frac{\varepsilon}{9}\right)^{1+\frac{n}{p}}\right) .
$$

Due to Lemma 4, Theorem 2 is a consequence of Theorem 5 below, which is our main result. Its proof is based on a vector-valued variant of an argument of Dorronsoro [26], combined with a wide variety of additional analytic and geometric ingredients of independent interest.

Theorem 5. There exist universal constants $\kappa, C \in[1, \infty)$ such that for every $\beta \in[2, \infty)$ and $n \in \mathbb{N}$, if $\left(X,\|\cdot\|_{X}\right)$ is an $n$-dimensional normed space and $\left(Y,\|\cdot\|_{Y}\right)$ is a UMD Banach space satisfying $\beta(Y) \leqslant \beta$, then for every $\varepsilon \in(0,1)$ we have

$$
r_{\kappa \beta}^{X \rightarrow Y}(\varepsilon) \geqslant \exp \left(-\frac{(\beta n)^{C \kappa \beta}}{\varepsilon^{\kappa \beta}}\right) .
$$

\subsection{Previous work}

Over the past several decades, research on quantitative differentiation has proceeded roughly along two lines of inquiry, one of which arising from functional analysis and metric geometry and the other arising from rectifiability questions in harmonic analysis. The present work belongs to the former direction, but its main contribution is the use of methods from the latter direction in this new context while incorporating various Banach space theoretic tools.

Bates, Johnson, Lindenstrauss, Preiss and Schechtman studied [6] quantitative differentiation in order to prove the rigidity of certain classes of Banach spaces under nonlinear quotients. The same notion was used in [57] for metric embeddings, namely as an alternative approach to Bourgain's discretization problem [13] when the target Banach space is uniformly convex. The methods in this context fall under the category of (extensions of) "approximate midpoint arguments," as initiated by Enflo [7] to prove that $L_{1}$ is not uniformly homeomorphic to $\ell_{1}$, and further developed in [13, 27, 46, 65] (see also Chapter 10 of [8]). As examples of the many related applications to quantitative embedding theory and rigidity questions, see also [60, 54, 20, 27, 19, 64, 55, 56]. 
QuANTITATIVE AFFine APPROXIMATION For UMD TARgETS

Parallel developments of a different nature arose in harmonic analysis, as part of the quest to develop a quantitative theory of rectifiability, with applications to singular integrals. Notable contributions along these lines include classical works of Stein (see the monograph [72]), through the works of Dorronsoro [26], Jones [48, 49], David and Semmes [22, 23, 24], as well as the more recent work of Azzam and Schul [2]. The work of Dorronsoro [26] directly influenced the present article, and we were also greatly inspired by the works of David and Semmes [22, 23, 24]. These works introduced and studied quantities that correspond to Definition 1 when $X$ is the Euclidean space $\mathbb{R}^{n}$ and $Y$ is the real line. Such methods also yield results for mappings from $\mathbb{R}^{n}$ to $\mathbb{R}^{m}$, but with statements that include implicit parameters that are allowed to depend on $m, n$. In [24] David and Semmes compare their work to that of Bates, Johnson, Lindenstrauss, Preiss and Schechtman [6], noting that the latter methods are different, and even yield results for infinite dimensional spaces.

Our contribution here follows the harmonic-analytic methodology, while overcoming several difficulties. Firstly, the literature on quantitative rectifiability ignores the dependence on the dimension $n$, while this dependence is the main topic of interest in the present context. In fact, a direct examination of the dependence on $n$ that is implicit in the above cited works reveals that it is insufficient for the purpose of obtaining improved bounds on $r^{X \rightarrow Y}(\varepsilon)$. Secondly, in our setting the domain $X$ is a general $n$-dimensional normed space $X$ rather than a Euclidean space, and our arguments address this point. A final important difference is that we treat infinite dimensional Banach spaces $Y$ as targets. Overcoming this requires substantial effort, because the infinite dimensional arguments of Bates, Johnson, Lindenstrauss, Preiss and Schechtman [6] do not seem to be applicable in our setting. The present work yields an infinite dimensional version of an inequality that Dorronsoro obtained [26] for real-valued functions. Such an infinite dimensional extension of Dorronsoro's work is not routine, and in particular it does not hold true for arbitrary infinite dimensional Banach space targets $Y$. In fact, the geometry of $Y$ influences the structure of the inequality thus obtained, while stronger inequalities hold true for real-valued functions. The assumption that $Y$ is UMD is used several times in our argument through a rich UMD-valued Fourier-analytic toolkit that has been developed by many authors over the past four decades.

\subsection{Open questions}

We list below some open questions that arise naturally from our work.

Question 6 (Asymptotics of $r^{X \rightarrow Y}(\varepsilon)$ ). Obviously, the most tantalizing open question in the present context is to determine the rate at which $r_{n}$ tends to 0 as $n \rightarrow \infty$, even roughly: say, is this rate exponential, doubly exponential, or of some intermediate behavior? More importantly for potential applications, it remains open to obtain sharp bounds on the quantity $r^{X \rightarrow Y}(\varepsilon)$ when $X$ is a finite dimensional Banach space, $Y$ is a uniformly convex Banach space or belongs to some important class of Banach spaces (e.g. UMD spaces or uniformly convex lattices), and $\varepsilon \in(0,1)$.

Question 7 (Infinite dimensional domains). The question of characterizing those pairs of Banach space $X, Y$ for which $r^{X \rightarrow Y}(\varepsilon)>0$ for every $\varepsilon \in(0,1)$ was solved in [6] also when $\operatorname{dim}(X)=\infty$ and $\operatorname{dim}(Y)<\infty$ : this happens if and only if $X$ admits an equivalent uniformly smooth norm. It remains open to understand the asymptotic behavior of $r^{X \rightarrow Y}(\varepsilon)$ in the setting of uniformly smooth infinite dimensional domains and finite dimensional ranges. In particular, the rate at which $r^{\ell_{2} \rightarrow \ell_{2}^{n}}\left(\frac{1}{4}\right)$ tends to 0 as $n \rightarrow \infty$ is unknown. An 
explicit lower bound on $r^{X \rightarrow Y}(\varepsilon)$ in terms of the modulus of uniform smoothness of $X$ and $\operatorname{dim}(Y)$ can be deduced from an examination of the proof in [6], but we believe that this bound is far from being optimal.

Question 8 (Uniformly convex targets). As stated earlier, by [6] we know that if $\operatorname{dim}(X)=n<\infty$ and $\operatorname{dim}(Y)=\infty$ then $r^{X \rightarrow Y}(\varepsilon)>0$ for every $\varepsilon \in(0,1)$ if and only if $Y$ admits and equivalent uniformly convex norm. However, the best known lower bounds on $r^{X \rightarrow Y}(\varepsilon)$ in this (maximal) generality remain those of [57], and these bounds are weaker than Theorem 2 (as $n \rightarrow \infty)$, which does not cover all uniformly convex ranges $Y$ because Pisier [67] proved that there exist uniformly convex Banach spaces that are not UMD (even such uniformly convex Banach lattices exist, as shown by Bourgain in [11]; see also the recent example by Qiu [69]). It would be interesting to obtain an improved bound as in Theorem 2 under the weaker assumption that $Y$ is uniformly convex. Our proof of Theorem 2 definitely uses properties of $Y$ that imply the UMD property (e.g., we rely on the boundedness of the $Y$-valued Hilbert transform, which was shown by Bourgain [11] to imply that $Y$ is UMD). However, the conclusion of Theorem 2, or even Theorem 5, may be valid when $Y$ is uniformly convex, and the same holds true for some of our other results, such as Theorem 19 below. Certain aspects of vector-valued Littelwood-Paley theory are known to hold true for uniformly convex targets (see e.g. [59]), so it would be interesting to investigate the extent to which the UMD property is needed for our results. If, on the other hand, Theorem 5 or Theorem 19 imply the UMD property then this would be a new characterization of UMD spaces.

Question 9 (Asymptotics of $r_{p}^{X \rightarrow Y}(\varepsilon)$ for finite $p$ ). It would be interesting to understand the asymptotic behavior of the modulus of $L_{p}$ affine approximability $r_{p}^{X \rightarrow Y}(\varepsilon)$, even in the special case when $X$ and $Y$ are both Hilbert spaces, $p=2$ and, say, $\varepsilon=1 / 2$. A careful examination of the proof of Theorem 5 in this Hilbertian setting (in which case some of the steps that we perform below are not needed, and several estimates can be easily improved) reveals that for some $c \in(0, \infty)$,

$$
\forall n \in \mathbb{N}, \forall \varepsilon \in(0,1), \quad r_{2}^{\ell_{2}^{n} \rightarrow \ell_{2}}(\varepsilon) \gtrsim e^{-\frac{c(n \log n)^{2}}{\varepsilon^{2}}} .
$$

We do not know the extent to which (7) is best possible; it seems plausible that with more work one could improve the dependence on $n$ in the exponent, but we do not presently have an upper bound that comes close to the lower bound in (7). Note that while the moduli $r_{p}^{X \rightarrow Y}(\varepsilon)$ are interesting in their own right, we do not have geometric applications of them as in the case $p=\infty$. So, as a more amorphous research direction, it would be interesting to find geometric applications of bounds on $r_{p}^{X \rightarrow Y}(\varepsilon)$ (other than as a tool to bound $r_{\infty}^{X \rightarrow Y}(\varepsilon)$, which is the application that we present here).

\section{Geometric preliminaries}

Fix from now on an integer $n$ and an $n$-dimensional normed space $\left(X,\|\cdot\|_{X}\right)$. We shall also fix a normed space $\left(Y,\|\cdot\|_{Y}\right)$. In later sections we will need $Y$ to be a UMD Banach space, but the statements of the present section hold true when $Y$ is a general normed space.

By John's theorem [45] there exists a scalar product $\langle\cdot, \cdot\rangle$ on $X$ with respect to which we can identify $X$ with $\mathbb{R}^{n}$ and we have

$$
\forall x \in \mathbb{R}^{n}, \quad\|x\|_{2} \leqslant\|x\|_{X} \leqslant \sqrt{n} \cdot\|x\|_{2},
$$


where $\|\cdot\|_{2}=\|\cdot\|_{\ell_{2}^{n}}$. We shall also use the standard notation $B^{n} \stackrel{\text { def }}{=} B_{\ell_{2}^{n}}$ and $S^{n-1} \stackrel{\text { def }}{=} \partial B^{n}$. This Euclidean structure will be fixed from now on. Despite the fact that $X$ is now endowed with two metrics (those induced by $\|\cdot\|_{X}$ and $\|\cdot\|_{2}$ ), we shall tacitly maintain throughout the ensuing discussion the convention that whenever $\Omega \subseteq \mathbb{R}^{n}$ and $f: \Omega \rightarrow Y$ then $\|f\|_{\operatorname{Lip}(\Omega)}$ denotes the Lipschitz constant of $f$ with respect to the metric induced by $\|\cdot\|_{X}$, i.e.,

$$
\|f\|_{\operatorname{Lip}(\Omega)} \stackrel{\text { def }}{=} \sup _{\substack{x, y \in \Omega \\ x \neq y}} \frac{\|f(x)-f(y)\|_{Y}}{\|x-y\|_{X}} .
$$

When $\Omega=\mathbb{R}^{n}$ we shall also use the shorter notation $\|f\|_{\text {Lip }}=\|f\|_{\operatorname{Lip}\left(\mathbb{R}^{n}\right)}$.

We shall use standard notation for vector-valued $L_{p}$ spaces. Specifically, for every measurable subset $\Omega \subseteq \mathbb{R}^{n}$ of positive Lebesgue measure and $p \in[1, \infty]$, we let $L_{p}(\Omega, Y)$ denote the space of all measurable functions $f: \Omega \rightarrow Y$ such that

$$
\|f\|_{L_{p}(\Omega, Y)} \stackrel{\operatorname{def}}{=}\left(\int_{\Omega}\|f(x)\|_{Y}^{p} \mathrm{~d} x\right)^{\frac{1}{p}}<\infty .
$$

Given $f: \Omega \rightarrow Y$ and $x \in \mathbb{R}^{n}$ we denote by $f^{x}: \Omega-x \rightarrow Y$ the translate of $f$ by $x$, i.e.,

$$
\forall y \in \Omega-x, \quad f^{x}(y) \stackrel{\text { def }}{=} f(x+y) .
$$

For $u \in(0, \infty)$ and $f \in L_{1}\left(u B^{n}, Y\right)$ let $T_{u} f: \mathbb{R}^{n} \rightarrow Y$ be the linear operator defined by

$$
\forall w \in \mathbb{R}^{n}, \quad T_{u} f(w) \stackrel{\text { def }}{=} \frac{n+2}{V_{n} u} \int_{B^{n}}\langle z, w\rangle f(u z) \mathrm{d} z,
$$

where

$$
V_{n} \stackrel{\text { def }}{=} \operatorname{vol}\left(B^{n}\right)=\frac{\pi^{n / 2}}{\Gamma\left(\frac{n}{2}+1\right)} .
$$

The operator norm of $T_{u} f$ can be bounded in terms of the Lipschitz constant of $f$ as follows.

Lemma 10. Fix $u \in(0, \infty)$ and a Lipschitz map $f: u B^{n} \rightarrow Y$. Then

$$
\left\|T_{u} f\right\|_{X \rightarrow Y} \leqslant\|f\|_{\operatorname{Lip}\left(u B^{n}\right)} .
$$

Proof. By rescaling we may assume that $u=1$. Take $w \in \mathbb{R}^{n} \backslash\{0\}$. For every $t \in \mathbb{R}$ consider the following affine hyperplane.

$$
H_{t} \stackrel{\text { def }}{=}\left\{y \in \mathbb{R}^{n}:\left\langle y, \frac{w}{\|w\|_{2}}\right\rangle=t\right\} \subseteq \mathbb{R}^{n} .
$$

Then by the definition (10) and Fubini's theorem we have

$$
\begin{aligned}
T_{1} f(w) & =\frac{(n+2)\|w\|_{2}}{V_{n}} \int_{-\infty}^{\infty} t \int_{H_{t} \cap B^{n}} f(z) \mathrm{d} z \mathrm{~d} t \\
& =\frac{(n+2)\|w\|_{2}}{V_{n}} \int_{0}^{\infty} t \int_{H_{t} \cap B^{n}}\left(f(z)-f\left(z-\frac{2 t w}{\|w\|_{2}}\right)\right) \mathrm{d} z \mathrm{~d} t
\end{aligned}
$$


Consequently,

$$
\begin{aligned}
\left\|T_{1} f(w)\right\|_{Y} & \leqslant \frac{(n+2)\|w\|_{2}}{V_{n}} \int_{0}^{\infty} t \int_{H_{t} \cap B^{n}}\left\|f(z)-f\left(z-\frac{2 t w}{\|w\|_{2}}\right)\right\|_{Y} \mathrm{~d} z \mathrm{~d} t \\
& \leqslant \frac{2(n+2)\|f\|_{\operatorname{Lip}\left(B^{n}\right)}\|w\|_{X}}{V_{n}} \int_{0}^{\infty} t^{2} \operatorname{vol}_{n-1}\left(H_{t} \cap B^{n}\right) \mathrm{d} t \\
& =\frac{(n+2)\|f\|_{\operatorname{Lip}\left(B^{n}\right)}\|w\|_{X}}{V_{n}} \int_{B^{n}} x_{1}^{2} \mathrm{~d} x \\
& =\|f\|_{\operatorname{Lip}\left(B^{n}\right)}\|w\|_{X} .
\end{aligned}
$$

We also record for future use the following simple estimate.

Lemma 11. For every $u \in(0, \infty)$ and $p \in[1, \infty]$ we have the following operator norm bound.

$$
\left\|T_{u}\right\|_{L_{p}\left(u B^{n}, Y\right) \rightarrow L_{p}\left(u B^{n}, Y\right)} \lesssim \min \{\sqrt{p n}, n\} .
$$

Proof. By rescaling we may assume that $u=1$. If $f \in L_{p}\left(B^{n}, Y\right)$ then

$$
\begin{aligned}
\left\|T_{1} f\right\|_{L_{p}\left(B^{n}, Y\right)} & \leqslant \frac{n+2}{V_{n}} \int_{B^{n}}\|w \mapsto\langle z, w\rangle f(z)\|_{L_{p}\left(B^{n}, Y\right)} \mathrm{d} z \\
& =\frac{n+2}{V_{n}}\left(\int_{B^{n}}\left|w_{1}\right|^{p} d w\right)^{\frac{1}{p}} \int_{B^{n}}\|z\|_{2} \cdot\|f(z)\|_{Y} \mathrm{~d} z \\
& \lesssim \min \{\sqrt{p n}, n\}\|f\|_{L_{p}\left(B^{n}, Y\right)},
\end{aligned}
$$

where in (11) we used the definition (10) and the triangle inequality in $L_{p}\left(B^{n}, Y\right)$, in (12) we used rotation invariance, and (13) follows from Hölder's inequality combined with the following fact, which can be verified by a direct computation and is also, say, a special case of inequality (4) in [5].

$$
\left(\frac{1}{V_{n}} \int_{B^{n}}\left|w_{1}\right|^{p} \mathrm{~d} w\right)^{\frac{1}{p}} \asymp \min \left\{\sqrt{\frac{p}{n}}, 1\right\} .
$$

For $u \in(0, \infty)$ and $f \in L_{1}\left(u B^{n}, Y\right)$ define

$$
P_{u}^{0} f \stackrel{\text { def }}{=} \frac{1}{V_{n}} \int_{B^{n}} f(u z) \mathrm{d} z \in Y .
$$

Thus, for every $\Omega \subseteq \mathbb{R}^{n}$ and $f \in L_{1}(\Omega, Y)$, if $x \in \mathbb{R}^{n}$ and $u \in(0, \infty)$ satisfy $x+u B^{n} \subseteq \Omega$ then the vector $P_{u}^{0} f^{x} \in Y$ is the mean of $f$ over $x+u B^{n}$. The following simple estimate will be used later.

Lemma 12. Fix $p \in[1, \infty), q \in(0, \infty)$ and $x \in \mathbb{R}^{n}$. Every measurable $f: \mathbb{R}^{n} \rightarrow Y$ satisfies

$$
\int_{0}^{\infty} \int_{B^{n}} \frac{\left\|f^{x}(u y)-P_{u}^{0} f^{x}\right\|_{Y}^{p}}{u^{q+1}} \mathrm{~d} y \mathrm{~d} u \leqslant \frac{2^{p}}{n+q} \int_{\mathbb{R}^{n}} \frac{\left\|f^{x}(y)-f(x)\right\|_{Y}^{p}}{\|y\|_{2}^{n+q}} \mathrm{~d} y .
$$


QuAntitative AfFine ApProximation for UMD TARgets

Proof. Recalling (14), it follows from the triangle inequality in $L_{p}\left(B^{n}, Y\right)$ and convexity that

$$
\forall v \in Y, \quad \int_{B^{n}}\left\|f^{x}(u y)-P_{u}^{0} f^{x}\right\|_{Y}^{p} \mathrm{~d} y \leqslant 2^{p} \int_{B^{n}}\left\|f^{x}(u y)-v\right\|_{Y}^{p} \mathrm{~d} y
$$

By choosing here $v=f(x)$, we see that for every $u \in(0, \infty)$ we have

$$
\int_{B^{n}}\left\|f^{x}(u y)-P_{u}^{0} f^{x}\right\|_{Y}^{p} \mathrm{~d} y \leqslant 2^{p} \int_{B^{n}}\left\|f^{x}(u y)-f(x)\right\|_{Y}^{p} \mathrm{~d} y .
$$

Hence, denoting the surface measure on $S^{n-1}$ by $\sigma$, by integrating in polar coordinates we get

$$
\begin{aligned}
\int_{0}^{\infty} \int_{B^{n}} \frac{\left\|f^{x}(u y)-P_{u}^{0} f^{x}\right\|_{Y}^{p}}{u^{q+1}} \mathrm{~d} y \mathrm{~d} u & \leqslant 2^{p} \int_{0}^{\infty} \int_{B^{n}} \frac{\left\|f^{x}(u y)-f(x)\right\|_{Y}^{p}}{u^{q+1}} \mathrm{~d} y \mathrm{~d} u \\
& =2^{p} \int_{0}^{\infty} \int_{0}^{1} \int_{S^{n-1}} r^{n-1} \frac{\left\|f^{x}(u r w)-f(x)\right\|_{Y}^{p}}{u^{q+1}} \mathrm{~d} \sigma(w) \mathrm{d} r \mathrm{~d} u \\
& =2^{p} \int_{0}^{1} \int_{0}^{\infty} \int_{S^{n-1}} r^{n-1} \frac{\left\|f^{x}(s w)-f(x)\right\|_{Y}^{p}}{(s / r)^{q+1}} \mathrm{~d} \sigma(w) \frac{\mathrm{d} s}{r} \mathrm{~d} r \\
& =\frac{2^{p}}{n+q} \int_{\mathbb{R}^{n}} \frac{\left\|f^{x}(y)-f(x)\right\|_{Y}^{p}}{\|y\|_{2}^{n+q}} \mathrm{~d} y .
\end{aligned}
$$

Define an affine mapping $P_{u}^{1} f: \mathbb{R}^{n} \rightarrow Y$ by

$$
P_{u}^{1} f \stackrel{\text { def }}{=} P_{u}^{0} f+T_{u} f
$$

By a simple change of variable, for every $y \in \mathbb{R}^{n}$ we have

$$
\left(P_{u}^{1} f^{x}\right)^{-x}(y)=P_{u}^{0} f^{x}+\sum_{j=1}^{n} \frac{y_{j}-x_{j}}{\int_{x+u B}\left(w_{j}-x_{j}\right)^{2} \mathrm{~d} w} \int_{x+u B}\left(z_{j}-x_{j}\right) f(z) \mathrm{d} z .
$$

Consequently, if $f$ were a real-valued function then $\left(P_{u}^{1} f^{x}\right)^{-x}$ would be the orthogonal projection in $L_{2}\left(x+u B^{n}\right)$ of $f$ onto the subspace consisting of all the affine mappings. Lemma 13 below shows that for every $p \in[1, \infty]$, if $f \in L_{p}\left(x+u B^{n}, Y\right)$ then the distance between $f$ and $\left(P_{u}^{1} f^{x}\right)^{-x}$ in $L_{p}\left(x+u B^{n}, Y\right)$ is controlled by the distance of $f$ to the subspace of $L_{p}\left(x+u B^{n}, Y\right)$ consisting of all the affine mappings. Such a statement was previously proved in [26], but since the dependence on $n$ and $p$ is important in the present context, and is only implicit in [26], we include its proof.

Lemma 13. Fix $p \in[1, \infty], u \in(0, \infty)$ and $x \in \mathbb{R}^{n}$. Suppose that $f \in L_{p}\left(x+u B^{n}, Y\right)$ and that $\Lambda: \mathbb{R}^{n} \rightarrow Y$ is affine. Then

$$
\left\|f^{x}-P_{u}^{1} f^{x}\right\|_{L_{p}\left(u B^{n}, Y\right)}=\left\|f-\left(P_{u}^{1} f^{x}\right)^{-x}\right\|_{L_{p}\left(x+u B^{n}, Y\right)} \lesssim \min \{\sqrt{p n}, n\} \cdot\|f-\Lambda\|_{L_{p}\left(x+u B^{n}, Y\right)} .
$$




\section{TuOmas Hytönen, SEAn Li, AND AsSaf NaOR}

Proof. By translation and a rescaling we may assume that $x=0$ and $u=1$. Since $P_{1}^{1} \Lambda=\Lambda$,

$$
\begin{aligned}
&\left\|f-P_{1}^{1} f\right\|_{L_{p}\left(B^{n}, Y\right)} \leqslant\|f-\Lambda\|_{L_{p}\left(B^{n}, Y\right)}+\left\|P_{1}^{1}(f-\Lambda)\right\|_{L_{p}\left(B^{n}, Y\right)} \\
& \stackrel{(14)}{\leqslant}\|f-\Lambda\|_{L_{p}\left(B^{n}, Y\right)}+\left\|P_{1}^{0}(f-\Lambda)\right\|_{L_{p}\left(B^{n}, Y\right)}+\left\|T_{1}(f-\Lambda)\right\|_{L_{p}\left(B^{n}, Y\right)} .
\end{aligned}
$$

It remains to note that $\left\|P_{1}^{0}(f-\Lambda)\right\|_{L_{p}\left(B^{n}, Y\right)} \leqslant\|f-\Lambda\|_{L_{p}\left(B^{n}, Y\right)}$ since $P_{1}^{0}$ is an averaging operator, and to apply Lemma 11 .

We end this section by recording for ease of later reference two consequences of Lemma 13.

Corollary 14. Fix $p \in[1, \infty), q \in(0, \infty)$ and $x \in \mathbb{R}^{n}$. Every measurable $f: \mathbb{R}^{n} \rightarrow Y$ satisfies

$$
\left(\int_{0}^{\infty} \int_{B^{n}} \frac{\left\|f^{x}(u y)-P_{u}^{1} f^{x}(u y)\right\|_{Y}^{p}}{u^{q+1}} \mathrm{~d} y \mathrm{~d} u\right)^{\frac{1}{p}} \lesssim \frac{\min \{\sqrt{p n}, n\}}{(q+n)^{\frac{1}{p}}}\left(\int_{\mathbb{R}^{n}} \frac{\left\|f^{x}(y)-f(x)\right\|_{Y}^{p}}{\|y\|_{2}^{n+q}} \mathrm{~d} y\right)^{\frac{1}{p}} .
$$

Proof. Fix $u \in(0, \infty)$. Lemma 13 implies that every affine mapping $\Lambda: \mathbb{R}^{n} \rightarrow Y$ satisfies

$$
\left(\int_{B^{n}}\left\|f^{x}(u y)-P_{u}^{1} f^{x}(u y)\right\|_{Y}^{p} \mathrm{~d} y\right)^{\frac{1}{p}} \lesssim \min \{\sqrt{p n}, n\}\left(\int_{B^{n}}\left\|f^{x}(u y)-\Lambda(u y)\right\|_{Y}^{p} \mathrm{~d} y\right)^{\frac{1}{p}} .
$$

An application of (17) when $\Lambda$ is the constant $P_{u}^{0} f^{x}$ shows that for every $u \in(0, \infty)$ we have

$$
\left(\int_{B^{n}}\left\|f^{x}(u y)-P_{u}^{1} f^{x}(u y)\right\|_{Y}^{p} \mathrm{~d} y\right)^{\frac{1}{p}} \lesssim \min \{\sqrt{p n}, n\}\left(\int_{B^{n}}\left\|f^{x}(u y)-P_{u}^{0} f^{x}\right\|_{Y}^{p} \mathrm{~d} y\right)^{\frac{1}{p}} .
$$

This implies the desired estimate due to Lemma 12.

Corollary 15. Fix $p \in[1, \infty), q \in(p, \infty)$ and $x \in \mathbb{R}^{n}$. Suppose that $f: \mathbb{R}^{n} \rightarrow Y$ is smooth. Then

$$
\begin{aligned}
& \left(\int_{0}^{\infty} \int_{B^{n}} \frac{\left\|f^{x}(u y)-P_{u}^{1} f^{x}(u y)\right\|_{Y}^{p}}{u^{q+1}} \mathrm{~d} y \mathrm{~d} u\right)^{\frac{1}{p}} \\
& \qquad \frac{p \min \{\sqrt{p n}, n\}}{q(n+q-p)^{\frac{1}{p}}} \sum_{j=1}^{n}\left(\int_{\mathbb{R}^{n}}\left\|\frac{\partial f}{\partial x_{j}}(x+y)-\frac{\partial f}{\partial x_{j}}(x)\right\|_{Y}^{p} \frac{\mathrm{d} y}{\|y\|_{2}^{n+q-p}}\right)^{\frac{1}{p}} .
\end{aligned}
$$

Proof. Suppose that $x \in \mathbb{R}^{n}$ and $u \in(0, \infty)$. For every $j \in\{1, \ldots, n\}$ define

$$
a_{j}(u, x) \stackrel{\text { def }}{=} \int_{0}^{1} P_{s u}^{0} f_{j}^{x} \mathrm{~d} s \in Y, \quad \text { where } \quad f_{j} \stackrel{\text { def }}{=} \frac{\partial f}{\partial x_{j}} .
$$

Also, define an affine function $\Lambda_{x, u}: \mathbb{R}^{n} \rightarrow Y$ by setting

$$
\forall y \in \mathbb{R}^{n}, \quad \Lambda_{x, u}(y) \stackrel{\text { def }}{=} f(x)+\sum_{j=1}^{n} y_{j} a_{j}(u, x) .
$$


An application of (17) with $\Lambda=\Lambda_{x, u}$ shows that

$$
\begin{aligned}
\left(\int_{0}^{\infty} \int_{B^{n}} \frac{\left\|f^{x}(u y)-P_{u}^{1} f^{x}(u y)\right\|_{Y}^{p}}{u^{q+1}} \mathrm{~d} y \mathrm{~d} u\right)^{\frac{1}{p}} & \\
& \lesssim \min \{\sqrt{p n}, n\}\left(\int_{0}^{\infty} \int_{B^{n}} \frac{\left\|f^{x}(u y)-\Lambda_{x, u}(u y)\right\|_{Y}^{p}}{u^{q+1}} \mathrm{~d} y \mathrm{~d} u\right)^{\frac{1}{p}} .
\end{aligned}
$$

Observe that

$$
f^{x}(u y)-\Lambda_{x, u}(u y) \stackrel{(20)}{=} \int_{0}^{1} \frac{d}{d s} f^{x}(s u y) \mathrm{d} s-\sum_{j=1}^{n} u y_{j} a_{j}(u, x) \stackrel{(19)}{=} \sum_{j=1}^{n} \int_{0}^{1} u y_{j}\left(f_{j}^{x}(s u y)-P_{s u}^{0} f_{j}^{x}\right) \mathrm{d} s .
$$

By the triangle inequality in $L_{p}\left(B^{n}, Y\right)$, this implies that for every $u \in(0, \infty)$,

$$
\begin{aligned}
\left(\int_{B^{n}}\left\|f^{x}(u y)-\Lambda_{x, u}(u y)\right\|_{Y}^{p} \mathrm{~d} y\right)^{\frac{1}{p}} & \leqslant \sum_{j=1}^{n} \int_{0}^{1} u\left(\int_{B^{n}}\left|y_{j}\right|^{p}\left\|f_{j}^{x}(s u y)-P_{s u}^{0} f_{j}^{x}\right\|_{Y}^{p} \mathrm{~d} y\right)^{\frac{1}{p}} \mathrm{~d} s \\
& \leqslant \sum_{j=1}^{n} \int_{0}^{u}\left(\int_{B^{n}}\left\|f_{j}^{x}(t y)-P_{t}^{0} f_{j}^{x}\right\|_{Y}^{p} \mathrm{~d} y\right)^{\frac{1}{p}} \mathrm{~d} t=\sum_{j=1}^{n} \int_{0}^{u} h_{j}(t) \mathrm{d} t
\end{aligned}
$$

where we used the crude estimate $\max _{y \in B^{n}} \max _{j \in\{1, \ldots, n\}}\left|y_{j}\right| \leqslant 1$ and introduced the notation

$$
\forall(j, t) \in\{1, \ldots, n\} \times(0, \infty), \quad h_{j}(t) \stackrel{\text { def }}{=}\left(\int_{B^{n}}\left\|f_{j}^{x}(t y)-P_{t}^{0} f_{j}^{x}\right\|_{Y}^{p} \mathrm{~d} y\right)^{\frac{1}{p}} .
$$

A combination of (22) with the triangle inequality and Hardy's inequality [72, Section A.4] yields

$$
\begin{gathered}
\left(\int_{0}^{\infty} \int_{B^{n}} \frac{\left\|f^{x}(u y)-\Lambda_{x, u}(u y)\right\|_{Y}^{p}}{u^{q+1}} \mathrm{~d} y \mathrm{~d} u\right)^{\frac{1}{p}} \leqslant \sum_{j=1}^{n}\left(\int_{0}^{\infty}\left(\int_{0}^{u} h_{j}(t) \mathrm{d} t\right)^{p} \frac{\mathrm{d} u}{u^{q+1}}\right)^{\frac{1}{p}} \\
\leqslant \sum_{j=1}^{n} \frac{p}{q}\left(\int_{0}^{\infty} \frac{h_{j}(u)^{p}}{u^{q-p+1}} \mathrm{~d} u\right)^{\frac{1}{p}} \stackrel{(23)}{=} \frac{p}{q} \sum_{j=1}^{n}\left(\int_{0}^{\infty} \int_{B^{n}}\left\|f_{j}^{x}(t y)-P_{t}^{0} f_{j}^{x}\right\|_{Y}^{p} \frac{\mathrm{d} u}{u^{q-p+1}}\right)^{\frac{1}{p}} .
\end{gathered}
$$

By applying Lemma 12 to each of the functions $\left\{h_{j}\right\}_{j=1}^{n}$ (with $q$ replaced by $q-p$ ), we conclude from (24) that the following estimate holds true.

$$
\left(\int_{0}^{\infty} \int_{B^{n}} \frac{\left\|f^{x}(u y)-\Lambda_{x, u}(u y)\right\|_{Y}^{p}}{u^{q+1}} \mathrm{~d} y \mathrm{~d} u\right)^{\frac{1}{p}} \lesssim \frac{p}{q(n+q-p)^{\frac{1}{p}}} \sum_{j=1}^{n}\left(\int_{\mathbb{R}^{n}} \frac{\left\|f_{j}^{x}(y)-f_{j}(x)\right\|_{Y}^{p}}{\|y\|_{2}^{n+q-p}} \mathrm{~d} y\right)^{\frac{1}{p}} .
$$

Recalling the definition of $f_{j}$ in (19), the desired estimate (18) now follows from (21). 


\subsection{Proof of Lemma 4}

Fix $\varepsilon \in(0,1)$ and denote $\delta \stackrel{\text { def }}{=}(\varepsilon / 9)^{1+n / p}$. Fix also $0<r<r_{p}^{X \rightarrow Y}(\delta)$ and a 1-Lipschitz function $f: B_{X} \rightarrow$ $Y$. By the definition of $r_{p}^{X \rightarrow Y}(\delta)$, we can find $y \in X$ and $\rho \geqslant r$ such that $y+\rho B_{X} \subseteq B_{X}$, and there exists an affine mapping $\Lambda: X \rightarrow Y$ with $\|\Lambda\|_{\text {Lip }} \leqslant 3$, such that

$$
\frac{1}{\operatorname{vol}\left(\rho B_{X}\right)} \int_{y+\rho B_{X}}\|f(u)-\Lambda(u)\|_{Y}^{p} \mathrm{~d} u \leqslant \delta^{p} \rho^{p}=\frac{\varepsilon^{p+n} \rho^{p}}{9^{p+n}} .
$$

We claim that (25), combined with the fact that $\|f-\Lambda\|_{\text {Lip }} \leqslant\|f\|_{\text {Lip }}+\|\Lambda\|_{\text {Lip }} \leqslant 4$, yields

$$
\forall z \in y+\rho B_{X}, \quad\|f(z)-\Lambda(z)\|_{Y} \leqslant \varepsilon \rho,
$$

thus completing the proof of Lemma 4.

Assume for the sake of obtaining a contradiction that (26) fails. Then there exists $z \in y+\rho B_{x}$ such that $\|f(z)-\Lambda(z)\|_{Y}>\varepsilon \rho$. Write $\lambda=\varepsilon / 9$ and define $w=\lambda y+(1-\lambda) z$. Observe that we have $\|w-z\|_{X}=\lambda\|y-z\|_{X} \leqslant \lambda \rho$. Supposing that $u \in X$ satisfies $\|u-w\|_{X} \leqslant \lambda \rho$, we therefore obtain

$$
\|u-y\|_{X}=\|(u-w)-(1-\lambda)(y-z)\|_{X} \leqslant\|u-w\|+(1-\lambda)\|y-z\|_{X} \leqslant \rho .
$$

Moreover, since $\|f-\Lambda\|_{\text {Lip }} \leqslant 4$ we have

$$
\begin{aligned}
\|f(u)-\Lambda(u)\|_{Y} \geqslant\|f(z)-\Lambda(z)\|_{Y}-4\|u-z\|_{X} \\
\quad>\varepsilon \rho-4\|u-w\|_{X}-4\|w-z\|_{X} \geqslant \varepsilon \rho-8 \lambda \rho=\lambda \rho .
\end{aligned}
$$

It follows from (27) that $w+\lambda \rho B_{X} \subseteq y+\rho B_{X}$, and it follows from (28) that for $u \in w+\lambda \rho B_{X}$ the integrand in the left hand side of (25) is strictly larger than $\lambda \rho$. Hence, (25) yields the following contradiction.

$$
\lambda^{p+n} \rho^{p}=\frac{\varepsilon^{p+n} \rho^{p}}{9^{p+n}}>\frac{\operatorname{vol}\left(\lambda \rho B_{X}\right)}{\operatorname{vol}\left(\rho B_{X}\right)} \lambda^{p} \rho^{p}=\lambda^{p+n} \rho^{p} .
$$

\subsection{An upper bound on the modulus of affine approximability}

The example that is constructed below was obtained in collaboration with Charles Fefferman; we thank him for agreeing that we include it here. A simple construction from [57] shows that if $p \in[2, \infty)$ and $\varepsilon \in(0,1)$ then

$$
r^{\ell_{2}^{n} \rightarrow \ell_{2}\left(\ell_{p}\right)}(\varepsilon) \lesssim \frac{e^{-1 /(c \varepsilon)^{p}}}{\sqrt{n}}
$$

where $c \in(0, \infty)$ is a universal constant. We shall now show how the example of [57] can be tensorized so as to yield an improved dependence on $n$, and we shall also briefly discuss the problem of bounding $r_{q}^{X \rightarrow Y}(\varepsilon)$ for finite $q \geqslant 1$. The following lemma is an $L_{q}$-variant of Lemma 4.1 of [57]. 
Lemma 16. There exists a universal constant $C \in(0, \infty)$ with the following property. For every $\varepsilon \in(0,1)$ and $p \in[1, \infty)$ there exists a 1 -Lipschitz function $f: \mathbb{R} \rightarrow \ell_{p}$ such that for every $q \in[1, \infty]$ and every affine mapping $\Lambda: \mathbb{R} \rightarrow \ell_{p}$, if $a, b \in \mathbb{R}$ satisfy $-1 \leqslant a<b \leqslant 1$ then

$$
\frac{b-a}{2} \geqslant 4 e^{-(C / \varepsilon)^{p}} \Longrightarrow\left(\frac{1}{b-a} \int_{a}^{b}\|f(x)-\Lambda(x)\|_{p}^{q} \mathrm{~d} x\right)^{\frac{1}{q}}>\varepsilon \cdot \frac{b-a}{2} .
$$

Consequently,

$$
r_{q}^{\mathbb{R} \rightarrow \ell_{p}}(\varepsilon) \lesssim e^{-(C / \varepsilon)^{p}}
$$

Proof. Let $\varphi: \mathbb{R} \rightarrow \mathbb{R}$ be the piecewise affine ("sawtooth") function defined by $\varphi(2 \mathbb{Z})=\{0\}$ and $\varphi(1+2 \mathbb{Z})=\{1\}$. Fix $m \in \mathbb{N}$ that will be determined later. Denoting the standard basis of $\ell_{p}^{m}$ by $\left\{e_{j}\right\}_{j=1}^{m}$, define $f: \mathbb{R} \rightarrow \ell_{p}^{m}$ by setting for every $x \in \mathbb{R}$,

$$
f(x) \stackrel{\text { def }}{=} \frac{1}{m^{1 / p}} \sum_{k=1}^{m} \frac{\varphi\left(2^{k} x\right)}{2^{k}} e_{k} .
$$

Since $\varphi$ is 1-Lipschitz, it follows from (31) that also $f$ is 1-Lipschitz.

Fix $a, b \in \mathbb{R}$ satisfying $-1 \leqslant a \leqslant b \leqslant 1$ and $b-a \geqslant 4 / 2^{m}$. There exists $k \in\{1, \ldots, m\}$ such that $4 / 2^{k} \leqslant b-a \leqslant 8 / 2^{k}$. Hence there is $j \in\left\{0, \ldots, 2^{k-1}-1\right\}$ for which $\left[j / 2^{k-1},(j+1) / 2^{k-1}\right] \subseteq[a, b]$. Then, since $b-a \leqslant 8 / 2^{k}$, for every affine mapping $\Lambda: \mathbb{R} \rightarrow \ell_{p}^{m}$ we have

$$
\frac{1}{b-a} \int_{a}^{b}\|f(x)-\Lambda(x)\|_{p}^{q} \mathrm{~d} x \gtrsim 2^{k} \int_{j / 2^{k-1}}^{(j+1) / 2^{k-1}}\|f(x)-\Lambda(x)\|_{p}^{q} \mathrm{~d} x .
$$

Writing $\Lambda=\left(\Lambda_{1}, \ldots, \Lambda_{m}\right)$, where $\Lambda_{1}, \ldots, \Lambda_{m}: \mathbb{R} \rightarrow \mathbb{R}$ are affine, it follows from (31) and (32) that

$$
\begin{aligned}
\frac{1}{b-a} \int_{a}^{b}\|f(x)-\Lambda(x)\|_{p}^{q} \mathrm{~d} x & \gtrsim 2^{k} \int_{j / 2^{k-1}}^{(j+1) / 2^{k-1}}\left|\frac{\varphi\left(2^{k} x\right)}{m^{1 / q 2^{k}}}-\Lambda_{k}(x)\right|^{q} \mathrm{~d} x \\
& =\frac{1}{m^{q / p} 2^{q k}} \int_{-1}^{1}|\varphi(y)-\mathscr{L}(y)|^{q} \mathrm{~d} y,
\end{aligned}
$$

where $\mathscr{L}: \mathbb{R} \rightarrow \mathbb{R}$ is the affine function given for every $y \in \mathbb{R}$ by $\mathscr{L}(y) \stackrel{\text { def }}{=} m^{1 / p} 2^{k} \Lambda_{k}\left((y+2 j+1) / 2^{k}\right)$. Recalling (14) and (16), we have $P_{1}^{1} \varphi \equiv 1 / 2$. Hence, by Lemma 13,

$$
\left(\int_{-1}^{1}|\varphi(y)-\mathscr{L}(y)|^{q} \mathrm{~d} y\right)^{\frac{1}{q}} \gtrsim\left(\int_{-1}^{1}\left|\varphi(y)-\frac{1}{2}\right|^{q} \mathrm{~d} y\right)^{\frac{1}{q}} \gtrsim 1 .
$$

Since $b-a \asymp 2^{-k}$, by combining (33) and (34) we see that

$$
\left(\frac{1}{b-a} \int_{a}^{b}\|f(x)-\Lambda(x)\|_{p}^{q} \mathrm{~d} x\right)^{\frac{1}{q}} \geqslant \frac{\eta}{m^{1 / p}} \cdot \frac{b-a}{2},
$$




\section{TUOMAS Hytönen, SEAN LI, AND ASSAF NAOR}

where $\eta \in(0, \infty)$ is a universal constant. Suppose that $\varepsilon<\eta$ and choose $m$ to be the largest positive integer such that $m<(\eta / \varepsilon)^{p}$. Then (35) implies the conclusion of (30). The requirement here is that $b-a \geqslant 4 / 2^{m}$, and since $m+1 \geqslant(\eta / \varepsilon)^{p}$, this requirement is satisfied if $b-a \geqslant 8 / 2^{(\eta / \varepsilon)^{p}}$, which follows from $(b-a) / 2 \geqslant 4 e^{-(\eta /(2 \varepsilon))^{p}}$. Thus (30) holds true with $C=\eta / 2$. Note that, with this choice of $C$, the implication (30) holds vacuously when $\varepsilon \geqslant \eta$.

Lemma 17 below tensorizes Lemma 16 to improve over (29), obtaining exponential decay.

Lemma 17. There exists universal constants $K, \varepsilon_{0} \in(0,1)$ such that for every $\varepsilon \in\left(0, \varepsilon_{0}\right]$, every $p \in[1, \infty)$ and every $n \in \mathbb{N}$ we have

$$
r^{\ell^{n} \rightarrow \ell_{2}\left(\ell_{p}\right)}(\varepsilon) \leqslant e^{-n(K / \varepsilon)^{p}} .
$$

Proof. By Lemma 16 (with $q=\infty$ ) we can fix $K, \varepsilon_{0} \in(0,1)$ such that for every $\varepsilon \in\left(0, \varepsilon_{0}\right]$ and $p \in$ $[1, \infty)$ there exists a 1-Lipschitz mapping $f:[-1,1] \rightarrow \ell_{p}$ such that for every interval $[a, b] \subseteq[-1,1]$ with $(b-a) / 2>e^{-(K / \varepsilon)^{p}}$ and for every affine mapping $\Lambda: \mathbb{R} \rightarrow \ell_{p}$ there exists $x \in[a, b]$ such that $\|f(x)-\Lambda(x)\|_{p}>\varepsilon(b-a) / 2$.

Define $F: \mathbb{R}^{n} \rightarrow \ell_{2}^{n}\left(\ell_{p}\right)$ by setting for every $x=\left(x_{1}, \ldots, x_{n}\right) \in \mathbb{R}^{n}$,

$$
F(x) \stackrel{\text { def }}{=}\left(f\left(x_{1}\right), \frac{f\left(e^{(K / \varepsilon)^{p}} x_{2}\right)}{e^{(K / \varepsilon)^{p}}}, \ldots, \frac{f\left(e^{(n-1)(K / \varepsilon)^{p}} x_{n}\right)}{e^{(n-1)(K / \varepsilon)^{p}}}\right) \in \ell_{2}^{n}\left(\ell_{p}\right) .
$$

By applying the 1-Lipschitz condition for $f$ coordinate-wise, we see that $F$ is 1-Lipschitz as a mapping from $\ell_{2}^{n}$ to $\ell_{2}^{n}\left(\ell_{p}\right)$. Fix $x \in B^{n}$ and $r \in(0,1)$ such that $x+r B^{n} \subseteq B^{n}$. Suppose from now on that $r>e^{-n(K / \varepsilon)^{p}}$ and let $j \in \mathbb{N}$ be the largest integer for which $r \leqslant e^{-(j-1)(\bar{K} / \varepsilon)^{p}}$. Then $j \in\{1, \ldots, n\}$ and $e^{(j-1)(K / \varepsilon)^{p}} r>$ $e^{-(K / \varepsilon)^{p}}$. Let $\Lambda: \mathbb{R}^{n} \rightarrow \ell_{2}^{n}\left(\ell_{p}\right)$ be an affine mapping, and write $\Lambda=\left(\Lambda_{1}, \ldots, \Lambda_{n}\right)$, where $\Lambda_{1}, \ldots, \Lambda_{n}: \mathbb{R}^{n} \rightarrow$ $\ell_{p}$ are affine. Consider the affine mapping $\Lambda_{j}^{\prime}: \mathbb{R} \rightarrow \ell_{p}$ given by $\Lambda_{j}^{\prime}(y)=\Lambda_{j}\left(x_{1}, \ldots, x_{j-1}, y, x_{j+1}, \ldots, x_{n}\right)$. Set $a=x_{j} e^{(j-1)(K / \varepsilon)^{p}}-r e^{(j-1)(K / \varepsilon)^{p}}$ and $b=x_{j} e^{(j-1)(K / \varepsilon)^{p}}+r e^{(j-1)(K / \varepsilon)^{p}}$. Then

$$
\frac{b-a}{2}=r e^{(j-1)(K / \varepsilon)^{p}}>e^{-(K / \varepsilon)^{p}}
$$

so by our assumption on $f$ there exists $w \in\left[x_{j} e^{(j-1)(K / \varepsilon)^{p}}-r e^{(j-1)(K / \varepsilon)^{p}}, x_{j} e^{(j-1)(K / \varepsilon)^{p}}+r e^{(j-1)(K / \varepsilon)^{p}}\right]$ with

$$
\left\|f(w)-e^{(j-1)(K / \varepsilon)^{p}} \Lambda_{j}^{\prime}\left(w e^{-(j-1)(K / \varepsilon)^{p}}\right)\right\|_{p}>\varepsilon r e^{(j-1)(K / \varepsilon)^{p}} .
$$

Setting $y=w e^{-(j-1)(K / \varepsilon)^{p}}$, this is the same as asserting that there exists $y \in\left[x_{j}-r, x_{j}+r\right]$ with

$$
\left\|e^{-(j-1)(K / \varepsilon)^{p}} f\left(e^{(j-1)(K / \varepsilon)^{p}} y\right)-\Lambda_{j}^{\prime}(y)\right\|_{p}>\varepsilon r .
$$

Hence, writing $z=\left(x_{1}, \ldots, x_{j-1}, y, x_{j+1}, \ldots, x_{n}\right)$, we have that $z \in x+r B^{n}$ and

$$
\|F(z)-\Lambda(z)\|_{\ell_{2}^{n}\left(\ell_{p}\right)} \geqslant\left\|e^{-(j-1)(K / \varepsilon)^{p}} f\left(e^{(j-1)(K / \varepsilon)^{p}} y\right)-\Lambda_{j}^{\prime}(y)\right\|_{p}>\varepsilon r
$$

Since (37) holds true for every affine mapping $\Lambda: \mathbb{R}^{n} \rightarrow \ell_{2}^{n}\left(\ell_{p}\right)$ whenever $x+r B^{n} \subseteq B^{n}$ and $r>e^{-n(K / \varepsilon)^{p}}$, the proof of (36) is complete. 
Remark 18. An upper bound on $r_{p}^{\ell_{2}^{n} \rightarrow \ell_{2}\left(\ell_{p}\right)}(\varepsilon)$ is a consequence of Lemma 17 and Lemma 4. It seems likely that a significantly stronger upper bound holds true, but the above tensorization procedure does not seem to yield such an improvement. We leave the investigation of upper bounds on the modulus of $L_{p}$ affine approximability as an interesting question for future research.

\section{A UMD-valued Dorronsoro-type estimate}

The main ingredient of the proof of Theorem 5 (hence also Theorem 2) is the following result.

Theorem 19. There exists a universal constant $\kappa \in[2, \infty)$ with the following property. Suppose that $\left(Y,\|\cdot\|_{Y}\right)$ is a UMD Banach space and write $\beta=\beta(Y)$. Suppose that $f: \mathbb{R}^{n} \rightarrow Y$ is a Lipschitz and compactly supported function. Then

$$
\left(\frac{1}{V_{n}} \iiint_{\mathbb{R}^{n} \times B^{n} \times(0, \infty)} \frac{\left\|f^{x}(u y)-P_{u}^{1} f^{x}(u y)\right\|_{Y}^{\kappa \beta}}{u^{1+\kappa \beta}} \mathrm{d} x \mathrm{~d} y \mathrm{~d} u\right)^{\frac{1}{\kappa \beta}} \lesssim \beta^{15} n^{\frac{5}{2}}(\operatorname{vol}(\operatorname{supp}(f)))^{\frac{1}{\kappa \beta}}\|f\|_{\text {Lip }} .
$$

In the case of real-valued functions on $\mathbb{R}^{n}$, such a statement was first proved by Dorronsoro [26], with an implicit dependence on the dimension $n$. Extensions and variants of Dorronsoro's theorem have been further studied within the theory of functions spaces, where norms like the one that appears on the left of (38) define what is called local approximation spaces. See [75, Section 1.7] for some discussion of the subsequent history and [75, Section 3.5.1] for a different proof of a similar statement. There, the dependence on $n$ is perhaps even more implicit, since it also depends on a non-canonical choice of resolutions of identity used to define some general function spaces.

This dependence on dimension is crucial for us here; specifically, we desire polynomial growth in $n$ in the right hand side of (38), while a naïve examination of the proof in [26] reveals that it yields a much worse (super-exponential) dependence on $n$. Note also that in [26] the $L_{\kappa \beta}$ norm that appears in (38) can be replaced by an $L_{p}$ norm for any $p \in(1, \infty)$, while in the present vector-valued setting the geometry of the target space $Y$ influences the value of $p$. In fact, we shall prove a more refined (and stronger) version of Theorem 19; see Theorem 40 below, in which the $L_{\kappa \beta}$ norm that appears in (38) can be replaced by an $L_{p}$ norm provided $Y$ is a UMD Banach space of Rademacher cotype $p$ (see Section 4.4 below). These refinements are not important for our purposes, i.e., for proving Theorem 5, but they do imply sharper results, e.g. when $Y$ is itself an $L_{q}(\mu)$ space. In the same vein, the exponent 15 of $\beta$ in (38) is not sharp (for the purpose of Theorem 5 we only desire a polynomial dependence on $\beta$ ).

The bulk of the ensuing discussion is devoted to the proof of Theorem 19. Our argument roughly follows the strategy of Dorronsoro in [26], combined with substantial additions and modifications in order to obtain good dependence on $n$ and also overcome difficulties that arise in the vector-valued setting and are not present in the real-valued setting of [26]. As explained in the Introduction, these complications reflect a genuine difference between the vector-valued setting and the real-valued setting, as such results do not hold true for general Banach space targets $Y$, so the geometry of $Y$ must somehow enter into the argument. We did not investigate the extent to which Theorem 19 (and Theorem 40 below) are sharp in terms of the assumptions that are required from $Y$ and the $L_{p}$ norm that could appear in (38) (or (137) below).

DisCRETE ANALYsis, 2016:6, 48pp. 


\section{TuOmas Hytönen, SEAn Li, AND AsSaf NaOR}

\subsection{Proof of Theorem 5 assuming the validity of Theorem 19}

Here we shall prove Theorem 5 while using Theorem 19. This will allow us to focus later on Theorem 19 itself in order complete the justification of Theorem 5, and hence, by Lemma 4, also (3) and Theorem 2.

Recalling our setting, we are given a $n$-dimensional normed space $\left(X=\mathbb{R}^{n},\|\cdot\|_{X}\right)$ such that (8) holds true. We are also given a normed space $\left(Y,\|\cdot\|_{Y}\right)$ that satisfies the assumptions of Theorem 19. Suppose that $f: B_{X} \rightarrow Y$ is 1-Lipschitz. Without loss of generality assume also that $f(0)=0$.

Define $\phi:[0, \infty) \rightarrow[0, \infty)$ by

$$
\forall u \in[0, \infty), \quad \phi(u) \stackrel{\text { def }}{=} \begin{cases}1 & \text { if } u \leqslant \frac{1}{\sqrt{n}}, \\ n+1-n^{\frac{3}{2}} u & \text { if } \frac{1}{\sqrt{n}}<u \leqslant\left(1+\frac{1}{n}\right) \frac{1}{\sqrt{n}}, \\ 0 & \text { if } u>\left(1+\frac{1}{n}\right) \frac{1}{\sqrt{n}} .\end{cases}
$$

Then $\phi$ is supported in $[0,(1+1 / n) / \sqrt{n}]$ and it is elementary to verify the following inequalities.

$$
\forall u \in(0, \infty), \quad \max \{\phi(u), \sqrt{n} u \phi(u)\} \leqslant 1 .
$$

and

$$
\forall u, v \in(0, \infty), \quad|\phi(u)-\phi(v)| \cdot \min \{u, v\} \leqslant(n+1)|u-v| .
$$

Since by (8) we have $\frac{1}{\sqrt{n}} B^{n} \subseteq B_{X}$, by (39) we know that every $x \in \mathbb{R}^{n}$ satisfies $\phi\left(\|x\|_{2}\right) x \in B_{X}$. We can therefore define $F: \mathbb{R}^{n} \rightarrow Y$ by $F(x)=f\left(\phi\left(\|x\|_{2}\right) x\right)$. Then, $F(x)=f(x)$ if $\|x\|_{2} \leqslant 1 / \sqrt{n}$, and $F(x)=0$ if $\|x\|_{2} \geqslant(1+1 / n) / \sqrt{n}$. Also, every $x, y \in \mathbb{R}^{n}$ with $\|y\|_{X} \leqslant\|x\|_{X}$ satisfy

$$
\begin{array}{rll}
\|F(x)-F(y)\|_{Y} & \leqslant\|f\|_{\operatorname{Lip}\left(B_{X}\right)}\left\|\phi\left(\|x\|_{2}\right) x-\phi\left(\|y\|_{2}\right) y\right\|_{X} \\
& \stackrel{\phi}{\leqslant}\left(\|x\|_{2}\right)\|x-y\|_{X}+\left|\phi\left(\|x\|_{2}\right)-\phi\left(\|y\|_{2}\right)\right|\|y\|_{X} \\
\stackrel{(8) \wedge(39)}{\leqslant} & \|x-y\|_{X}+\left|\phi\left(\|x\|_{2}\right)-\left\|\phi\left(\|y\|_{2}\right) \mid \sqrt{n}\right\| y \|_{2}\right. \\
& \stackrel{(40)}{\leqslant} & \|x-y\|_{X}+\sqrt{n}(n+1)\|x-y\|_{2} \\
& \stackrel{(8)}{\leqslant} & \left(n^{\frac{3}{2}}+\sqrt{n}+1\right)\|x-y\|_{X} .
\end{array}
$$

Thus $\|F\|_{\operatorname{Lip}\left(\mathbb{R}^{n}\right)} \lesssim n^{\frac{3}{2}}$. Since $\operatorname{supp}(F) \subseteq\left(1+\frac{1}{n}\right) \frac{1}{\sqrt{n}} B^{n}$, it therefore follows from Theorem 19 that

$$
\begin{aligned}
\left(\iiint_{\mathbb{R}^{n} \times B^{n} \times(0, \infty)} \frac{\left\|F^{x}(u y)-P_{u}^{1} F^{x}(u y)\right\|_{Y}^{\kappa \beta}}{\left.V_{n} u^{1+\kappa \beta} \mathrm{d} x \mathrm{~d} y \mathrm{~d} u\right)^{\frac{1}{\kappa \beta}}}\right. & \leqslant K \beta^{15} n^{\frac{5}{2}}\left(1+\frac{1}{n}\right)^{\frac{n}{\kappa \beta}}\left(\frac{V_{n}}{n^{\frac{n}{2}}}\right)^{\frac{1}{\kappa \beta}} n^{\frac{3}{2}} \\
& <e K \beta^{15} n^{4}\left(\frac{V_{n}}{n^{\frac{n}{2}}}\right)^{\frac{1}{\kappa \beta}},
\end{aligned}
$$

where $K \in(0, \infty)$ is a universal constant. Note that

$$
(x, u) \in\left(\left(1-\frac{1}{n}\right) \frac{1}{\sqrt{n}} B^{n}\right) \times\left(0, \frac{1}{n^{3 / 2}}\right) \Longrightarrow x+u B^{n} \subseteq \frac{1}{\sqrt{n}} B^{n} .
$$


Since $F$ coincides with $f$ on $\frac{1}{\sqrt{n}} B^{n}$, it follows that

$$
(x, y, u) \in\left(\left(1-\frac{1}{n}\right) \frac{1}{\sqrt{n}} B^{n}\right) \times B^{n} \times\left(0, \frac{1}{n^{3 / 2}}\right) \Longrightarrow P_{u}^{1} F^{x}=P_{u}^{1} f^{x} \quad \text { and } \quad F^{x}(u y)=f^{x}(u y) .
$$

These observations in combination with (41) imply that

$$
\left(\iiint_{\left(\left(1-\frac{1}{n}\right) \frac{1}{\sqrt{n}} B^{n}\right) \times B^{n} \times\left(0, \frac{1}{n^{3 / 2}}\right)} \frac{\left\|f^{x}(u y)-P_{u}^{1} f^{x}(u y)\right\|_{Y}^{\kappa \beta}}{V_{n} u^{1+\kappa \beta}} \mathrm{d} x \mathrm{~d} y \mathrm{~d} u\right)^{\frac{1}{\kappa \beta}}<K \beta^{15} n^{4}\left(\frac{V_{n}}{n^{\frac{n}{2}}}\right)^{\frac{1}{\kappa \beta}} .
$$

Fix $M \in(1, \infty)$ whose precise value will be specified later. It follows from (42) that there exist

$$
u \in\left(\frac{1}{M n^{2 / 3}}, \frac{1}{n^{3 / 2}}\right) \quad \text { and } \quad x \in\left(1-\frac{1}{n}\right) \frac{1}{\sqrt{n}} B^{n},
$$

such that

$$
\left(\frac{1}{V_{n}} \int_{B^{n}}\left\|f^{x}(u y)-P_{u}^{1} f^{x}(u y)\right\|_{Y}^{\kappa \beta} \mathrm{d} y\right)^{\frac{1}{\kappa \beta}} \leqslant \frac{2 e K \beta^{15} n^{4} u}{(\log M)^{\frac{1}{\kappa \beta}}} .
$$

Indeed, we would otherwise have

$$
\begin{gathered}
\left(\iiint_{\left(\left(1-\frac{1}{n}\right) \frac{1}{\sqrt{n}} B^{n}\right) \times B^{n} \times\left(0, \frac{1}{n^{3 / 2}}\right)} \frac{\left\|f^{x}(u y)-P_{u}^{1} f^{x}(u y)\right\|_{Y}^{\kappa \beta}}{V_{n} u^{1+\kappa \beta}} \mathrm{d} x \mathrm{~d} y \mathrm{~d} u\right)^{\frac{1}{\kappa \beta}} \\
>\left(1-\frac{1}{n}\right)^{\frac{n}{\kappa \beta}}\left(\frac{V_{n}}{n^{\frac{n}{2}}}\right)^{\frac{1}{\kappa \beta}}\left(\int_{\frac{n^{-3 / 2}}{M}}^{n^{-3 / 2}} \frac{\mathrm{d} u}{u}\right)^{\frac{1}{\kappa \beta}} \frac{2 e K \beta^{15} n^{4}}{(\log M)^{\frac{1}{\kappa \beta}}} \geqslant e K \beta^{15} n^{4}\left(\frac{V_{n}}{n^{\frac{n}{2}}}\right)^{\frac{1}{\kappa \beta}},
\end{gathered}
$$

thus contradicting (42). Observe that by Lemma 10 we have

$$
\left\|P_{u}^{1} f^{x}\right\|_{\operatorname{Lip}\left(\mathbb{R}^{n}\right)} \stackrel{(15)}{=}\left\|T_{u} f^{x}\right\|_{X \rightarrow Y} \leqslant\left\|f^{x}\right\|_{\operatorname{Lip}\left(\mathbb{R}^{n}\right)} \leqslant 1 .
$$

Hence, if we set $\Lambda \stackrel{\text { def }}{=} P_{x}^{1} f^{x}$ then $\Lambda: \mathbb{R}^{n} \rightarrow Y$ is affine, $\|\Lambda\|_{\operatorname{Lip}\left(\mathbb{R}^{n}\right)} \leqslant 1$, and by a change of variable one can rewrite (44) as follows.

$$
\left(\frac{1}{\operatorname{vol}\left(x+u B^{n}\right)} \int_{x+u B^{n}}\|f(z)-\Lambda(z)\|_{Y}^{\kappa \beta} \mathrm{d} z\right)^{\frac{1}{\kappa \beta}} \leqslant \frac{2 e K \beta^{15} n^{4} u}{(\log M)^{\frac{1}{\kappa \beta}}} .
$$

This is not quite the type of conclusion that we desire, because the averaging in (45) occurs over a Euclidean ball rather than a ball in $\left(\mathbb{R}^{n},\|\cdot\|_{X}\right)$. We overcome this via another averaging step.

Observe that

$$
x+\left(1-\frac{1}{n}\right) u B^{n}+\frac{u}{n} B_{X} \subseteq x+u B^{n} \subseteq \frac{1}{\sqrt{n}} B^{n} \stackrel{(8)}{\subseteq} B_{X} .
$$

Indeed, if $a, b \in \mathbb{R}^{n}$ satisfy $\|a\|_{2} \leqslant(1-1 / n) u$ and $\|b\|_{X} \leqslant u / n$ then,

$$
\|a+b\|_{2} \leqslant\|a\|_{2}+\|b\|_{2} \stackrel{(8)}{\leqslant}\|a\|_{2}+\|b\|_{X} \leqslant\left(1-\frac{1}{n}\right) u+\frac{u}{n}=u,
$$




\section{Tuomas Hytönen, SEAn LI, AND Assaf NAOR}

Now, define $A \subseteq \mathbb{R}^{n} \times \mathbb{R}^{n}$ as follows.

$$
\begin{aligned}
A & \stackrel{\text { def }}{=}\left\{(y, w): y \in x+\left(1-\frac{1}{n}\right) u B^{n} \wedge w \in y+\frac{u}{n} B_{X}\right\} \\
& =\left\{(y, w): w \in x+\left(1-\frac{1}{n}\right) u B^{n}+\frac{u}{n} B_{X} \wedge y \in\left(x+\left(1-\frac{1}{n}\right) u B^{n}\right) \cap\left(w+\frac{u}{n} B_{X}\right)\right\} .
\end{aligned}
$$

By (46), $f(w)$ is well-defined for every $(y, w) \in A$. It therefore follows from Fubini's theorem that

$$
\begin{aligned}
& \iint_{A}\|f(w)-\Lambda(w)\|_{Y}^{\kappa \beta} \mathrm{d} w \mathrm{~d} y \\
& =\int_{x+\left(1-\frac{1}{n}\right) u B^{n}} \mathrm{~d} y \int_{y+\frac{u}{n} B_{X}}\|f(w)-\Lambda(w)\|_{Y}^{\kappa \beta} \mathrm{d} w \\
& =\int_{x+\left(1-\frac{1}{n}\right) u B^{n}+\frac{u}{n} B_{X}} \operatorname{vol}\left(\left(x+\left(1-\frac{1}{n}\right) u B^{n}\right) \cap\left(w+\frac{u}{n} B_{X}\right)\right)\|f(w)-\Lambda(w)\|_{Y}^{\kappa \beta} \mathrm{d} w \\
& \leqslant \operatorname{vol}\left(\frac{u}{n} B_{X}\right) \int_{x+u B^{n}}\|f(w)-\Lambda(w)\|_{Y}^{\kappa \beta} \mathrm{d} w .
\end{aligned}
$$

Hence,

$$
\begin{aligned}
& \frac{1}{\operatorname{vol}\left(x+\left(1-\frac{1}{n}\right) u B^{n}\right)} \int_{x+\left(1-\frac{1}{n}\right) u B^{n}} \frac{\mathrm{d} y}{\operatorname{vol}\left(y+\frac{u}{n} B_{X}\right)} \int_{y+\frac{u}{n} B_{X}}\|f(w)-\Lambda(w)\|_{Y}^{\kappa \beta} \mathrm{d} w \\
& \leqslant \frac{1}{\operatorname{vol}\left(x+\left(1-\frac{1}{n}\right) u B^{n}\right)} \int_{x+u B^{n}}\|f(w)-\Lambda(w)\|_{Y}^{\kappa \beta} \mathrm{d} w \leqslant \frac{\left(2 e K \beta^{15} n^{4} u\right)^{\kappa \beta}}{\left(1-\frac{1}{n}\right)^{n} \log M} \leqslant \frac{\left(4 e K \beta^{15} n^{4} u\right)^{\kappa \beta}}{\log M} .
\end{aligned}
$$

This implies that there exists $y \in x+\left(1-\frac{1}{n}\right) u B^{n}$ such that

$$
\left(\frac{1}{\operatorname{vol}\left(y+\frac{u}{n} B_{X}\right)} \int_{y+\frac{u}{n} B_{X}}\|f(w)-\Lambda(w)\|_{Y}^{\kappa \beta} \mathrm{d} w\right)^{\frac{1}{\kappa \beta}} \leqslant \frac{4 e K \beta^{15} n^{4} u}{(\log M)^{\frac{1}{\kappa \beta}}}=\frac{4 e K \beta^{15} n^{5}}{(\log M)^{\frac{1}{\kappa \beta}}} \cdot \frac{u}{n}
$$

Recalling Definition (1), since by (43) we are ensured that $u \geqslant 1 /\left(M n^{3 / 2}\right)$, it follows that

$$
\forall M \in(1, \infty), \quad r_{\kappa \beta}^{X \rightarrow Y}\left(\frac{4 e K \beta^{15} n^{5}}{(\log M)^{\frac{1}{\kappa \beta}}}\right) \geqslant \frac{1}{M n^{\frac{3}{2}}} .
$$

For $\varepsilon \in(0,1)$ choose $M=e^{\left(4 e K \beta^{15} n^{5} / \varepsilon\right)^{\kappa \beta}}$ in (47), thus yielding Theorem 5 as follows.

$$
\forall \varepsilon \in(0,1), \quad r_{\kappa \beta}^{X \rightarrow Y}(\varepsilon) \geqslant \frac{1}{n^{\frac{3}{2}}} \exp \left(-\frac{\left(4 e K \beta^{15} n^{5}\right)^{\kappa \beta}}{\varepsilon^{\kappa \beta}}\right) .
$$


QuAntitative AfFine ApProximation FOR UMD TARgEts

\section{Preliminaries on UMD Banach spaces}

This section is devoted to the presentation of several analytic properties of UMD Banach spaces that will be used extensively in the proof of Theorem 5.

Let $\left(Y,\|\cdot\|_{Y}\right)$ be a UMD Banach space and fix $p \in(1, \infty)$. Denote (as usual) by $\beta_{p}(Y)$ the infimum over those $\beta \in(1, \infty]$ such that if $\left\{M_{j}\right\}_{j=0}^{\infty}$ is a $Y$-valued $p$-integrable martingale defined on some probability space $(\Omega, \mathbb{P})$ then for every $n \in \mathbb{N}$ and every $\varepsilon_{1}, \ldots, \varepsilon_{n} \in\{-1,1\}$ we have

$$
\int_{\Omega}\left\|M_{0}+\sum_{j=1}^{n} \varepsilon_{j}\left(M_{j}-M_{j-1}\right)\right\|_{Y}^{p} \mathrm{~d} \mathbb{P} \leqslant \beta^{p} \int_{\Omega}\left\|M_{n}\right\|_{Y}^{p} \mathrm{~d} \mathbb{P} .
$$

Thus, using the notation of the Introduction, we have $\beta_{2}(Y)=\beta(Y)$. The following inequality is well-known; see [17] for its proof.

$$
\beta_{p}(Y) \lesssim \frac{p^{2}}{p-1} \beta(Y)
$$

We also record for future use that (48) implies (see [53, Thm. 4.4]) that for every $a_{0}, a_{1}, \ldots, a_{n} \in \mathbb{R}$,

$$
\int_{\Omega}\left\|a_{0} M_{0}+\sum_{j=1}^{n} a_{j}\left(M_{j}-M_{j-1}\right)\right\|_{Y}^{p} \mathrm{~d} \mathbb{P} \leqslant\left(\max _{j \in\{0, \ldots, n\}}\left|a_{j}\right|^{p}\right) \beta_{p}(X)^{p} \int_{\Omega}\left\|M_{n}\right\|_{Y}^{p} \mathrm{~d} \mathbb{P} .
$$

In [33] Garling introduced two parameters $\beta_{p}^{+}(Y), \beta_{p}^{-}(Y)$, defined to be the best constants in the following inequalities, which are required to hold true for every martingale $\left\{M_{j}\right\}_{j=0}^{\infty}$ as above.

$$
\mathbb{E}_{\varepsilon}\left[\int_{\Omega}\left\|M_{0}+\sum_{j=1}^{n} \varepsilon_{n}\left(M_{j}-M_{j-1}\right)\right\|_{Y}^{p} \mathrm{~d} \mathbb{P}\right] \leqslant \beta_{p}^{+}(Y)^{p} \int_{\Omega}\left\|M_{n}\right\|_{Y}^{p} \mathrm{~d} \mathbb{P},
$$

and

$$
\int_{\Omega}\left\|M_{n}\right\|_{Y}^{p} \mathrm{~d} \mathbb{P} \leqslant \beta_{p}^{-}(Y)^{p} \mathbb{E}_{\varepsilon}\left[\int_{\Omega}\left\|M_{0}+\sum_{j=1}^{n} \varepsilon_{n}\left(M_{j}-M_{j-1}\right)\right\|_{Y}^{p} \mathrm{~d} \mathbb{P}\right],
$$

where $\mathbb{E}_{\varepsilon}[\cdot]$ denotes the expectation with respect to $\varepsilon=\left(\varepsilon_{1}, \ldots, \varepsilon_{n}\right)$ chosen uniformly at random from $\{-1,1\}^{n}$. Garling's inequalities are weaker than (48), which is required to hold for every $\varepsilon \in\{-1,1\}^{n}$ rather than only in expectation with respect to $\varepsilon$. Hence,

$$
\max \left\{\beta_{p}^{+}(Y), \beta_{p}^{-}(Y)\right\} \leqslant \beta_{p}(Y),
$$

but there are examples of Banach spaces $Y$ for which $\beta_{p}^{+}(Y)$ or $\beta_{p}^{-}(Y)$ is markedly smaller than $\beta_{p}(Y)$; see $[33,34]$. Some of the ensuing estimates can be stated in terms of the parameters $\beta_{p}(Y), \beta_{p}^{+}(Y), \beta_{p}^{-}(Y)$, but in order to avoid cumbersome expressions we will sometimes state our bounds in terms of the quantity $\beta(Y):=\beta_{2}(Y)$, by invoking (49) and (51). In fact, in our setting we will always choose $2 \leqslant p \asymp \beta(Y)$, in which case by using (49) and (51) we will sometimes bound from above the quantities $\beta_{p}(Y), \beta_{p}^{+}(Y), \beta_{p}^{-}(Y)$ by a universal constant multiple of $p \beta(Y) \asymp \beta(Y)^{2}$. This choice has the advantage of simplifying some of the ensuing discussion, but it yields bounds that could be improved for some (quite exotic) UMD Banach spaces $Y$ by a straightforward inspection of our proofs. 


\section{1 $\mathscr{R}$-boundedness}

Let $\left(X,\|\cdot\|_{X}\right)$ and $\left(Y,\|\cdot\|_{Y}\right)$ be Banach spaces. The space of bounded linear operators from $X$ to $Y$ is denoted $\mathscr{L}(X, Y)$. Following [10,21], a set of operators $\mathcal{T} \subseteq \mathscr{L}(X, Y)$ is said to be $\mathscr{R}$-bounded if

$$
\mathbb{E}_{\varepsilon}\left[\left\|\sum_{j=1}^{N} \varepsilon_{j} T_{j} x_{j}\right\|_{Y}^{p}\right] \leqslant C^{p} \mathbb{E}_{\varepsilon}\left[\left\|\sum_{j=1}^{N} \varepsilon_{j} x_{j}\right\|_{X}^{p}\right],
$$

for every $N \in \mathbb{N}$, every $x_{1}, \ldots, x_{N} \in Y$ and every $T_{1}, \ldots, T_{N} \in \mathcal{T}$, and some (equivalently, by Kahane's inequality [50], for all) $p \in[1, \infty)$. The infimum over those $C$ in (52) is denoted $\mathscr{R}_{p}(\mathcal{T})$.

The following result is due to Bourgain [12]; see also [30] for a proof.

Proposition 20 (Bourgain's vector-valued Stein inequality). Suppose that $\left(Y,\|\cdot\|_{Y}\right)$ is a UMD Banach space and fix $p \in(1, \infty)$. Let $\left\{\mathscr{F}_{j}\right\}_{j \in \mathbb{Z}}$ be an increasing sequence of sub- $\sigma$-algebras on some probability space $(\Omega, \mathscr{F}, \mathbb{P})$. For every $j \in \mathbb{Z}$ let $\mathscr{E}_{j} \in \mathscr{L}\left(L_{p}(\mathbb{P}, Y), L_{p}(\mathbb{P}, Y)\right)$ be the conditional expectation operator corresponding to $\mathscr{F}_{j}$, i.e., $\mathscr{E}_{j} f=\mathbb{E}\left[f \mid \mathscr{F}_{j}\right]$ for every $f \in L_{p}(\mathbb{P}, Y)$. Then

$$
\mathscr{R}_{p}\left(\left\{\mathscr{E}_{j}\right\}_{j \in \mathbb{Z}}\right) \leqslant \beta_{p}^{+}(Y)
$$

By a classical representation theorem for positive self-adjoint semigroups of contractions due to Rota [70] (see also [73, Sec. VI] or [59, Thm. 2.5]), Proposition 20 implies the following dimension independent $\mathscr{R}$-boundedness estimate for the heat semigroup. Here and in what follows, $\Delta$ denotes the Laplacian on $\mathbb{R}^{n}$.

Corollary 21 ( $\mathscr{R}$-boundedness of the heat semigroup). Let $\left(Y,\|\cdot\|_{Y}\right)$ be a UMD Banach space, $n \in \mathbb{N}$, and $p \in(1, \infty)$. Then

$$
\mathscr{R}_{p}\left(\left\{e^{t \Delta}\right\}_{t \in(0, \infty)}\right) \leqslant \beta_{p}^{+}(Y) \text {. }
$$

Proof. For any $\delta>0$, the operator $Q=e^{\frac{1}{2} \delta \Delta}$ satisfies the assumptions of Rota's theorem as formulated in [73, Sec. VI] or [59, Thm. 2.5]. Thus there exists a measure space $(\Omega, \mathscr{F}, \mu)$ with an increasing sequence of sub- $\sigma$-algebras $\left\{\mathscr{F}_{j}\right\}_{j \in \mathbb{Z}}$ and yet another sub- $\sigma$-algebra $\mathscr{F}^{\prime}$ with the corresponding conditional expectation operators $\mathscr{E}_{j}$ and $\mathscr{E}^{\prime}$ such that

$$
\forall j \in \mathbb{N} \cup\{0\}, \quad e^{j \delta \Delta}=Q^{2 j}=J^{-1} \mathscr{E}^{\prime} \mathscr{E}_{-j} J,
$$

where $J: L_{p}\left(\mathbb{R}^{n}, Y\right) \rightarrow L_{p}\left(\Omega, \mathscr{F}^{\prime}, Y\right)$ is an isometric isomorphism. Thus

$$
\begin{aligned}
& \mathscr{R}_{p}\left(\left\{e^{j \delta \Delta}\right\}_{j \in \mathbb{N} \cup\{0\}}\right) \\
& =\mathscr{R}_{p}\left(\left\{J \mathscr{E}^{\prime} \mathscr{E}_{-j} J\right\}_{j \in \mathbb{N} \cup\{0\}}\right) \\
& \leqslant\left\|J^{-1}\right\|_{L_{p}\left(\Omega, \mathscr{F}^{\prime}, Y\right) \rightarrow L_{p}\left(\mathbb{R}^{n}, Y\right)} \cdot\left\|\mathscr{E}^{\prime}\right\|_{L_{p}(\Omega, \mathscr{F}, \mu) \rightarrow L_{p}\left(\Omega, \mathscr{F}^{\prime}, \mu\right)} \cdot \mathscr{R}_{p}\left(\left\{\mathscr{E}_{-j}\right\}_{j \in \mathbb{N} \cup\{0\}}\right) \cdot\|J\|_{L_{p}\left(\mathbb{R}^{n}, Y\right) \rightarrow L_{p}\left(\Omega, \mathscr{F}^{\prime}, Y\right)} \\
& \leqslant \beta_{+}(Y),
\end{aligned}
$$

where we used Proposition 20 together with easy properties of $\mathscr{R}$-bounds and the contractivity of conditional expectations. 


\section{QuAntitative AfFine ApProximation For UMD TARGETS}

Using basic properties of $\mathscr{R}$-bounds (cf. [76, Proposition 9.5]), if $\mathscr{T}=\bigcup_{k=1}^{\infty} \mathscr{T}_{k}$ is a union of an increasing sequence $\mathscr{T}_{k} \subseteq \mathscr{T}_{k+1}$, and $\mathscr{T}$ is the closure of $\mathscr{T}$ in the strong operator topology, then $\mathscr{R}_{p}(\overline{\mathscr{T}})=\mathscr{R}_{p}(\mathscr{T})=\lim _{k \rightarrow \infty} \mathscr{R}_{p}\left(\mathscr{T}_{k}\right)$. With $\mathscr{T}_{k}=\left\{e^{j 2^{-k} \Delta}\right\}_{j \in \mathbb{N} \cup\{0\}}$, we have $\overline{\mathscr{T}}=\left\{e^{t \Delta}\right\}_{t \in(0, \infty)}$ by the strong continuity of $t \mapsto e^{t \Delta}$, and this proves the claim.

Our next goal is to prove Theorem 24 below, which is a useful bound on the norm of operators on UMD Banach spaces that admit a certain integral representation in terms of the heat semigroup. Results in this spirit have been implicitly used for a long time; see for examples the probabilistic treatment of the Riesz transforms by Gundy and Varopoulos [37], and of the Beurling-Ahlfors transform by Bañuelos and Méndez-Hernández [4]. Formulations in the UMD-valued setting appear in [41, 42, 43]. The version below is essentially a combination of some of these earlier results but it does not appear as stated in the literature, so its proof is included here.

In what follows, we will use some aspects of the theory of vector-valued stochastic integration with respect to a Brownian motion $\left\{B(t)=\left(B_{1}(t), \ldots, B_{n}(t)\right)\right\}_{t \in(0, \infty)}$ in $\mathbb{R}^{n}$, starting at 0 . Here and below, a Brownian motion in $\mathbb{R}^{n}$ is always understood to be a standard Brownian motion.

It should be noted that for our purposes it is enough to consider finite-dimensional-valued functions, in which case the stochastic integrals can be defined coordinate-wise in the classical sense. We refer to [51, Chapter 3] for the relevant background (and much more). It might be helpful to note that the formulae in [51], which often involve the quadratic variation $\langle M, N\rangle_{t}$ of two stochastic processes $M$ and $N$, take a simpler form in the Brownian case of our interest, by using the identities $\left\langle B_{i}, B_{j}\right\rangle_{t}=\delta_{i j} t$ (see [51, Theorem 3.3.16]).

In particular, Itô's formula (see [51, Theorem 3.3.6]) is valid in our setting, since it holds true for each scalar-valued coordinate function. For a comprehensive theory of vector-valued stochastic integration, whose full strength is not needed here, see [78, 77].

Before stating Theorem 24 we describe some preliminary background and simple estimates that will be used in its proof. First, we recall the following decoupling inequalities due to Garling [32].

Theorem 22 (Garling's decoupling inequalities). For $n \in \mathbb{N}$, let $\left\{B(t)=\left(B_{1}(t), \ldots, B_{n}(t)\right)\right\}_{t \in(0, \infty)}$ be a Brownian motion in $\mathbb{R}^{n}$, starting at 0 . Also, let $\left\{C(t)=\left(C_{1}(t), \ldots, C_{n}(t)\right)\right\}_{t \in(0, \infty)}$ be an independent copy of $\{B(t)\}_{t \in(0, \infty)}$. Suppose that $\left(Y,\|\cdot\|_{Y}\right)$ is a UMD Banach space and $p \in(1, \infty)$. Let $V=\left(V_{1}, \ldots, V_{n}\right)$ : $(0, \infty) \rightarrow Y^{n}$ be a stochastic process that is adapted to the same filtration as $\left.\{B(t))\right\}_{t \in(0, \infty)}$, takes values in a finite-dimensional subspace of $Y^{n}$, and satisfies

$$
\mathbb{E}\left[\left(\int_{0}^{\infty} \sum_{j=1}^{n}\left\|V_{j}(t)\right\|_{Y}^{2} \mathrm{~d} t\right)^{\frac{p}{2}}\right]<\infty
$$

The finite dimensionality assumption and the integrability assumption (53) guarantee the existence of the stochastic integrals below by the scalar-valued theory. Then

$$
\mathbb{E}\left[\left\|\int_{0}^{\infty} \sum_{j=1}^{n} V_{j}(t) \mathrm{d} B_{j}(t)\right\|_{Y}^{p}\right] \leqslant \beta_{p}^{-}(Y)^{p} \mathbb{E}\left[\left\|\int_{0}^{\infty} \sum_{j=1}^{n} V_{j}(t) \mathrm{d} C_{j}(t)\right\|_{Y}^{p}\right],
$$

and

$$
\mathbb{E}\left[\left\|\int_{0}^{\infty} \sum_{j=1}^{n} V_{j}(t) \mathrm{d} C_{j}(t)\right\|_{Y}^{p}\right] \leqslant \beta_{p}^{+}(Y)^{p} \mathbb{E}\left[\left\|\int_{0}^{\infty} \sum_{j=1}^{n} V_{j}(t) \mathrm{d} B_{j}(t)\right\|_{Y}^{p}\right] .
$$




\section{TuOmas Hytönen, SEAn Li, AND AsSaf NaOR}

Continuing with the notation of Theorem 22 , for every (operator-valued) measurable ${ }^{1}$ mapping $\Phi:(0, \infty) \rightarrow \mathscr{L}(Y, Y)$ we have

$$
\mathbb{E}\left[\left\|\int_{0}^{\infty} \sum_{j=1}^{n} \Phi(t) V_{j}(t) \mathrm{d} C_{j}(t)\right\|_{Y}^{p}\right] \leqslant \mathscr{R}_{p}(\Phi)^{p} \mathbb{E}\left[\left\|\int_{0}^{\infty} \sum_{j=1}^{n} V_{j}(t) \mathrm{d} C_{j}(t)\right\|_{Y}^{p}\right],
$$

where we use the notation

$$
\mathscr{R}_{p}(\Phi) \stackrel{\text { def }}{=} \mathscr{R}_{p}\left(\{\Phi(t)\}_{t \in(0, \infty)}\right) .
$$

The estimate (54) follows directly from the definition of $\mathscr{R}$-boundedness by approximating the integrals by Riemann sums; see Exercise 4 in Section 9.4 of [76]. Alternatively, inequality (54) follows by combining Theorem 6.14 and Theorem 9.13 of [76]. By (54) and Theorem 22 we see that

$$
\mathbb{E}\left[\left\|\int_{0}^{\infty} \sum_{j=1}^{n} \Phi(t) V_{j}(t) \mathrm{d} B_{j}(t)\right\|_{Y}^{p}\right] \leqslant \beta_{p}^{+}(Y)^{p} \beta_{p}^{-}(Y)^{p} \mathscr{R}_{p}(\Phi)^{p} \mathbb{E}\left[\left\|\int_{0}^{\infty} \sum_{j=1}^{n} V_{j}(t) \mathrm{d} B_{j}(t)\right\|_{Y}^{p}\right] .
$$

In the same vein as the above discussion, by approximating the integrals by Riemann sums it follows from (50) that if $\phi:(0, \infty) \rightarrow \mathbb{R}$ is measurable then

$$
\mathbb{E}\left[\left\|\int_{0}^{\infty} \sum_{j=1}^{n} \phi(t) V_{j}(t) \mathrm{d} B_{j}(t)\right\|_{Y}^{p}\right] \leqslant \beta_{p}(Y)^{p}\|\phi\|_{L_{\infty}(0, \infty)}^{p} \mathbb{E}\left[\left\|\int_{0}^{\infty} \sum_{j=1}^{n} V_{j}(t) \mathrm{d} B_{j}(t)\right\|_{Y}^{p}\right] .
$$

We record for future use the following simple estimate.

Lemma 23. Fix $n \in \mathbb{N}$ and let $\left\{B(t)=\left(B_{1}(t), \ldots, B_{n}(t)\right)\right\}_{t \in[0, \infty)}$ be a Brownian motion in $\mathbb{R}^{n}$, starting at 0 . For every Banach space $\left(Y,\|\cdot\|_{Y}\right)$, every $p \in(1, \infty)$ and every smooth and compactly supported $h: \mathbb{R}^{n} \rightarrow Y$ with a finite-dimensional range, we have

$$
\limsup _{\tau \rightarrow \infty}\left(\int_{\mathbb{R}^{n}} \mathbb{E}\left[\left\|\sum_{j=1}^{n} \int_{0}^{\tau} \frac{\partial}{\partial x_{j}} e^{\frac{\tau-t}{2} \Delta} h(B(t)+x) \mathrm{d} B_{j}(t)\right\|_{Y}^{p}\right] \mathrm{d} x\right)^{\frac{1}{p}} \leqslant\|h\|_{L_{p}\left(\mathbb{R}^{n}, Y\right)} .
$$

Proof. If $\varphi \in L_{1}\left(\mathbb{R}^{n}\right)$ then $\mathbb{E}[\varphi(B(t)+x)]=e^{\frac{t}{2} \Delta} \varphi(x)$ for every $t \in[0, \infty)$ and $x \in \mathbb{R}^{n}$. Hence,

$$
\int_{\mathbb{R}^{n}} \mathbb{E}[\varphi(B(t)+x)] \mathrm{d} x=\int_{\mathbb{R}^{n}} e^{\frac{t}{2} \Delta} \varphi(x) \mathrm{d} x=\int_{\mathbb{R}^{n}} \varphi(x) \mathrm{d} x .
$$

For $(x, t) \in \mathbb{R}^{n} \times(0, \infty)$, let $u(x, t) \stackrel{\text { def }}{=} e^{\frac{t}{2} \Delta} h(x)$ denote the heat extension of $h$. By Itô's formula (see [51, Theorem 3.3.6]) applied to the function $t \mapsto u(B(t)+x, \tau-t)$, for every $x \in \mathbb{R}^{n}$ we have

$$
\begin{aligned}
& \sum_{j=1}^{n} \int_{0}^{\tau} \frac{\partial u}{\partial x_{j}}(B(t)+x, \tau-t) \mathrm{d} B_{j}(t) \\
& =u(B(\tau)+x, 0)-u(x, \tau)-\int_{0}^{\tau}\left(-\frac{\partial u}{\partial t}+\frac{1}{2} \Delta_{x} u\right)(B(t)+x, \tau-t) \mathrm{d} t \\
& =h(B(\tau)+x)-e^{\frac{\tau}{2} \Delta} h(x) .
\end{aligned}
$$

\footnotetext{
${ }^{1}$ Here, and in what follows, given two Banach spaces $\left(X,\|\cdot\|_{X}\right)$ and $\left(Y,\|\cdot\|_{Y}\right)$ and an open subset $\Omega \subseteq \mathbb{R}^{n}$, when we say that an operator-valued mapping $\Phi: \Omega \rightarrow \mathscr{L}(X, Y)$ is measurable we mean measurability in the strong operator topology, i.e., we require that for every $x \in X$ the mapping $w \mapsto \Phi(w) x$ from $\Omega$ to $Y$ has the property that the inverse image of every Borel subset of $Y$ is Lebesgue-measurable.
} 
Consequently,

$$
\begin{aligned}
& \left(\int_{\mathbb{R}^{n}} \mathbb{E}\left[\left\|\sum_{j=1}^{n} \int_{0}^{\tau} \frac{\partial}{\partial x_{j}} e^{\frac{\tau-t}{2} \Delta} h(B(t)+x) \mathrm{d} B_{j}(t)\right\|_{Y}^{p}\right] \mathrm{d} x\right)^{\frac{1}{p}} \\
& \stackrel{(60)}{\leqslant}\left(\int_{\mathbb{R}^{n}} \mathbb{E}\left[\|h(B(\tau)+x)\|_{Y}^{p}\right] \mathrm{d} x\right)^{\frac{1}{p}}+\left(\int_{\mathbb{R}^{n}} \mathbb{E}\left[\left\|e^{\frac{\tau}{2} \Delta} h(x)\right\|_{Y}^{p}\right] \mathrm{d} x\right)^{\frac{1}{p}} \\
& \stackrel{(59)}{=}\|h\|_{L_{p}\left(\mathbb{R}^{n}, Y\right)}+\left\|e^{\frac{\tau}{2} \Delta} h\right\|_{L_{p}\left(\mathbb{R}^{n}, Y\right)} .
\end{aligned}
$$

To deduce the desired bound (58) from (62), it remains to note that

$$
\forall p \in(1, \infty), \quad \lim _{\tau \rightarrow \infty}\left\|e^{\frac{\tau}{2} \Delta} h\right\|_{L_{p}\left(\mathbb{R}^{n}, Y\right)}=0 .
$$

Indeed, by Young's inequality we have the following point-wise estimate

$$
\forall x \in \mathbb{R}^{n}, \quad\left\|e^{\frac{\tau}{2} \Delta} h(x)\right\|_{Y}=\left\|k_{\frac{\tau}{2}} * h(x)\right\|_{Y} \leqslant\left\|k_{\frac{\tau}{2}}\right\|_{L_{q}\left(\mathbb{R}^{n}\right)}\|h\|_{L_{p}\left(\mathbb{R}^{n}, Y\right)},
$$

where $k: \mathbb{R}^{n} \rightarrow \mathbb{R}$ is the heat kernel and $q=p /(p-1)$. Since $q \in(0, \infty)$, the $L_{q}$-norm of the heat kernel $k_{\tau / 2}$ converges to 0 as $\tau \rightarrow \infty$. Moreover, $\left\|e^{\frac{\tau}{2} \Delta} h\right\|_{Y}$ is dominated point-wise by the Hardy-Littlewood maximal function $M\|h\|_{Y} \in L_{p}\left(\mathbb{R}^{n}\right)$. Hence (63) follows from (64) by an application of the dominated convergence theorem.

The following theorem is the main result of the present section: it establishes an estimate that will be used several times in the ensuing discussion.

Theorem 24. Fix $n \in \mathbb{N}$ and $p \in(1, \infty)$. Suppose that for $t \in(0, \infty)$ we are given a bounded operator $A(t): Y \rightarrow Y$ such that the mapping $A:(0, \infty) \rightarrow \mathscr{L}(Y, Y)$ is bounded and measurable. Also, suppose that $T: L_{p}\left(\mathbb{R}^{n}, Y\right) \rightarrow L_{p}\left(\mathbb{R}^{n}, Y\right)$ is a linear operator that has the following dual representation. For every sufficiently nice $f$ in a dense subspace of $L_{p}\left(\mathbb{R}^{n}, Y\right)$, and $g^{*}$ in a dense subspace of $L_{q}\left(\mathbb{R}^{n}, Y^{*}\right)$, where $q=p /(p-1)$, we have

$$
\int_{\mathbb{R}^{n}} g^{*}(x)(T f(x)) \mathrm{d} x=\sum_{j=1}^{n} \int_{0}^{\infty} \int_{\mathbb{R}^{n}}\left(\frac{\partial}{\partial x_{j}} e^{\frac{t}{2} \Delta} g^{*}(x)\right)\left(A(t) \frac{\partial}{\partial x_{j}} e^{\frac{t}{2} \Delta} f(x)\right) \mathrm{d} x \mathrm{~d} t .
$$

Then, recalling the notation (55),

$$
\|T\|_{L_{p}\left(\mathbb{R}^{n}, Y\right) \rightarrow L_{p}\left(\mathbb{R}^{n}, Y\right)} \leqslant \beta_{p}^{+}(Y) \beta_{p}^{-}(Y) \mathscr{R}_{p}(A) .
$$

Moreover, in the special case $A:(0, \infty) \rightarrow \mathbb{C}$ we have

$$
\|T\|_{L_{p}\left(\mathbb{R}^{n}, Y\right) \rightarrow L_{p}\left(\mathbb{R}^{n}, Y\right)} \leqslant \beta_{p}(Y)\|A\|_{L_{\infty}(0, \infty)} .
$$




\section{TuOmas Hytönen, SEAn Li, AND AsSaf NaOR}

Proof. By duality and the identity (65), the desired estimate (66) would follow if we show that for every sufficiently nice $f \in L_{p}\left(\mathbb{R}^{n}, Y\right)$ and $g^{*} \in L_{q}\left(\mathbb{R}^{n}, Y^{*}\right)$,

$$
\begin{aligned}
\limsup _{\tau \rightarrow \infty} \sum_{j=1}^{n} \int_{0}^{\tau} \int_{\mathbb{R}^{n}}\left(\frac{\partial}{\partial x_{j}} e^{\frac{s}{2} \Delta} g^{*}(x)\right)\left(A(s) \frac{\partial}{\partial x_{j}} e^{\frac{s}{2} \Delta} f(x)\right) \mathrm{d} x \mathrm{~d} s & \\
& \leqslant \beta_{p}^{+}(Y) \beta_{p}^{-}(Y) \mathscr{R}_{p}(A)\|f\|_{L_{p}\left(\mathbb{R}^{n}, Y\right)}\left\|g^{*}\right\|_{L_{q}\left(\mathbb{R}^{n}, Y^{*}\right)} .
\end{aligned}
$$

Let us consider $f, g$ smooth and compactly supported, and taking values in finite-dimensional subspaces of $Y$ and $Y^{*}$, respectively.

For every $(x, s) \in \mathbb{R}^{n} \times(0, \infty)$ denote for the sake of simplicity

$$
\gamma_{j}^{*}(x, s) \stackrel{\text { def }}{=} \frac{\partial}{\partial x_{j}} e^{\frac{s}{2} \Delta} g^{*}(x) \quad \text { and } \quad \phi_{j}(x, s) \stackrel{\text { def }}{=} A(s) \frac{\partial}{\partial x_{j}} e^{\frac{s}{2} \Delta} f(x) .
$$

Let $\left\{B(t)=\left(B_{1}(t), \ldots, B_{n}(t)\right)\right\}_{t \in[0, \infty)}$ be a Brownian motion in $\mathbb{R}^{n}$, starting at 0 . It follows from the identity (59) that for every $\tau \in(0, \infty)$,

$$
\begin{aligned}
& \sum_{j=1}^{n} \int_{0}^{\tau} \int_{\mathbb{R}^{n}}\left(\frac{\partial}{\partial x_{j}} e^{\frac{s}{2} \Delta} g^{*}(x)\right)\left(A(s) \frac{\partial}{\partial x_{j}} e^{\frac{s}{2} \Delta} f(x)\right) \mathrm{d} x \mathrm{~d} s \\
& \quad=\int_{\mathbb{R}^{n}} \mathbb{E}\left[\sum_{j=1}^{n} \int_{0}^{\tau} \gamma_{j}^{*}(B(t)+x, \tau-t)\left(\phi_{j}(B(t)+x, \tau-t)\right) d t\right] \mathrm{d} x=\int_{\mathbb{R}^{n}} \mathbb{E}\left[G_{\tau}^{*}(x)\left(F_{\tau}(x)\right)\right] \mathrm{d} x,
\end{aligned}
$$

where we introduce the notations

$$
F_{\tau}(x) \stackrel{\text { def }}{=} \sum_{j=1}^{n} \int_{0}^{\tau} A(\tau-t) \frac{\partial}{\partial x_{j}} e^{\frac{\tau-t}{2} \Delta} f(B(t)+x) \mathrm{d} B_{j}(t),
$$

and

$$
G_{\tau}^{*}(x) \stackrel{\text { def }}{=} \sum_{j=1}^{n} \int_{0}^{\tau} \frac{\partial}{\partial x_{j}} e^{\frac{\tau-t}{2} \Delta} g^{*}(B(t)+x) \mathrm{d} B_{j}(t) .
$$

(69) is a well-known identity (see [51, Proposition 2.17]) for scalar-valued functions, and it follows from this for the vector-valued functions with a finite-dimensional range that we consider here.

By Hölder's inequality it follows from (69) that

$$
\begin{aligned}
\sum_{j=1}^{n} \int_{0}^{\tau} \int_{\mathbb{R}^{n}}\left(\frac{\partial}{\partial x_{j}} e^{\frac{s}{2} \Delta} g^{*}(x)\right)\left(A(s) \frac{\partial}{\partial x_{j}} e^{\frac{s}{2} \Delta} f(x)\right) \mathrm{d} x \mathrm{~d} s & \\
& \leqslant\left(\int_{\mathbb{R}^{n}} \mathbb{E}\left[\left\|F_{\tau}(x)\right\|_{Y}^{p}\right] \mathrm{d} x\right)^{\frac{1}{p}}\left(\int_{\mathbb{R}^{n}} \mathbb{E}\left[\left\|G_{\tau}^{*}(x)\right\|_{Y^{*}}^{q}\right] \mathrm{d} x\right)^{\frac{1}{q}} .
\end{aligned}
$$

Recalling (71), by Lemma 23 we have

$$
\limsup _{\tau \rightarrow \infty}\left(\int_{\mathbb{R}^{n}} \mathbb{E}\left[\left\|G_{\tau}^{*}(x)\right\|_{Y^{*}}^{q}\right] \mathrm{d} x\right)^{\frac{1}{q}} \leqslant\left\|g^{*}\right\|_{L_{q}\left(\mathbb{R}^{n}, Y^{*}\right)} .
$$


QuAntitative AfFine ApProximation FOR UMD TARgEts

Recalling (70), it follows from (56) and (57) that if we set $K=\beta_{p}^{+}(Y) \beta_{p}^{-}(Y) \mathscr{R}_{p}(A)$ if $Y \neq \mathbb{R}$, and $K=\beta_{p}(Y)\|A\|_{L_{\infty}(0, \infty)}$ if $A$ is scalar-valued, then

$$
\int_{\mathbb{R}^{n}} \mathbb{E}\left[\left\|F_{\tau}(x)\right\|_{Y}^{p}\right] \mathrm{d} x \leqslant K^{p} \mathbb{E}\left[\left\|\sum_{j=1}^{n} \int_{0}^{\tau} \frac{\partial}{\partial x_{j}} e^{\frac{\tau-t}{2} \Delta} f(B(t)+x) \mathrm{d} B_{j}(t)\right\|_{Y}^{p}\right] .
$$

Another application of Lemma 23 now implies that

$$
\limsup _{\tau \rightarrow \infty}\left(\int_{\mathbb{R}^{n}} \mathbb{E}\left[\left\|F_{\tau}(x)\right\|_{Y}^{p}\right] \mathrm{d} x\right)^{\frac{1}{p}} \leqslant\|f\|_{L_{q}\left(\mathbb{R}^{n}, Y\right)} .
$$

The desired estimate (68) is a consequence of (72), (73) and (74).

\subsection{Vector-valued multipliers}

Let $\left(Y,\|\cdot\|_{Y}\right)$ be a Banach space. The Fourier transform of $f \in L_{1}\left(\mathbb{R}^{n}, Y\right)$ will be denoted below by $\mathcal{F} f: \mathbb{R}^{n} \rightarrow Y$, where we use the normalization

$$
\mathcal{F} f(x) \stackrel{\text { def }}{=} \frac{1}{(2 \pi)^{n / 2}} \int_{\mathbb{R}^{n}} e^{-i\langle x, y\rangle} f(x) \mathrm{d} y
$$

A possible formulation of Parseval's identity in this vector-valued setting is to say that for functions $f: \mathbb{R}^{n} \rightarrow Y$ and $g^{*}: \mathbb{R}^{n} \rightarrow Y^{*}$ that are either smooth and compactly supported, or Fourier transforms of such functions, we have

$$
\int_{\mathbb{R}^{n}} \mathcal{F} g^{*}(x)(\mathcal{F} f(x)) \mathrm{d} x=\int_{\mathbb{R}^{n}} g^{*}(x)(f(-x)) \mathrm{d} x .
$$

If $\left(X,\|\cdot\|_{X}\right)$ is an additional Banach space and $\mathfrak{m}: \mathbb{R}^{n} \rightarrow \mathscr{L}(X, Y)$ is measurable then the multiplier associated to $\mathfrak{m}$ is defined as usual by considering for every smooth and compactly supported $f: \mathbb{R}^{n} \rightarrow X$ (or the Fourier transform of such a function) the function $T_{\mathfrak{m}} f: \mathbb{R}^{n} \rightarrow Y$ given by

$$
T_{\mathfrak{m}} f \stackrel{\text { def }}{=}\left(\mathcal{F}^{-1} \mathfrak{m}\right) * f=\mathcal{F}^{-1}(x \mapsto \mathfrak{m}(x) \mathcal{F} f(x)) .
$$

If $\mathfrak{m}$ is smooth and locally bounded at least away from the coordinate hyperplanes, and the Fourier transform of $f: \mathbb{R}^{n} \rightarrow X$ is smooth and compactly supported away from these hyperplanes, then also $T_{\mathfrak{m}} f$ has a smooth and compactly supported Fourier transform. Hence, for such $f$ and smooth and compactly supported $g^{*}: \mathbb{R}^{n} \rightarrow Y^{*}$ (or the Fourier transform of such a function), Parseval's identity applies and gives

$$
\int_{\mathbb{R}^{n}} g^{*}(x)\left(T_{\mathfrak{m}} f(x)\right) \mathrm{d} x=\int_{\mathbb{R}^{n}} \mathcal{F} g^{*}(x)(\mathfrak{m}(-x) \mathcal{F} f(-x)) \mathrm{d} x .
$$

Also, under our choice of normalization of the Fourier transform, $-\Delta=T_{\mathfrak{m}}$ for $\mathfrak{m}(x)=\|x\|_{2}^{2}$.

Theorem 24 can be used to bound the following multipliers, which arise as Laplace transforms of $-\Delta$. (Another approach to such multipliers appears in the recent survey [25, Section 2.2.1]; while it is 
presented there for scalar-valued functions, it is based on principles that are valid in any UMD space.) Suppose that $A:(0, \infty) \rightarrow \mathscr{L}(Y, Y)$ is measurable and define $\alpha: \mathbb{R} \rightarrow \mathscr{L}(Y, Y)$ by

$$
\forall y \in Y, \quad \alpha(s) \stackrel{\text { def }}{=} s \int_{0}^{\infty} e^{-s t} A(t) y \mathrm{~d} t .
$$

Then Theorem 24 applies to $\alpha(-\Delta)$, i.e., to the operator $T_{\mathfrak{m}}$ where $\mathfrak{m}: \mathbb{R}^{n} \rightarrow \mathscr{L}(Y, Y)$ is given by

$$
\forall(x, y) \in \mathbb{R}^{n} \times Y, \quad \mathfrak{m}(x) y=\alpha\left(\|x\|_{2}^{2}\right) y=\|x\|_{2}^{2} \int_{0}^{\infty} e^{-t\|x\|_{2}^{2}} A(t) y \mathrm{~d} t .
$$

Indeed, by Parseval's identity (76), the representation (65) is a direct consequence of (77). We therefore have the following dimension independent bound, which holds true for every $p \in(1, \infty)$.

$$
\left\|\Delta \int_{0}^{\infty} e^{t \Delta} A(t) \mathrm{d} t\right\|_{L_{p}\left(\mathbb{R}^{n}, Y\right) \rightarrow L_{p}\left(\mathbb{R}^{n}, Y\right)} \leqslant \beta_{p}(Y)^{2} \mathscr{R}_{p}(A),
$$

where we recall the notation (55). Also, if $A$ takes values in $\mathbb{C}$ then

$$
\left\|\Delta \int_{0}^{\infty} e^{t \Delta} A(t) \mathrm{d} t\right\|_{L_{p}\left(\mathbb{R}^{n}, Y\right) \rightarrow L_{p}\left(\mathbb{R}^{n}, Y\right)} \leqslant \beta_{p}(Y)\|A\|_{L_{\infty}(0, \infty)} .
$$

Later we shall use (78) as a source of dimension-independent bounds for multipliers that correspond to imaginary powers of the Laplacian. Specifically, for every $s \in(0, \infty)$ and $u \in \mathbb{R}$,

$$
s^{i u}=s^{-(1-i u)} s=\frac{s}{\Gamma(1-i u)} \int_{0}^{\infty} t^{-i u} e^{-s t} \mathrm{~d} t .
$$

It therefore follows from (78) that

$$
\left\|(-\Delta)^{i u}\right\|_{L_{p}\left(\mathbb{R}^{n}, Y\right) \rightarrow L_{p}\left(\mathbb{R}^{n}, Y\right)} \leqslant \frac{\beta_{p}(Y)}{|\Gamma(1-i u)|} \asymp \beta_{p}(Y) \frac{e^{|u| \arctan |u|}}{\sqrt{1+|u|}} \asymp \beta_{p}(Y) \frac{e^{\frac{\pi|u|}{2}}}{\sqrt{1+|u|}},
$$

where the penultimate step in (79) is a consequence of Stirling's formula. For ease of later reference, we record the bound that we have just proved as Corollary 25 below. The reverse implication, i.e., that the boundedness $(-\Delta)^{i u}$ on $L_{p}\left(\mathbb{R}^{n}, Y\right)$ implies that $Y$ is UMD, is also true; in fact it was pointed out in [41] that [36] implicitly contains the estimate $\beta_{p}(Y) \leqslant \liminf _{u \rightarrow 0}\left\|(-\Delta)^{i u}\right\|_{L_{p}\left(\mathbb{R}^{n}, Y\right) \rightarrow L_{p}\left(\mathbb{R}^{n}, Y\right)}$.

Corollary 25. Suppose that $p \in(1, \infty)$ and $\left(Y,\|\cdot\|_{Y}\right)$ is a UMD Banach space. Then for every $u \in \mathbb{R}$ and $n \in \mathbb{N}$ we have

$$
\left\|(-\Delta)^{i u}\right\|_{L_{p}\left(\mathbb{R}^{n}, Y\right) \rightarrow L_{p}\left(\mathbb{R}^{n}, Y\right)} \lesssim \beta_{p}(Y) \frac{e^{\frac{\pi|u|}{2}}}{\sqrt{1+|u|}} .
$$

Our next corollary of Theorem 24 is a dimension-independent bound for a multiplier that will be used in the ensuing proof of Theorem 5. We shall use below the following integral representation.

$$
\forall(\theta, \alpha) \in(0,1) \times[0, \infty), \quad \frac{1}{(1+\alpha)^{\theta}}=\frac{\sin (\pi \theta)}{\pi} \int_{0}^{1} \frac{\mathrm{d} s}{s^{1-\theta}(1-s)^{\theta}(1+\alpha s)} .
$$

To verify the validity of (80), simply apply the change of variable $s=t /(1+\alpha-\alpha t)$. 
Corollary 26. Fix $a \in(0,2]$ and $n \in \mathbb{N}$. Define $\mathfrak{m}_{a}: \mathbb{R}^{n} \rightarrow \mathbb{R}$ by setting

$$
\forall, x=\left(x_{1}, \ldots, x_{n}\right) \in \mathbb{R}^{n} \backslash\{0\}, \quad \mathfrak{m}_{a}(x) \stackrel{\text { def }}{=} \frac{\left|x_{1}\right|^{a}}{\|x\|_{2}^{a}} .
$$

Suppose that $\left(Y,\|\cdot\|_{Y}\right)$ is a UMD Banach space and that $p \in(1, \infty)$. Then

$$
\left\|T_{\mathfrak{m}_{a}}\right\|_{L_{p}\left(\mathbb{R}^{n}, Y\right) \rightarrow L_{p}\left(\mathbb{R}^{n}, Y\right)} \leqslant \beta_{p}^{+}(Y)^{2} \beta_{p}^{-}(Y) \leqslant \beta_{p}(Y)^{3} .
$$

Remark 27. When $Y=\mathbb{C}$ in Corollary 26, Bañuelos and Bogdan [3] obtained the bound

$$
\left\|T_{\mathfrak{m}_{a}}\right\|_{L_{p}\left(\mathbb{R}^{n}, \mathbb{R}\right) \rightarrow L_{p}\left(\mathbb{R}^{n}, \mathbb{R}\right)} \leqslant \max \left\{p, \frac{p}{p-1}\right\}-1 .
$$

Note that $\beta_{p}(\mathbb{C})=\max \{p, p /(p-1)\}-1$, by a theorem of Burkholder [16]. We are unable to recover the better estimate (83) for the scalar-valued case of Corollary 26 using our method.

Proof of Corollary 26. Write each $x \in \mathbb{R}^{n}$ as $x=\left(x_{1}, x^{\prime}\right)$, where $x^{\prime}=\left(x_{2}, \ldots, x_{n}\right) \in \mathbb{R}^{n-1}$. For every $s \in(0,1]$ define $\gamma_{s}: \mathbb{R}^{n} \rightarrow \mathbb{R}$ by

$$
\forall x \in \mathbb{R}^{n}, \quad \gamma_{s}(x) \stackrel{\text { def }}{=} \frac{x_{1}^{2}}{x_{1}^{2}+s\left\|x^{\prime}\right\|_{2}^{2}}=\int_{0}^{\infty} x_{1}^{2} e^{-\left(x_{1}^{2}+s\left\|x^{\prime}\right\|_{2}^{2}\right) t} \mathrm{~d} t .
$$

Then $\gamma_{1}=\mathfrak{m}_{2}$, and if $a \in(0,2)$ then by (80) with $\theta=a / 2 \in(0,1)$ and $\alpha=\left\|x^{\prime}\right\|_{2}^{2} / x_{1}^{2}$ we have

$$
\mathfrak{m}_{a}=\int_{0}^{1} \gamma_{s} d \mu_{a}(s)
$$

where $\mu_{a}$ is the probability measure on $(0,1)$ whose density is proportional to $s^{\frac{a}{2}-1}(1-s)^{-\frac{a}{2}}$. Therefore, in order to prove the desired estimate (82) it suffices to show that for every $s \in(0,1)$,

$$
\left\|T_{\gamma_{s}}\right\|_{L_{p}\left(\mathbb{R}^{n}, Y\right) \rightarrow L_{p}\left(\mathbb{R}^{n}, Y\right)} \leqslant \beta_{p}^{+}(Y)^{2} \beta_{p}^{-}(Y) .
$$

Consider the UMD Banach space $Z \stackrel{\text { def }}{=} L_{p}\left(\mathbb{R}^{n-1}, Y\right)$. By the identification $L_{p}\left(\mathbb{R}^{n}, Y\right) \cong L_{p}(\mathbb{R}, Z)$, the multiplier $T_{\gamma_{s}}$ can be thought of as an operator from $L_{p}(\mathbb{R}, Z)$ to $L_{p}(\mathbb{R}, Z)$; this is how Theorem 24 will be applied next, i.e., with $Y$ replaced by $Z$, while noting that $\beta_{p}^{ \pm}(Z)=\beta_{p}^{ \pm}(Y)$.

We consider a test function $f: \mathbb{R}^{n} \rightarrow Y$ that is finite linear combination of functions of the form $x \mapsto$ $f_{1}\left(x_{1}\right) f_{2}\left(x_{2}\right) \cdots f_{n}\left(x_{n}\right) y$, where $y \in Y$ and each $f_{i}$ has a smooth Fourier transform, compactly supported away from 0 , and a similar function $g^{*}: \mathbb{R}^{n} \rightarrow Y^{*}$. Note that such functions are dense in $L_{p}(\mathbb{R}, Z) \cong$ $L_{p}\left(\mathbb{R}^{n}, Y\right)$ and in $L_{q}\left(\mathbb{R}, Z^{*}\right)=L_{q}\left(\mathbb{R}, L_{q}\left(\mathbb{R}^{n-1}, Y^{*}\right)\right) \cong L_{q}\left(\mathbb{R}^{n}, Y^{*}\right)$, respectively, where $q=p /(p-1)$. Then by the Parseval identity (76) we have

$$
\int_{\mathbb{R}} g^{*}\left(x_{1}\right)\left(T_{\gamma_{s}} f\left(x_{1}\right)\right) \mathrm{d} x_{1}=\int_{\mathbb{R}^{n}} g^{*}(x)\left(T_{\gamma_{s}} f(x)\right) \mathrm{d} x=\int_{\mathbb{R}^{n}} \mathcal{F} g^{*}(x)\left(\frac{x_{1}^{2}}{x_{1}^{2}+s\left\|x^{\prime}\right\|_{2}^{2}} \mathcal{F} f(-x)\right) \mathrm{d} x .
$$


Consequently, by the second equality in (84),

$$
\begin{aligned}
\int_{\mathbb{R}} g^{*}\left(x_{1}\right)\left(T_{\gamma_{s}} f\left(x_{1}\right)\right) \mathrm{d} x_{1} & =\int_{0}^{\infty} \int_{\mathbb{R}^{n}} x_{1} e^{-\frac{t}{2} x_{1}^{2}} \mathcal{F} g^{*}(x)\left(e^{-t s\left\|x^{\prime}\right\|_{2}^{2}} x_{1} e^{-\frac{t}{2} x_{1}^{2}} \mathcal{F} f(-x)\right) \mathrm{d} x \mathrm{~d} t \\
& =\int_{0}^{\infty} \int_{\mathbb{R}^{n}} \mathcal{F}\left(\frac{\partial}{\partial x_{1}} e^{\frac{t}{2}\left(\frac{\partial}{\partial x_{1}}\right)^{2}} g^{*}\right)(x)\left(\mathcal{F}\left(-e^{t s \Delta^{\prime}} \frac{\partial}{\partial x_{1}} e^{\frac{t}{2}\left(\frac{\partial}{\partial x_{1}}\right)^{2}} f\right)\right)(-x) \mathrm{d} x \mathrm{~d} t
\end{aligned}
$$

where $\Delta^{\prime}$ denotes the Laplacian on $\mathbb{R}^{n-1}$, i.e., with respect to the variable $x^{\prime}$. By the vector-valued Parseval identity (75), we therefore have

$$
\int_{\mathbb{R}} g^{*}\left(x_{1}\right)\left(T_{\gamma_{s}} f\left(x_{1}\right)\right) \mathrm{d} x_{1}=\int_{0}^{\infty} \int_{\mathbb{R}}\left(\frac{\partial}{\partial x_{1}} e^{\frac{u}{2}\left(\frac{\partial}{\partial x_{1}}\right)^{2}} g^{*}\left(x_{1}\right)\right)\left(A(u) \frac{\partial}{\partial x_{1}} e^{\frac{u}{2}\left(\frac{\partial}{\partial x_{1}}\right)^{2}} f\right)\left(x_{1}\right) \mathrm{d} x_{1} \mathrm{~d} u,
$$

where $A(u) \stackrel{\text { def }}{=}-e^{u s \Delta^{\prime}}: L_{p}(\mathbb{R}, Z) \rightarrow L_{p}(\mathbb{R}, Z)$. Recalling Corollary 21 , it therefore follows from Theorem 24 that

$$
\begin{aligned}
& \left\|T_{\gamma_{s}}\right\|_{L_{p}\left(\mathbb{R}^{n}, Y\right) \rightarrow L_{p}\left(\mathbb{R}^{n}, Y\right)}=\left\|T_{\gamma_{s}}\right\|_{L_{p}(\mathbb{R}, Z) \rightarrow L_{p}(\mathbb{R}, Z)} \\
& \quad \leqslant \beta_{p}^{+}(Z) \beta_{p}^{-}(Z) \mathscr{R}_{p}\left(\left\{e^{t \Delta^{\prime}}: L_{p}\left(\mathbb{R}^{n-1}, Y\right) \rightarrow L_{p}\left(\mathbb{R}^{n-1}, Y\right)\right\}_{t \in(0, \infty)}\right) \leqslant \beta_{p}^{+}(Y)^{2} \beta_{p}^{-}(Y) .
\end{aligned}
$$

\subsection{Littlewood-Paley decomposition}

We need to introduce notation for the usual multi-scale bump functions that occur in Littlewood-Paley decompositions. Let $\phi: \mathbb{R} \rightarrow[0, \infty)$ be smooth and supported on $[-2,-1 / 2] \cup[1 / 2,2]$; for concreteness we can take

$$
\forall x \in \mathbb{R}, \quad \phi(x) \stackrel{\text { def }}{=} \begin{cases}e^{-\frac{1}{(|x|-1 / 2)(2-|x|)}} & \text { if }|x| \in(1 / 2,2), \\ 0 & \text { if }|x| \in[0,1 / 2] \cup[2, \infty) .\end{cases}
$$

For $k \in \mathbb{Z}$ define $\psi_{k}: \mathbb{R} \rightarrow \mathbb{R}$ by

$$
\forall x \in \mathbb{R}, \quad \psi_{k}(x) \stackrel{\text { def }}{=} \frac{\phi\left(2^{k} x\right)}{\sum_{j \in \mathbb{Z}} \phi\left(2^{j} x\right)} .
$$

We also define $\omega_{k}: \mathbb{R} \rightarrow \mathbb{R}$ by

$$
\forall x \in \mathbb{R}, \quad \omega_{k}(x) \stackrel{\text { def }}{=} \psi_{k-1}(x)+\psi_{k}(x)+\psi_{k+1}(x) .
$$

Thus $\omega_{k} \psi_{k}=\psi_{k}$, and therefore the corresponding multipliers satisfy the identity $T_{\omega_{k}} T_{\psi_{k}}=T_{\psi_{k}}$.

For every $k \in \mathbb{Z}$ define $\vartheta_{k}: \mathbb{R} \rightarrow[0, \infty)$ by

$$
\forall x \in \mathbb{R}, \quad \vartheta_{k}(x) \stackrel{\text { def }}{=} \frac{\sin ^{4}\left(2^{k} x\right)}{\left(2^{k} x\right)^{2}} .
$$

Like $\psi_{k}$, the function $\vartheta_{k}$ is roughly localized around $|x| \asymp 2^{-k}$, but unlike $\psi_{k}$, it has long tails that are supported over all of $\mathbb{R}$. The importance of the special "pseudo-bump functions" $\left\{\vartheta_{k}\right\}_{k \in \mathbb{Z}}$ stems from the fact that they can be directly related to averages of dyadic martingales, which leads to the following form of the Littlewood-Paley inequality with a good constant. 
Proposition 28. Suppose that $p \in(1, \infty)$ and let $\left(Y,\|\cdot\|_{Y}\right)$ be a UMD Banach space. Then

$$
\forall f \in L_{p}(\mathbb{R}, Y), \quad\left(\mathbb{E}_{\varepsilon \in\{-1,1\}^{\mathbb{Z}}}\left\|\sum_{j \in \mathbb{Z}} \varepsilon_{j} T_{\vartheta_{j}} f\right\|_{L_{p}(\mathbb{R}, Y)}^{p}\right)^{\frac{1}{p}} \leqslant \beta_{p}^{+}(Y)\|f\|_{L_{p}(\mathbb{R}, Y)} \leqslant \beta_{p}(Y)\|f\|_{L_{p}(\mathbb{R}, Y)} .
$$

Proposition 28 is due implicitly to Bourgain [12], where it is proved as an intermediate step towards a more usual form of the Littlewood-Paley inequality involving the localized bump functions $\left\{\psi_{k}\right\}_{k \in \mathbb{Z}}$ in place of $\left\{\vartheta_{k}\right\}_{k \in \mathbb{Z}}$, but with a more complicated dependence on the UMD constant. The above formulation of Proposition 28 appears explicitly as the special case $h=k=\mathbf{1}_{[0,1 / 2]}-\mathbf{1}_{[1 / 2,1]}$ of Proposition 5.10 in [40] (where we are using here the notation of [40, Proposition 5.10]).

In subsequent arguments we shall use Proposition 28 in addition to some auxiliary estimates concerning the bump functions $\left\{\psi_{k}\right\}_{k \in Z}$, which are valid for arbitrary Banach space targets. These estimates rely on the fact that if $\mathfrak{m} \in L_{1}(\mathbb{R})$ then by Young's inequality we have

$$
\forall f \in L_{p}(\mathbb{R}, Y), \quad\left\|T_{\mathfrak{m}} f\right\|_{L_{p}(\mathbb{R}, Y)}=\left\|\left(\mathcal{F}^{-1} \mathfrak{m}\right) * f\right\|_{L_{p}(\mathbb{R}, Y)} \leqslant\left\|\mathcal{F}^{-1} \mathfrak{m}\right\|_{L_{1}(\mathbb{R})}\|f\|_{L_{p}(\mathbb{R}, Y)} .
$$

Lemma 29. Suppose that $p \in[1, \infty]$ and that $\left(Y,\|\cdot\|_{Y}\right)$ is a Banach space. Then for every $k \in \mathbb{Z}$,

$$
\forall f \in L_{p}(\mathbb{R}, Y), \quad\left\|T_{\psi_{k}} f\right\|_{L_{p}(\mathbb{R}, Y)} \lesssim\left\|T_{\vartheta_{k}} f\right\|_{L_{p}(\mathbb{R}, Y)} .
$$

Proof. Since for every $x \in \mathbb{R}$ and $k \in \mathbb{Z}$ we have $\vartheta_{k}(x)=\vartheta_{0}\left(2^{k} x\right)$ and $\psi_{k}(x)=\psi_{0}\left(2^{k} x\right)$, we also have $T_{\vartheta_{k}} f(x)=T_{\vartheta_{0}}\left(y \mapsto f\left(2^{k} y\right)\right)\left(2^{-k} x\right)$ and $T_{\psi_{k}} f(x)=T_{\psi_{0}}\left(y \mapsto f\left(2^{k} y\right)\right)\left(2^{-k} x\right)$. Consequently, it suffices to prove (90) when $k=0$. Since $\vartheta_{0}$ is nonzero on the support of $\psi_{0}$, we can write $T_{\psi_{0}} f=T_{\psi_{0} / \vartheta_{0}} T_{\theta_{0}} f$. The function $\psi_{0} / \vartheta_{0}$ is smooth and compactly supported, therefore its inverse Fourier transform $g=$ $\mathcal{F}^{-1}\left(\psi_{0} / \vartheta_{0}\right)$ belongs to the Schwartz class of test functions $\mathscr{S}(\mathbb{R})$, and in particular $g \in L_{1}(\mathbb{R})$. Thus (90) follows from (89) with $\mathfrak{m}=\psi_{0} / \vartheta_{0}$ and $T_{\vartheta_{0}} f$ in place of $f$.

In order to facilitate the next two applications of (89), we record the following simple observation. If $\mathfrak{m} \in L_{1}(\mathbb{R})$ is smooth then for every $a \in(0, \infty)$ we have

$$
\begin{aligned}
\left\|\mathcal{F}^{-1} \mathfrak{m}\right\|_{L_{1}(\mathbb{R})} & \leqslant\left(\int_{\mathbb{R}} \frac{\mathrm{d} x}{1+(a x)^{2}}\right) \sup _{x \in \mathbb{R}}\left|\left(1+a^{2} x^{2}\right)\left(\mathcal{F}^{-1} \mathfrak{m}\right)(x)\right| \\
= & \frac{\pi}{a}\left\|\mathcal{F}^{-1}\left(\mathfrak{m}-a^{2} \mathfrak{m}^{\prime \prime}\right)\right\|_{L_{\infty}(\mathbb{R})} \leqslant \frac{\pi}{a}\left\|\mathfrak{m}-a^{2} \mathfrak{m}^{\prime \prime}\right\|_{L_{1}(\mathbb{R})} \leqslant \frac{\pi}{a}\|\mathfrak{m}\|_{L_{1}(\mathbb{R})}+\pi a\left\|\mathfrak{m}^{\prime \prime}\right\|_{L_{1}(\mathbb{R})} .
\end{aligned}
$$

Choosing $a=\sqrt{\|\mathfrak{m}\|_{L_{1}(\mathbb{R})} /\left\|\mathfrak{m}^{\prime \prime}\right\|_{L_{1}(\mathbb{R})}}$ in (91) and substituting the resulting estimate into (89) yields

$$
\forall f \in L_{p}(\mathbb{R}, Y), \quad\left\|T_{\mathfrak{m}} f\right\|_{L_{p}(\mathbb{R}, Y)} \leqslant 2 \pi \sqrt{\|\mathfrak{m}\|_{L_{1}(\mathbb{R})}\left\|\mathfrak{m}^{\prime \prime}\right\|_{L_{1}(\mathbb{R})}} \cdot\|f\|_{L_{p}(\mathbb{R}, Y)} .
$$

Lemma 30. Fix $k \in \mathbb{Z}$ and $p \in[1, \infty]$. Let $\left(Y,\|\cdot\|_{Y}\right)$ be a Banach space. Then for every $f \in L_{p}(\mathbb{R}, Y)$ and $y \in \mathbb{R}$ we have

$$
\left(\int_{\mathbb{R}}\left\|T_{\psi_{k}} f(x+y)-T_{\psi_{k}(x)} f(x)\right\|_{Y}^{p} \mathrm{~d} x\right)^{\frac{1}{p}} \lesssim \min \left\{1, \frac{|y|}{2^{k}}\right\}\left\|T_{\psi_{k}} f\right\|_{L_{p}(\mathbb{R}, Y)}
$$


Proof. Write $g(x) \stackrel{\text { def }}{=} T_{\psi_{k}} f(x+y)-T_{\psi_{k}} f(x)$. The fact that the norm of $g$ in $L_{p}(\mathbb{R}, Y)$ is at most twice the norm of $T_{\psi_{k}} f$ in $L_{p}(\mathbb{R}, Y)$ follows from the triangle inequality in $L_{p}(\mathbb{R}, Y)$. For the second estimate in (93), note that $g=T_{\rho_{y} \omega_{k}} T_{\psi_{k}} f$, where $\rho_{y}(x)=e^{-i x y}-1$. Hence, it remains to show that for every $h \in L_{p}(\mathbb{R}, Y)$ and $y \in\left[-2^{k}, 2^{k}\right]$ we have $\left\|T_{\rho_{y} \omega_{k}} h\right\|_{L_{p}(\mathbb{R}, Y)} \lesssim|y| 2^{-k}\|h\|_{L_{p}(\mathbb{R}, Y)}$. By (92), this will follow if we prove that $\left\|\rho_{y} \omega_{k}\right\|_{L_{1}(\mathbb{R})} \cdot\left\|\left(\rho_{y} \omega_{k}\right)^{\prime \prime}\right\|_{L_{1}(\mathbb{R})} \lesssim\left(2^{-k} y\right)^{2}$. Since $\rho_{y}(x) \omega_{k}(x)=\left(\rho_{2^{-k} y} \omega_{0}\right)\left(2^{k} y\right)$ and for every smooth $\mathfrak{m} \in L_{1}(\mathbb{R})$ the product $\|x \mapsto \mathfrak{m}(\lambda x)\|_{L_{1}(\mathbb{R})} \cdot\left\|(x \mapsto \mathfrak{m}(\lambda x))^{\prime \prime}\right\|_{L_{1}(\mathbb{R})}$ is independent of $\lambda \in(0, \infty)$, it suffices to show that

$$
\forall z \in[-1,1], \quad\left\|\rho_{z} \omega_{0}\right\|_{L_{1}(\mathbb{R})} \lesssim|z| \quad \text { and } \quad\left\|\left(\rho_{z} \omega_{0}\right)^{\prime \prime}\right\|_{L_{1}(\mathbb{R})} \lesssim|z| .
$$

The point-wise estimate $\left|\rho_{z}(x)\right| \leqslant|z x|$ combined with the fact that the function $x \mapsto x \omega_{0}(x)$ is in $L_{1}(\mathbb{R})$ implies the first assertion in (94). For the second assertion in (94), compute directly that

$$
\left(\rho_{z} \omega_{0}\right)^{\prime \prime}(x)=-z^{2} e^{-i x z} \omega_{0}(x)-i 2 z e^{-i x z} \omega_{0}^{\prime}(x)+\left(e^{-i x z}-1\right) \omega_{0}^{\prime \prime}(x) .
$$

We therefore have the following point-wise estimate, which holds true whenever $|z| \leqslant 1$.

$$
\left|\left(\rho_{z} \omega_{0}\right)^{\prime \prime}(x)\right| \leqslant|z|^{2}\left|\omega_{0}(x)\right|+2\left|z \omega_{0}^{\prime}(x)\right|+\left|z x \omega_{0}^{\prime \prime}(x)\right| \leqslant|z|\left(\left|\omega_{0}(x)\right|+2\left|\omega_{0}^{\prime}(x)\right|+\left|x \omega_{0}^{\prime \prime}(x)\right|\right) .
$$

The second assertion in (94) is now a consequence of the fact that the three functions $\omega_{0}, \omega_{0}^{\prime}$ and $x \mapsto x \omega_{0}^{\prime \prime}(x)$ all belong to $L_{1}(\mathbb{R})$. This concludes the proof of Lemma 30

Lemma 31. Fix $k \in \mathbb{Z}, p \in[1, \infty]$ and $\alpha \in(0, \infty)$. Let $\left(Y,\|\cdot\|_{Y}\right)$ be a Banach space. Then for every smooth and compactly supported $f: \mathbb{R} \rightarrow Y$ we have

$$
\left\|T_{\psi_{k}} f\right\|_{L_{p}(\mathbb{R}, Y)} \lesssim(1+\alpha) 2^{2 \alpha(k+2)}\left\|T_{\psi_{k}}(-\Delta)^{\alpha} f\right\|_{L_{p}(\mathbb{R}, Y)} .
$$

Proof. Recalling the definition of $\omega_{k}: \mathbb{R} \rightarrow[0, \infty)$ that is given in (87), since $T_{\omega_{k}} T_{\psi_{k}}=T_{\psi_{k}}$ we have $T_{\psi_{k}} f=(-\Delta)^{-\alpha} T_{\omega_{k}} T_{\psi_{k}}(-\Delta)^{\alpha} f=T_{\xi_{k}} T_{\psi_{k}}(-\Delta)^{\alpha} f$, where

$$
\forall x \in \mathbb{R} \backslash\{0\}, \quad \xi_{k}(x) \stackrel{\text { def }}{=} \frac{\omega_{k}(x)}{|x|^{2 \alpha}}=2^{2 k \alpha} \frac{\omega_{0}\left(2^{k} x\right)}{\left|2^{k} x\right|^{2 \alpha}}=2^{2 k \alpha} \xi_{0}\left(2^{k} x\right) .
$$

It therefore suffices to show that $\xi_{0}$ is the Fourier transform of a function in $L_{1}(\mathbb{R})$ of norm at most a constant multiple of $(1+\alpha) 2^{4 \alpha}$. To this end, we will again apply the estimate (92). First, since $|x|^{-1} \leqslant 4$ on the support of $\omega_{0}$ and $\omega_{0} \in L_{1}(\mathbb{R})$, we have $\left\|\xi_{0}\right\|_{L_{1}(\mathbb{R})} \lesssim 2^{4 \alpha}$. Also,

$$
\forall x \in \mathbb{R} \backslash\{0\}, \quad \xi_{0}^{\prime \prime}(x)=\frac{2 \alpha(2 \alpha+1)}{|x|^{2 \alpha}} \cdot \frac{\omega_{0}(x)}{x^{2}}-\frac{4 \alpha}{|x|^{2 \alpha}} \cdot \frac{\omega_{0}^{\prime}(x)}{|x|}+\frac{\omega_{0}^{\prime \prime}(x)}{|x|^{2 \alpha}} .
$$

Using again that $|x|^{-1} \leqslant 4$ on the support of $\omega_{0}$, combined with the fact that the three functions $\omega_{0}^{\prime \prime}$, $x \mapsto \omega_{0}(x) / x^{2}$ and $x \mapsto \omega_{0}^{\prime}(x) / x$ are all in $L_{1}(\mathbb{R})$, it follows that $\left\|\xi_{0}^{\prime \prime}\right\|_{L_{1}(\mathbb{R})} \lesssim(2 \alpha+1)^{2} 2^{4 \alpha}$. So,

$$
\left\|T_{\xi_{0}}\right\|_{L_{p}(\mathbb{R}, Y) \rightarrow L_{p}(\mathbb{R}, Y)} \stackrel{(92)}{\lesssim} \sqrt{\left\|\xi_{0}\right\|_{L_{1}(\mathbb{R})}\left\|\xi_{0}^{\prime \prime}\right\|_{L_{1}(\mathbb{R})}} \leqslant \sqrt{4^{2 \alpha} \cdot(2 \alpha+1)^{2} 4^{2 \alpha}} \lesssim(1+\alpha) 4^{2 \alpha} .
$$

Discrete ANALYsis, 2016:6, 48pp. 
QuAntitative AfFine ApProximation FOR UMD TARgEts

\subsection{Type and Cotype}

For $p \in[1,2)$ and $q \in[2, \infty)$, the type $p$ constant and the cotype $q$ constant of a Banach space $\left(Y,\|\cdot\|_{Y}\right)$, denoted $T_{p}(X)$ and $C_{q}(Y)$, respectively, are defined to be the infimum over those $T, C \in[1, \infty]$ such that for every $n \in \mathbb{N}$ and every $x_{1}, \ldots, x_{n} \in Y$,

$$
\frac{1}{C}\left(\sum_{j=1}^{n}\left\|x_{j}\right\|_{Y}^{q}\right)^{\frac{1}{q}} \leqslant \mathbb{E}\left[\left\|\sum_{j=1}^{n} \varepsilon_{j} x_{j}\right\|_{Y}\right] \leqslant T\left(\sum_{j=1}^{n}\left\|x_{j}\right\|_{Y}^{p}\right)^{\frac{1}{p}},
$$

where the expectation is with respect to $\varepsilon=\left(\varepsilon_{1}, \ldots, \varepsilon_{n}\right) \in\{-1,1\}^{n}$ chosen uniformly at random. The smallest $T, C \in(0, \infty]$ for which (96) holds true are denoted $T_{p}(Y), C_{q}(Y)$, respectively. Any UMD Banach space $\left(Y,\|\cdot\|_{Y}\right)$ admits an equivalent uniformly convex norm $[61,1]$, and hence it has finite cotype $[29,66]$. The following lemma makes this qualitative statement quantitative in the case of cotype. A similar (and simpler) argument yields a quantitative bound in the case of type as well (see Remark 33 below), but in what follows only the case of cotype will be used.

Lemma 32 (Cotype in terms of $\beta(Y)$ ). There exists a universal constant $\kappa \in(1, \infty)$ such that for every $\beta \in[1, \infty)$, if $\left(Y,\|\cdot\|_{Y}\right)$ is a UMD Banach space with $\beta(Y) \leqslant \beta$ then then $\left(Y,\|\cdot\|_{Y}\right)$ it has cotype $\kappa \beta$, and moreover $C_{\kappa \beta}(Y) \leqslant \kappa$.

Proof. The proof below is a (somewhat tedious) combination of several results that appear in the literature. The key step is an examination of the proof of Pisier's quantitative version [68] of the Maurey-Pisier theorem [63] for stable type. For $p \in(1,2)$ let $S T_{p}\left(Y^{*}\right)$ be the stable type $p$ constant of the dual space $Y^{*}$. Namely, $S T_{p}\left(Y^{*}\right)$ is the infimum over those $S \in(0, \infty]$ such that for every $n \in \mathbb{N}$, every $x_{1}^{*}, \ldots, x_{n}^{*} \in Y^{*}$ satisfy

$$
\mathbb{E}\left[\left\|\sum_{j=1}^{n} \theta_{j} x_{j}^{*}\right\|_{Y^{*}}\right] \leqslant S\left(\sum_{j=1}^{n}\left\|x_{j}^{*}\right\|_{Y^{*}}^{p}\right)^{\frac{1}{p}},
$$

where in (97) the expectation is with respect to i.i.d. standard symmetric $p$-stable random variables $\left\{\theta_{j}\right\}_{j=1}^{n}$, i.e., the characteristic function of $\theta_{1}$ is

$$
\forall t \in \mathbb{R}, \quad \mathbb{E}\left[e^{i t \theta_{1}}\right]=e^{-|t|^{p}} .
$$

It follows from (98) that $\mathbb{E}\left[\left|\theta_{1}\right|\right] \asymp 1 /(p-1)$; see e.g. [28, Sec. XVII]. By Jensen's inequality and Kahane's inequality, this implies that $T_{p}\left(Y^{*}\right) \leqslant(p-1) S T_{p}\left(Y^{*}\right)$. Since $C_{q}(Y) \leqslant T_{p}\left(Y^{*}\right)$, where $q=p /(p-1)$ (see e.g. $[62$, Sec. 6]), we deduce that

$$
S T_{p}\left(Y^{*}\right) \gtrsim \frac{C_{q}(Y)}{p-1} \asymp q C_{q}(Y) .
$$

Suppose from now on that $p \in(1,3 / 2]$ (in the argument below we only use that $p$ is bounded away from 2 by a universal constant). Equivalently, $q \in[3, \infty)$. It follows from the proof of the main theorem of [68] that there exists a universal constant $c \in(0,1)$ such that if $m \in \mathbb{N}$ satisfies

$$
m^{\frac{1}{q}} \leqslant \frac{c}{q} S T_{p}\left(Y^{*}\right) \leqslant c(p-1) S T_{p}\left(Y^{*}\right) .
$$




\section{TuOmas Hytönen, SEAn Li, AND AsSaf NaOR}

then $Y^{*}$ contains a 2-isomorphic copy of $\ell_{p}^{m}$. Unfortunately, while the dependence of $m$ on $S T_{p}\left(Y^{*}\right)$ that is stated in (100) is also stated explicitly in [68], the dependence on $p$, which is crucial for us here, is not computed in [68]. However, one can verify (100) by examining the dependencies on $p$ of certain constants that appear in [68], and substituting these dependencies into the proof of [68]. Specifically, the constant $C_{p}$ of Proposition 1.3 of [68] was computed in [58] to be

$$
C_{p}=\left(\frac{1}{\int_{0}^{\infty} \frac{\sin v}{v^{p}} d v}\right)^{\frac{1}{p}}=2\left(\frac{\Gamma\left(\frac{p+1}{2}\right)}{\sqrt{\pi} \Gamma\left(1-\frac{p}{2}\right)}\right)^{\frac{1}{p}}
$$

Thus, recalling that $p \in(1,3 / 2)$, we see that $C_{p}$ is bounded above and below by positive universal constants. The parameter $\Phi$ of Lemma 1.4 of [68] can be estimated via a direct computation (e.g., using the last line of page 975 of [31]) to give $\Phi \lesssim 1 /(p-1)$. The proof of [68] uses only two additional unspecified parameters, denoted $K$ and $\eta$, that appear in Lemma 1.5 of [68]. In Proposition 2 of [47] it is shown that one can take $K=2$ and $\eta=(2-p) /\left(8 p(q+1)^{q}\right)$. A direct substitution of these estimates into the proof of Theorem 2.1 of [68] now yields (100).

Note that

$$
\beta\left(\ell_{p}^{m}\right) \gtrsim \min \left\{q,(\log m)^{\frac{1}{p}}\right\}=\min \left\{q,(\log m)^{1-\frac{1}{q}}\right\} .
$$

While (101) is folklore, we did not find it in the literature so we briefly sketch the relevant computation. Let $k \in \mathbb{N}$ be the largest integer such that $2^{k} \leqslant m$. Let $\mu$ be the uniform probability measure on the discrete hypercube $\{-1,1\}^{k}$, and think of $\ell_{p}^{m}$ as containing an isometric copy of $L_{p}(\mu)$. Define $M_{0}, \ldots, M_{k}:\{-1,1\}^{k} \rightarrow L_{p}(\mu)$ by setting $M_{0} \equiv 1$ and for $j \in\{1, \ldots, k\}$ defining

$$
\forall \varepsilon, \delta \in\{-1,1\}^{k}, \quad M_{j}(\varepsilon)(\delta) \stackrel{\text { def }}{=} \prod_{\ell=1}^{k}\left(1+\varepsilon_{\ell} \boldsymbol{\delta}_{\ell}\right)=2^{k} \mathbf{1}_{\left\{\left(\varepsilon_{1}, \ldots, \varepsilon_{j}\right)=\left(\delta_{1}, \ldots, \delta_{j}\right)\right\}} .
$$

Then $\left\{M_{j}\right\}_{j=0}^{k}$ is a martingale with respect to the natural coordinate filtration of $\{-1,1\}^{k}$.

Observe that

$$
\left(\int_{\{-1,1\}^{k}}\left\|M_{k}(\varepsilon)\right\|_{L_{p}(\mu)}^{2} \mathrm{~d} \mu(\varepsilon)\right)^{\frac{1}{2}}=2^{\frac{k(p-1)}{p}}=2^{\frac{k}{q}} .
$$

For every $\varepsilon, \delta \in\{-1,1\}^{k}$ write $j(\varepsilon, \delta)=0$ if $\varepsilon_{1} \neq \delta_{1}$ and otherwise let $j(\varepsilon, \delta)$ be the largest $j \in\{1, \ldots, k\}$ such that $\varepsilon_{i}=\delta_{i}$ for all $i \in\{1, \ldots, j\}$. With this notation,

$$
S(\varepsilon, \delta) \stackrel{\text { def }}{=}\left(\sum_{j=1}^{k}\left(M_{j}(\varepsilon)(\delta)-M_{j-1}(\varepsilon)(\delta)\right)^{2}\right)^{\frac{1}{2}} \asymp 2^{j(\varepsilon, \delta)}-1 .
$$

Note that for every $j \in\{0, \ldots, k\}$ we have

$$
\mu \times \mu\left(\left\{(\varepsilon, \delta) \in\{-1,1\}^{k} \times\{-1,1\}^{k}: j(\varepsilon, \delta)=j\right\}\right) \asymp \frac{1}{2^{j}} .
$$


Hence, using the triangle inequality in $L_{p}(\mu)$ and Khinchine's inequality, we have

$$
\begin{aligned}
& \left(\int_{\{-1,1\}^{k}} \int_{\{-1,1\}^{k}}\left\|\sum_{j=1}^{k} \eta_{j}\left(M_{j}(\varepsilon)-M_{j-1}(\varepsilon)\right)\right\|_{L_{p}(\mu)}^{2} \mathrm{~d} \mu(\varepsilon) \mathrm{d} \mu(\eta)\right)^{\frac{1}{2}} \\
& \quad \gtrsim\left(\int_{\{-1,1\}^{k}} \int_{\{-1,1\}^{k}} S(\varepsilon, \delta)^{p} \mathrm{~d} \mu(\varepsilon) \mathrm{d} \mu(\delta)\right)^{\frac{1}{p}(103) \wedge(104)} \gtrsim\left(\sum_{j=1}^{k} 2^{j(p-1)}\right)^{\frac{1}{p}} \asymp 2^{\frac{k}{q}} \cdot \min \left\{q, k^{\frac{1}{p}}\right\},
\end{aligned}
$$

which, when contrasted with (102), implies (101).

A combination of (99), (100) and (101) implies that there exist universal $a, b \in(0,1 / 2)$ such that

$$
\forall q \in[3, \infty), \quad \beta(X) \geqslant a \min \left\{q,\left(q \log \left(1+b C_{q}(X)\right)\right)^{1-\frac{1}{q}}\right\} .
$$

For every $q \geqslant \beta(X) / a$ this gives $a\left(q \log \left(1+b C_{q}(X)\right)\right)^{2 / 3} \leqslant a\left(q \log \left(1+b C_{q}(X)\right)\right)^{1-1 / q} \leqslant \beta(X)$. Hence, $C_{q}(X) \leqslant e^{3 a /(2 e)} /\left(b a^{3 / 2}\right)$, since $q \geqslant \beta(X) / a$. By choosing $\kappa=\max \left\{1 / a, e^{3 a /(2 e)} /\left(b a^{3 / 2}\right)\right\}$, the proof of Lemma 32 is complete.

Remark 33. The same argument as in the proof of Lemma 32, without the need to use duality, shows that $T_{\kappa \beta /(\kappa \beta-1)}(Y) \leqslant \kappa$. Since we shall not need this fact below, the details are omitted.

\subsection{UMD-valued Riesz potentials, Sobolev spaces and interpolation}

Fix $n \in \mathbb{N}$ and $s, p \in(0, \infty)$. Suppose that $\left(Y,\|\cdot\|_{Y}\right)$ is a Banach space. If $f: \mathbb{R}^{n} \rightarrow Y$ is smooth and compactly supported then its homogeneous $(s, p)$-Riesz potential (semi)norm is defined as usual by

$$
\|f\|_{H_{s, p}\left(\mathbb{R}^{n}, Y\right)} \stackrel{\text { def }}{=}\left\|(-\Delta)^{\frac{s}{2}} f\right\|_{L_{p}\left(\mathbb{R}^{n}, Y\right)}=\left\|T_{\xi \mapsto\|\xi\|_{2}^{s}} f\right\|_{L_{p}\left(\mathbb{R}^{n}, Y\right)} .
$$

$\left(\|\cdot\|_{H_{s, p}\left(\mathbb{R}^{n}, Y\right)}\right.$ is sometimes denoted $\|\cdot\|_{\dot{H}_{s, p}\left(\mathbb{R}^{n}, Y\right)}$, but we use a simpler notation since nonhomogeneous Riesz potentials do not occur in what follows.) The Banach space $H_{s, p}\left(\mathbb{R}^{n}, Y\right)$ is the completion of the smooth and compactly support functions $f: \mathbb{R}^{n} \rightarrow Y$ under the norm $\|\cdot\|_{H_{s, p}\left(\mathbb{R}^{n}, Y\right)}$.

Throughout the ensuing discussion we shall use standard notation and basic facts from complex interpolation theory, as appearing in [9]. The following lemma provides quantitative control on the behavior of the spaces $H_{s, p}\left(\mathbb{R}^{n}, Y\right)$ under complex interpolation when $Y$ is a UMD Banach space.

Lemma 34. Let $\left(Y,\|\cdot\|_{Y}\right)$ be a UMD Banach space. Fix $p \in[1, \infty)$ and $s, \sigma \in(0, \infty)$ with $s<\sigma$. Suppose also that $\theta \in(0,1)$ and define $t=(1-\theta) s+\theta \sigma$. Then every $f \in H_{t, p}\left(\mathbb{R}^{n}, Y\right)$ satisfies

$$
\|f\|_{\left[H_{s, p}\left(\mathbb{R}^{n}, Y\right), H_{\sigma, p}\left(\mathbb{R}^{n}, Y\right)\right]_{\theta}} \lesssim \beta_{p}(Y) e^{\frac{\pi(\sigma-s)}{4} \sqrt{\theta(1-\theta)}}\|f\|_{H_{t, p}\left(\mathbb{R}^{n}, Y\right)} .
$$

Proof. Consider the strip $S \stackrel{\text { def }}{=}\{z \in \mathbb{C}: \mathfrak{R} z \in(0,1)\}$. For every $M \in(0, \infty)$ define an auxiliary mapping $\Phi_{M}: \bar{S} \rightarrow H_{s, p}\left(\mathbb{R}^{n}, Y\right)+H_{\sigma, p}\left(\mathbb{R}^{n}, Y\right)$ by

$$
\forall z \in \bar{S}, \quad \Phi_{M}(z) \stackrel{\text { def }}{=} e^{M(z(z-1)-\theta(\theta-1))}(-\Delta)^{\frac{t-s-z(\sigma-s)}{2}} f .
$$




\section{TuOmas Hytönen, SEAn Li, AND AsSaf NaOR}

Then $\Phi_{M}$ is holomorphic on $S$ and satisfies $\Phi_{M}(\theta)=f$. By the definition of the complex interpolation space $\left[H_{s, p}\left(\mathbb{R}^{n}, Y\right), H_{\sigma, p}\left(\mathbb{R}^{n}, Y\right)\right]_{\theta}$, we therefore have

$$
\|f\|_{\left[H_{s, p}\left(\mathbb{R}^{n}, Y\right), H_{\sigma, p}\left(\mathbb{R}^{n}, Y\right)\right]_{\theta}} \leqslant \inf _{M>0} \sup _{b \in \mathbb{R}} \max \left\{\left\|\Phi_{M}(i b)\right\|_{H_{s, p}\left(\mathbb{R}^{n}, Y\right)},\left\|\Phi_{M}(1+i b)\right\|_{H_{\sigma, p}\left(\mathbb{R}^{n}, Y\right)}\right\} .
$$

For every $(a, b) \in[0,1] \times \mathbb{R}$ we have

$$
(-\Delta)^{\frac{s+a(\sigma-s)}{2}} \Phi_{M}(a-b i)=e^{M a(a-1)+M \theta(1-\theta)-M b^{2}-M(2 a-1) b i}(-\Delta)^{\frac{i b(\sigma-s)}{2}}(-\Delta)^{\frac{t}{2}} f .
$$

Recalling (105), we therefore obtain the estimate

$$
\begin{aligned}
& \left\|\Phi_{M}(a+b i)\right\|_{H_{s+a(\sigma-s), p}\left(\mathbb{R}^{n}, Y\right)} \\
& \quad \leqslant e^{M a(a-1)+M \theta(1-\theta)-M b^{2}}\left\|(-\Delta)^{\frac{i b(\sigma-s)}{2}}\right\|_{L_{p}\left(\mathbb{R}^{n}, Y\right) \rightarrow L_{p}\left(\mathbb{R}^{n}, Y\right)}\|f\|_{H_{t, p}\left(\mathbb{R}^{n}, Y\right)} \\
& \quad \lesssim \beta_{p}(Y) e^{M a(a-1)+M \theta(1-\theta)} \cdot e^{-M b^{2}+\frac{\pi|b|(\sigma-s)}{4}}\|f\|_{H_{t, p}\left(\mathbb{R}^{n}, Y\right)},
\end{aligned}
$$

where in (107) we used Corollary 25. The function $b \mapsto-M b^{2}+\pi|b|(\sigma-s) / 4$ attains its maximum on $\mathbb{R}$ at $b=\pi(\sigma-s) /(8 M)$. It therefore follows from (107) that

$$
\sup _{b \in \mathbb{R}} \max \left\{\left\|\Phi_{M}(i b)\right\|_{H_{s, p}\left(\mathbb{R}^{n}, Y\right)},\left\|\Phi_{M}(1+i b)\right\|_{H_{\sigma, p}\left(\mathbb{R}^{n}, Y\right)}\right\} \lesssim \beta_{p}(Y) e^{M \theta(1-\theta)+\frac{\pi^{2}(\sigma-s)^{2}}{64 M}}\|f\|_{H_{t, p}\left(\mathbb{R}^{n}, Y\right)}
$$

In combination with (106) we therefore have

$$
\begin{aligned}
\|f\|_{\left[H_{s, p}\left(\mathbb{R}^{n}, Y\right), H_{\sigma, p}\left(\mathbb{R}^{n}, Y\right)\right]_{\theta}} & \lesssim \beta_{p}(Y)\left(\inf _{M \in(0, \infty)} e^{M \theta(1-\theta)+\frac{\pi^{2}(\sigma-s)^{2}}{64 M}}\right)\|f\|_{H_{t, p}\left(\mathbb{R}^{n}, Y\right)} \\
& =\beta_{p}(Y) e^{\frac{\pi(\sigma-s)}{4} \sqrt{\theta(1-\theta)}}\|f\|_{H_{t, p}\left(\mathbb{R}^{n}, Y\right)},
\end{aligned}
$$

where for (108) the optimal choice of $M \in(0, \infty)$ is $M=\pi(\sigma-s) /(8 \sqrt{\theta(1-\theta)})$.

Suppose that $\Omega \subseteq \mathbb{R}^{n}$ is open (for our purposes $\Omega$ will always be either a multiple of $B^{n}$ or all of $\mathbb{R}^{n}$ ). If $\left(Y,\|\cdot\|_{Y}\right)$ is a Banach space and $p \in[1, \infty]$ then for every smooth $f: \Omega \rightarrow Y$ denote

$$
\|f\|_{W_{1, p}(\Omega, Y)} \stackrel{\text { def }}{=} \sum_{j=1}^{n}\left\|\frac{\partial f}{\partial x_{j}}\right\|_{L_{p}(\Omega, Y)} .
$$

Thus $\|\cdot\|_{W_{p, 1}(\Omega, Y)}$ is the (homogeneous) first order Sobolev (semi)norm of $f$. The corresponding Sobolev space $W_{p, 1}(\Omega, Y)$ is the completion of the space of all smooth and compactly supported functions $f: \mathbb{R}^{n} \rightarrow Y$ under the norm $\|\cdot\|_{W_{p, 1}(\Omega, Y)}$. For $(s, p) \in(0,1) \times[1, \infty)$, the order $s$ fractional (homogeneous) Sobolev (semi)norm of $f: \Omega \rightarrow Y$ is defined by

$$
\|f\|_{W_{s, p}(\Omega, Y)} \stackrel{\text { def }}{=}\left(\iint_{\Omega \times \Omega} \frac{\|f(x)-f(y)\|_{Y}^{p}}{\|x-y\|_{2}^{n+p s}} \mathrm{~d} x \mathrm{~d} y\right)^{\frac{1}{p}} .
$$


While the notation $\|\cdot\|_{\dot{W}_{s, p}(\Omega, Y)}$ is sometimes used in the literature, we shall use the above simpler notation because nonhomogeneous Sobolev norms do not occur in what follows.

Our next goal is to relate UMD-valued Riesz potentials to Sobolev norms. The following lemma treats the case of first order Sobolev norms, and also contains a comparison between Riesz potentials that will be needed later; it is a simple consequence of the boundedness of the Hilbert transform on UMD Banach spaces, combined with the method of rotations.

Lemma 35. Suppose that $\left(Y,\|\cdot\|_{Y}\right)$ is a UMD Banach space, $n \in \mathbb{N}$ and $p \in(1, \infty)$. Then every $f \in W_{1, p}\left(\mathbb{R}^{n}, Y\right)$ satisfies

$$
\|f\|_{H_{1, p}\left(\mathbb{R}^{n}, Y\right)} \leqslant \beta_{p}(Y)^{2}\|f\|_{W_{1, p}\left(\mathbb{R}^{n}, Y\right)} .
$$

Moreover, if $s \in(1, \infty)$ and $j \in\{1, \ldots, n\}$ then for every smooth $f: \mathbb{R}^{n} \rightarrow Y$ we have

$$
\left\|\frac{\partial f}{\partial x_{j}}\right\|_{H_{s-1, p}\left(\mathbb{R}^{n}, Y\right)} \leqslant \beta_{p}(Y)^{2}\|f\|_{H_{s, p}\left(\mathbb{R}^{n}, Y\right)} .
$$

Proof. Suppose that $K: \mathbb{R}^{n} \rightarrow Y$ is odd, continuous on $\mathbb{R}^{n} \backslash\{0\}$, and positively homogeneous of order $-n$, i.e., $K(t x)=t^{-n} K(x)$ for every $t \in(0, \infty)$ and $x \in \mathbb{R}^{n} \backslash\{0\}$. For $f \in L_{p}\left(\mathbb{R}^{n}, X\right)$ consider the corresponding Calderòn-Zygmund singular integral

$$
\forall x \in \mathbb{R}^{n}, \quad T_{K} f(x) \stackrel{\text { def }}{=} \int_{\mathbb{R}^{n}} K(x-y) f(y) \mathrm{d} y .
$$

It follows from the method of rotations, as presented by Iwaniec and Martin in [44], that

$$
\left\|T_{K}\right\|_{L_{p}\left(\mathbb{R}^{n}, Y\right) \rightarrow L_{p}\left(\mathbb{R}^{n}, Y\right)} \leqslant \frac{\pi}{2}\left(\int_{S^{n-1}}|K(z)| \mathrm{d} \sigma(z)\right)\|H\|_{L_{p}(\mathbb{R}, Y) \rightarrow L_{p}(\mathbb{R}, Y)},
$$

where $\sigma$ is the surface area measure on the Euclidean sphere $S^{n-1}$ and $H: L_{p}(\mathbb{R}, Y) \rightarrow L_{p}(\mathbb{R}, Y)$ is the Hilbert transform, i.e.,

$$
\forall \varphi \in L_{p}(\mathbb{R}, Y), \quad H \varphi(x) \stackrel{\text { def }}{=} \frac{1}{\pi} \int_{\mathbb{R}} \frac{\varphi(y)}{x-y} \mathrm{~d} y .
$$

The integrals in (112) and (114) exist in the sense of principal values. The estimate (113) is presented in [44, Proposition 5.1] in the case $Y=\mathbb{C}$, but the same proof applies to the Banach space-valued setting without any change (the proof is based on an integral identity that is estimated using convexity of the norm. As such, the vector-valued and scalar-valued cases are identical; here the Banach space $Y$ can be general and the UMD property isn't used).

For $j \in\{1, \ldots, n\}$ consider the Riesz transform given by

$$
\forall x \in \mathbb{R}^{n}, \quad R_{j} f(x) \stackrel{\text { def }}{=} \frac{\Gamma\left(\frac{n+1}{2}\right)}{\pi^{\frac{n+1}{2}}} \int_{\mathbb{R}^{n}} \frac{x_{j}-y_{j}}{\|x-y\|_{2}^{n+1}} f(y) \mathrm{d} y .
$$

By (113) we have,

$$
\begin{aligned}
\left\|R_{j}\right\|_{L_{p}\left(\mathbb{R}^{n}, Y\right) \rightarrow L_{p}\left(\mathbb{R}^{n}, Y\right)} & \leqslant \frac{\pi \Gamma\left(\frac{n+1}{2}\right) \int_{S^{n-1}}\left|z_{1}\right| \mathrm{d} \sigma(z)}{2 \pi^{\frac{n+1}{2}}}\|H\|_{L_{p}(\mathbb{R}, Y) \rightarrow L_{p}(\mathbb{R}, Y)} \\
& =\|H\|_{L_{p}(\mathbb{R}, Y) \rightarrow L_{p}(\mathbb{R}, Y)} \leqslant \beta_{p}(Y)^{2}
\end{aligned}
$$




\section{Tuomas Hytönen, SeAn Li, And Assaf NaOR}

where the bound $\|H\|_{L_{p}(\mathbb{R}, Y) \rightarrow L_{p}(\mathbb{R}, Y)} \leqslant \beta_{p}(Y)^{2}$ that was used in (115) is implicit in the important work of Burkholder [15] and explicit in [32, Theorem 3].

Note that for every $j \in\{1, \ldots, n\}$ we have $R_{j}=(-\Delta)^{-1 / 2} \frac{\partial}{\partial x_{j}}$, as follows directly by computing the Fourier transform (see e.g. [72, Chapter III])). Consequently,

$$
\|f\|_{H_{1, p}\left(\mathbb{R}^{n}, Y\right)} \stackrel{(105)}{=}\left\|(-\Delta)^{-\frac{1}{2}} \Delta f\right\|_{L_{p}\left(\mathbb{R}^{n}, Y\right)}=\left\|\sum_{j=1}^{n} R_{j} \frac{\partial f}{\partial x_{j}}\right\|_{L_{p}\left(\mathbb{R}^{n}, Y\right)} \stackrel{(109) \wedge(115)}{\lessgtr} \beta_{p}(Y)^{2}\|f\|_{W_{1, p}\left(\mathbb{R}^{n}, Y\right)} .
$$

Finally, to deduce (111), proceed as follows.

$$
\begin{array}{r}
\left\|\frac{\partial f}{\partial x_{j}}\right\|_{H_{s-1, p}\left(\mathbb{R}^{n}, Y\right)} \stackrel{(105)}{=}\left\|(-\Delta)^{\frac{s-1}{2}} \frac{\partial f}{\partial x_{j}}\right\|_{L_{p}\left(\mathbb{R}^{n}, Y\right)}=\left\|(-\Delta)^{-\frac{1}{2}} \frac{\partial}{\partial x_{j}}(-\Delta)^{\frac{s}{2}} f\right\|_{L_{p}\left(\mathbb{R}^{n}, Y\right)} \\
=\left\|R_{j}(-\Delta)^{\frac{s}{2}} f\right\|_{L_{p}\left(\mathbb{R}^{n}, Y\right)} \stackrel{(115)}{\leqslant} \beta_{p}(Y)^{2}\left\|(-\Delta)^{\frac{s}{2}} f\right\|_{L_{p}\left(\mathbb{R}^{n}, Y\right)} \stackrel{(105)}{=} \beta_{p}(Y)^{2}\|f\|_{H_{s, p}\left(\mathbb{R}^{n}, Y\right)} .
\end{array}
$$

The following theorem asserts a useful comparison between UMD-valued fractional Sobolev norms and the corresponding Riesz potentials.

Theorem 36. Fix $n \in \mathbb{N}, s \in(0,1)$ and $p \in[2, \infty)$. Suppose that $\left(Y,\|\cdot\|_{Y}\right)$ is a UMD Banach space of cotype $p$. Then every $f \in H_{s, p}\left(\mathbb{R}^{n}, Y\right)$ satisfies

$$
\|f\|_{W_{s, p}\left(\mathbb{R}^{n}, Y\right)} \lesssim \frac{C_{p}(Y) \beta_{p}(Y)^{4}\left(n V_{n}\right)^{\frac{1}{p}}}{s(1-s)}\|f\|_{H_{s, p}\left(\mathbb{R}^{n}, Y\right)},
$$

Proof of Theorem 36. The proof below proceeds via a reduction to a slightly stronger statement in the one dimensional case $n=1$. So, assume for the moment that we already proved that

$$
\forall g \in H_{s, p}(\mathbb{R}, Y), \quad\|g\|_{W_{s, p}(\mathbb{R}, Y)} \lesssim \frac{C_{p}(Y) \beta_{p}(Y)}{s(1-s)}\|g\|_{H_{s, p}(\mathbb{R}, Y)}
$$

We shall now deduce the desired estimate (116) from (117), and then proceed to prove (117).

For every $z \in S^{n-1}$ and $w \in z^{\perp} \subseteq \mathbb{R}^{n}$ define $g_{z, w}: \mathbb{R} \rightarrow Y$ by setting

$$
\forall t \in \mathbb{R}, \quad g_{z, w}(t) \stackrel{\text { def }}{=} f(w+t z) .
$$

By changing to polar coordinates we see that

$$
\begin{aligned}
\|f\|_{W_{s, p}\left(\mathbb{R}^{n}, Y\right)}^{p} & \stackrel{(110)}{=} \quad \iint_{\mathbb{R}^{n} \times \mathbb{R}^{n}} \frac{\|f(x+y)-f(x)\|_{Y}^{p}}{\|y\|_{2}^{n+p s}} \mathrm{~d} x \mathrm{~d} y \\
= & \frac{1}{2} \iiint_{S^{n-1} \times \mathbb{R}^{n} \times \mathbb{R}} \frac{\|f(x+r z)-f(x)\|_{Y}^{p}}{|r|^{1+p s}} \mathrm{~d} \sigma(z) \mathrm{d} x \mathrm{~d} r \\
\stackrel{(110) \wedge(118)}{=} & \frac{1}{2} \int_{S^{n-1}} \mathrm{~d} \sigma(z) \int_{z^{\perp}}\left\|g_{z, w}\right\|_{W_{s, p}(\mathbb{R}, Y)}^{p} \mathrm{~d} w .
\end{aligned}
$$


Hence, using (117) we deduce that

$$
\|f\|_{W_{s, p}\left(\mathbb{R}^{n}, Y\right)} \lesssim \frac{C_{p}(Y) \beta_{p}(Y)}{s(1-s)}\left(\int_{S^{n-1}} \mathrm{~d} \sigma(z) \int_{z^{\perp}}\left\|g_{z, w}\right\|_{H_{s, p}(\mathbb{R}, Y)}^{p} \mathrm{~d} w\right)^{\frac{1}{p}}
$$

For every $z \in S^{n-1}, w \in z^{\perp}$ and $t \in \mathbb{R}$ denote

$$
h_{z, w}(t) \stackrel{\text { def }}{=}\left(-\langle z, \nabla\rangle^{2}\right)^{\frac{s}{2}} f(w+t z) \stackrel{(118)}{=}\left(-\frac{\partial^{2}}{\partial t^{2}}\right)^{\frac{s}{2}} g_{z, w}(t) .
$$

With this notation, for every $z \in S^{n-1}$ we have

$$
\begin{aligned}
\int_{z^{\perp}}\left\|g_{z, w}\right\|_{H_{s, p}(\mathbb{R}, Y)}^{p} \mathrm{~d} w \stackrel{(105)}{=} \int_{z^{\perp}}\left\|h_{z, w}\right\|_{L_{p}(\mathbb{R}, Y)}^{p} \mathrm{~d} w=\left\|\left(-\langle z, \nabla\rangle^{2}\right)^{\frac{s}{2}} f\right\|_{L_{p}\left(\mathbb{R}^{n}, Y\right)}^{p} \\
=\left\|\left(-\langle z, \nabla\rangle^{2}\right)^{\frac{s}{2}}(-\Delta)^{-\frac{s}{2}}(-\Delta)^{\frac{s}{2}} f\right\|_{L_{p}\left(\mathbb{R}^{n}, Y\right)}^{p} \stackrel{(105)}{\leqslant}\left\|T_{m_{z}}\right\|_{L_{p}\left(\mathbb{R}^{n}, Y\right) \rightarrow L_{p}\left(\mathbb{R}^{n}, Y\right)}^{p}\|f\|_{H_{s, p}\left(\mathbb{R}^{n}, Y\right)}^{p},
\end{aligned}
$$

where we set $m_{z}(x)=|\langle z, x\rangle|^{s} /\|x\|_{2}^{s}$ for $x \in \mathbb{R}^{n}$ and $z \in S^{n-1}$. By rotation invariance, it follows from Corollary 26 that the norm of the multiplier $T_{m_{z}}$ as an operator from $L_{p}\left(\mathbb{R}^{n}, Y\right)$ to itself is at most a constant multiple of $\beta_{p}(Y)^{3}$. By combining this bound with (119) and (120), we get

$$
\|f\|_{W_{s, p}\left(\mathbb{R}^{n}, Y\right)} \lesssim \frac{C_{p}(Y) \beta_{p}(Y)^{4} \sigma\left(S^{n-1}\right)^{\frac{1}{p}}}{s(1-s)}\|f\|_{H_{s, p}\left(\mathbb{R}^{n}, Y\right)}
$$

Since $\sigma\left(S^{n-1}\right)=n V_{n}$, this concludes the deduction of (116) from (117).

It remains to prove (117). The case $Y=\mathbb{R}$ of (117) is given in [9, Theorem 6.2.5], without explicit dependence on the relevant parameters. The proof of (117) below consists of an adaptation of the argument of [9, Theorem 6.2.5] to the UMD-valued setting, while tracking the bounds.

Recalling the Littlewood-Paley partition of unity $\left\{\psi_{j}\right\}_{j \in \mathbb{Z}}$ given in (86), for every $y \in \mathbb{R}$ we have

$$
\begin{aligned}
\left(\int_{\mathbb{R}}\|g(x+y)-g(x)\|_{Y}^{p} \mathrm{~d} x\right)^{\frac{1}{p}} & \leqslant \sum_{j \in \mathbb{Z}}\left(\int_{\mathbb{R}}\left\|T_{\psi_{j}} g(x+y)-T_{\psi_{j}(x)} g(x)\right\|_{Y}^{p} \mathrm{~d} x\right)^{\frac{1}{p}} \\
& \lesssim \sum_{j \in \mathbb{Z}} \min \left\{1, \frac{|y|}{2^{j}}\right\}\left\|T_{\psi_{j} g}\right\|_{L_{p}(\mathbb{R}, Y)},
\end{aligned}
$$

where in (121) we used the fact that $\sum_{j \in \mathbb{Z}} \psi_{j} \equiv 1$ and the triangle inequality in $L_{p}(\mathbb{R}, Y)$, and in (122) we 
used Lemma 30. Now,

$$
\begin{aligned}
&\|g\|_{W_{s, p}(\mathbb{R}, Y)} \stackrel{(122)}{\lesssim}\left(\sum_{r \in \mathbb{Z}} \int_{2^{r}}^{2^{r+1}}\left(\sum_{j \in \mathbb{Z}} \min \left\{1, \frac{|y|}{2^{j}}\right\}\left\|T_{\psi_{j}} g\right\|_{L_{p}(\mathbb{R}, Y)}\right)^{p} \frac{\mathrm{d} y}{|y|^{1+p s}}\right)^{\frac{1}{p}} \\
& \asymp\left(\sum_{r \in \mathbb{Z}}\left(\sum_{u \in \mathbb{Z}} \frac{\min \left\{1,2^{u}\right\}}{2^{s u}} \cdot 2^{-s(r-u)}\left\|T_{\psi_{r-u}} g\right\|_{L_{p}(\mathbb{R}, Y)}\right)^{p}\right)^{\frac{1}{p}} \\
& \leqslant \sum_{u \in \mathbb{Z}}\left(\sum_{r \in \mathbb{Z}} \frac{\min \left\{1,2^{p u}\right\}}{2^{p s u}} \cdot 2^{-p s(r-u)}\left\|T_{\psi_{r-u}} g\right\|_{L_{p}(\mathbb{R}, Y)}^{p}\right)^{\frac{1}{p}} \\
&=\left(\sum_{j \in \mathbb{Z}} 2^{-p s j}\left\|T_{\psi_{j}} g\right\|_{L_{p}(\mathbb{R}, Y)}^{p}\right)^{\frac{1}{p}} \sum_{u \in \mathbb{Z}} \frac{\min \left\{1,2^{u}\right\}}{2^{s u}} \\
& \asymp \frac{1}{s(1-s)}\left(\sum_{j \in \mathbb{Z}} 2^{-p s j}\left\|T_{\psi_{j}} g\right\|_{L_{p}(\mathbb{R}, Y)}^{p}\right)^{\frac{1}{p}}
\end{aligned}
$$

where for (124) use the fact that for each $r \in \mathbb{Z}$ in the integrand of the corresponding summand that appears in the right hand side of (123) we have $|y| \asymp 2^{r}$, and make the change of variable $u=r-j$, and for (125) use the triangle inequality in $\ell_{p}(\mathbb{Z})$.

Recalling the functions $\left\{\vartheta_{j}\right\}_{j \in \mathbb{Z}}$ given in (88), an application of Proposition 28 shows that

$$
\mathbb{E}_{\varepsilon \in\{-1,1\}^{\mathbb{Z}}}\left[\left\|\sum_{j \in \mathbb{Z}} \varepsilon_{j} T_{\vartheta_{j}}(-\Delta)^{\frac{s}{2}} g\right\|_{L_{p}(\mathbb{R}, Y)}\right] \leqslant \beta_{p}(Y)\left\|(-\Delta)^{\frac{s}{2}} g\right\|_{L_{p}(\mathbb{R}, Y)}=\beta_{p}(Y)\|g\|_{H_{s, p}(\mathbb{R}, Y)}
$$

At the same time, since $L_{p}(\mathbb{R}, Y)$ has cotype $p$ with constant $C_{p}(Y)$,

$$
\begin{aligned}
\mathbb{E}_{\varepsilon \in\{-1,1\}^{\mathbb{Z}}}\left[\left\|\sum_{j \in \mathbb{Z}} \varepsilon_{j} T_{\vartheta_{j}}(-\Delta)^{\frac{s}{2}} g\right\|_{L_{p}(\mathbb{R}, Y)}\right] & \gtrsim \frac{1}{C_{p}(Y)}\left(\sum_{j \in \mathbb{Z}}\left\|T_{\vartheta_{j}}(-\Delta)^{\frac{s}{2}} g\right\|_{L_{p}(\mathbb{R}, Y)}^{p}\right)^{\frac{1}{p}} \\
& \gtrsim \frac{1}{C_{p}(Y)}\left(\sum_{j \in \mathbb{Z}}\left\|T_{\psi_{j}}(-\Delta)^{\frac{s}{2}} g\right\|_{L_{p}(\mathbb{R}, Y)}^{p}\right)^{\frac{1}{p}} \gtrsim \frac{1}{C_{p}(Y)}\left(\sum_{j \in \mathbb{Z}} 2^{-p s j}\left\|T_{\psi_{j}} g\right\|_{L_{p}(\mathbb{R}, Y)}^{p}\right)^{\frac{1}{p}},
\end{aligned}
$$

where in the penultimate step of (128) we used Lemma 90 and in the final step of (128) we used Lemma 31. By combining (127) and (128), we see that

$$
\left(\sum_{j \in \mathbb{Z}} 2^{-p s j}\left\|T_{\psi_{j}} g\right\|_{L_{p}(\mathbb{R}, Y)}^{p}\right)^{\frac{1}{p}} \lesssim C_{p}(Y) \beta_{p}(Y)\|g\|_{H_{s, p}(\mathbb{R}, Y)} .
$$

A substitution of (129) into (126) now yields the desired estimate (117).

Remark 37. The left side of (129) is the Besov norm $\|g\|_{B_{p, p}^{s}(\mathbb{R}, Y)}$. Hence (129) asserts a quantitative embedding of $H_{s, p}(\mathbb{R}, Y)$ into $B_{p, p}^{s}(\mathbb{R}, Y)$ when $Y$ is a UMD space of cotype $p$. A qualitative embedding statement of this type was recently established by Veraar [79, Proposition 3.1]. 
Remark 38. One can prove a reverse inequality to that of Theorem 36 under the assumption that $\left(Y,\|\cdot\|_{Y}\right)$ is a UMD Banach space of type $p \in(1,2]$, in which case one obtains an upper bound on $\|f\|_{H_{s, p}\left(\mathbb{R}^{n}, Y\right)}$ in terms of $\|f\|_{W_{s, p}\left(\mathbb{R}^{n}, Y\right)}$. Specifically, we have the following estimate for $s>0$.

$$
\forall f \in W_{s, p}\left(\mathbb{R}^{n}, Y\right), \quad\|f\|_{H_{s, p}\left(\mathbb{R}^{n}, Y\right)} \lesssim \frac{T_{p}(Y) \beta_{p}(Y)^{4} n^{\frac{s}{2}}}{(p-1)\left(n V_{n}\right)^{1-\frac{1}{p}}}\|f\|_{W_{s, p}\left(\mathbb{R}^{n}, Y\right)} .
$$

Since (130) is not needed below, we omit its proof (which is available on request). By Remark 33, one can take in (130) $p-1 \asymp 1 / \beta(Y)$, in which case $T_{p}(Y) \asymp 1$ and, by (49), $\beta_{p}(Y) \lesssim \beta(Y)^{2}$. Note that, by Kwapien's theorem [52], unless $Y$ is isomorphic to a Hilbert space, its type and cotype do not coincide, and therefore by combining Theorem 36 with (130) one does not obtain an equivalence between the norms $\|\cdot\|_{H_{s, p}\left(\mathbb{R}^{n}, Y\right)}$ and $\|\cdot\|_{W_{s, p}\left(\mathbb{R}^{n}, Y\right)}$ for non-Hilbertian targets $Y$. See [38] for a related characterization of Hilbert space.

Recalling the notation that was introduced in Section 2, we end this section by deducing a corollary of Theorem 36 that will be very important in what follows.

Corollary 39. Fix $p \in[2, \infty)$ and $(s, \sigma) \in(0,1) \times(1,2)$. Let $\left(Y,\|\cdot\|_{Y}\right)$ be a UMD Banach space of cotype $p$. Then every measurable $f: \mathbb{R}^{n} \rightarrow Y$ satisfies

$$
\left(\iiint_{\mathbb{R}^{n} \times B^{n} \times(0, \infty)} \frac{\left\|f^{x}(u y)-P_{u}^{1} f^{x}(u y)\right\|_{Y}^{p}}{V_{n} u^{p s+1}} \mathrm{~d} x \mathrm{~d} y \mathrm{~d} u\right)^{\frac{1}{p}} \lesssim \frac{\sqrt{p n} C_{p}(Y) \beta_{p}(Y)^{4}}{s(1-s)}\|f\|_{H_{s, p}\left(\mathbb{R}^{n}, Y\right)},
$$

and

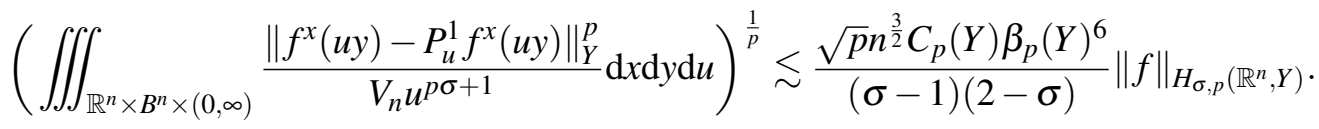

Proof. By integrating the conclusion of Corollary 14 (with $q=p s$ ) over $x \in \mathbb{R}^{n}$ we see that

$$
\left(\frac{1}{V_{n}} \iiint_{\mathbb{R}^{n} \times B^{n} \times(0, \infty)} \frac{\left\|f^{x}(u y)-P_{u}^{1} f^{x}(u y)\right\|_{Y}^{p}}{u^{p s+1}} \mathrm{~d} x \mathrm{~d} y \mathrm{~d} u\right)^{\frac{1}{p}} \lesssim \frac{\sqrt{p n}}{\left(n V_{n}\right)^{\frac{1}{p}}}\|f\|_{W_{s, p}\left(\mathbb{R}^{n}, Y\right)} .
$$

A substitution of the conclusion of Theorem 36 into (133) yields (131).

Next, by integrating the conclusion of Corollary 15 (with $q=p \sigma>p$ ) over $x \in \mathbb{R}^{n}$ we see that

$$
\left(\frac{1}{V_{n}} \iiint_{\mathbb{R}^{n} \times B^{n} \times(0, \infty)} \frac{\left\|f^{x}(u y)-P_{u}^{1} f^{x}(u y)\right\|_{Y}^{p}}{u^{p \sigma+1}} \mathrm{~d} x \mathrm{~d} y \mathrm{~d} u\right)^{\frac{1}{p}} \lesssim \frac{\sqrt{p n}}{\left(n V_{n}\right)^{\frac{1}{p}}} \sum_{j=1}^{n}\left\|\frac{\partial f}{\partial x_{j}}\right\|_{W_{\sigma-1, p}\left(\mathbb{R}^{n}, Y\right)} .
$$

By Theorem 36 applied to $\frac{\partial f}{\partial x_{j}}$ for each $j \in\{1, \ldots, n\}$ and with $s$ replaced by $\sigma-1 \in(0,1)$,

$$
\forall j \in\{1, \ldots, n\}, \quad\left\|\frac{\partial f}{\partial x_{j}}\right\|_{W_{\sigma-1, p}\left(\mathbb{R}^{n}, Y\right)} \lesssim \frac{C_{p}(Y) \beta_{p}(Y)^{4}\left(n V_{n}\right)^{\frac{1}{p}}}{(\sigma-1)(2-\sigma)}\left\|\frac{\partial f}{\partial x_{j}}\right\|_{H_{\sigma-1, p}\left(\mathbb{R}^{n}, Y\right)} .
$$


Moreover, by the second assertion of Lemma 35 we have

$$
\max _{j \in\{1, \ldots, n\}}\left\|\frac{\partial f}{\partial x_{j}}\right\|_{H_{\sigma-1, p}\left(\mathbb{R}^{n}, Y\right)} \lesssim \beta_{p}^{2}(Y)\|f\|_{H_{\sigma, p}\left(\mathbb{R}^{n}, Y\right)} .
$$

By substituting (136) into (135), and then substituting the resulting estimate into (134), we obtain the desired estimate (132).

\section{Proof of Theorem 19}

We shall prove here the following theorem.

Theorem 40. Fix $p \in[2, \infty)$ and suppose that $\left(Y,\|\cdot\|_{Y}\right)$ is a UMD Banach space of cotype $p$. Then every smooth $f: \mathbb{R}^{n} \rightarrow Y$ satisfies

$$
\begin{aligned}
\left(\iiint_{\mathbb{R}^{n} \times B^{n} \times(0, \infty)} \frac{\left\|f^{x}(u y)-P_{u}^{1} f^{x}(u y)\right\|_{Y}^{p}}{V_{n} u^{p+1}}\right. & \mathrm{d} x \mathrm{~d} y \mathrm{~d} u)^{\frac{1}{p}} \\
& \lesssim \sqrt{p n} C_{p}(Y) \beta_{p}(Y)^{7} \log \left(\beta_{p}(Y) n\right) \sum_{j=1}^{n}\left(\int_{\mathbb{R}^{n}}\left\|\frac{\partial f}{\partial x_{j}}(x)\right\|_{Y}^{p} \mathrm{~d} x\right)^{\frac{1}{p}} .
\end{aligned}
$$

Note that Theorem 19 follows from Theorem 40. Indeed, if $\left(Y,\|\cdot\|_{Y}\right)$ is a UMD Banach space with $\beta=\beta(Y)$ then by Lemma 32 there exists a universal constant $\kappa \in(0, \infty)$ such that if we set $p=\kappa \beta$ then $C_{p}(Y) \lesssim 1$ (in particular we necessarily have $\kappa \beta \geqslant 2$ ). Moreover, by (49) we have $\beta_{p}(Y) \lesssim \beta^{2}$. To deduce Theorem 19, take $f: \mathbb{R}^{n} \rightarrow Y$ that is Lipschitz and compactly supported. By convolving $f$ with a smooth bump function whose support has small diameter we may also assume that $f$ is smooth. It now follows from Theorem 40 that

$$
\begin{aligned}
\left(\iiint_{\mathbb{R}^{n} \times B^{n} \times(0, \infty)}\right. & \left.\frac{\left\|f^{x}(u y)-P_{u}^{1} f^{x}(u y)\right\|_{Y}^{\kappa \beta}}{V_{n} u^{\kappa \beta+1}} \mathrm{~d} x \mathrm{~d} y \mathrm{~d} u\right)^{\frac{1}{\kappa \beta}} \\
& \lesssim \beta^{\frac{29}{2}} \sqrt{n} \log (\beta n) \sum_{j=1}^{n}\left(\int_{\mathbb{R}^{n}}\left\|\frac{\partial f}{\partial x_{j}}(x)\right\|_{Y}^{\kappa \beta} \mathrm{d} x\right)^{\frac{1}{\kappa \beta}} \leqslant \beta^{15} n^{\frac{5}{2}}(\operatorname{vol}(\operatorname{supp}(f)))^{\frac{1}{\kappa \beta}}\|f\|_{\text {Lip }},
\end{aligned}
$$

where we used the fact that, due to (8), for every $x \in \mathbb{R}^{n}$ and $j \in\{1, \ldots, n\}$ we have

$$
\left\|\frac{\partial f}{\partial x_{j}}(x)\right\|_{Y}=\lim _{t \rightarrow 0} \frac{\left\|f\left(x+t e_{j}\right)-f(x)\right\|_{Y}}{|t|} \leqslant\|f\|_{\text {Lip }}\left\|e_{j}\right\|_{X} \stackrel{(8)}{\leqslant}\|f\|_{\operatorname{Lip}} \sqrt{n} .
$$

Proof of Theorem 40. For every $s \in(0, \infty)$ let $v_{s}$ be the measure on $(0, \infty) \times \mathbb{R}^{n} \times B^{n}$ whose density is given by

$$
\forall(u, x, y) \in(0, \infty) \times \mathbb{R}^{n} \times B^{n}, \quad \varphi_{s}(u, x, y) \stackrel{\text { def }}{=} \frac{1}{V_{n}|u|^{p s+1}} .
$$


Define a linear operator $\mathscr{S}: H_{s, p}\left(\mathbb{R}^{n}, Y\right) \rightarrow L_{p}\left(v_{s}, Y\right)$ by setting for every $f \in H_{s, p}\left(\mathbb{R}^{n}, Y\right)$,

$$
\forall(u, x, y) \in(0, \infty) \times \mathbb{R}^{n} \times B^{n}, \quad \mathscr{S} f(u, x, y) \stackrel{\text { def }}{=} f^{x}(u y)-P_{u}^{1} f^{x}(u y),
$$

where we recall the notations (9) and (15) for $f^{x}$ and $P_{u}^{1}$, respectively. In what follows we let $M_{s}$ denote the norm of $\mathscr{S}$ as an operator from $H_{s, p}\left(\mathbb{R}^{n}, Y\right)$ to $L_{p}\left(v_{s}, Y\right)$, i.e.,

$$
\forall s \in(0, \infty), \quad M_{s} \stackrel{\text { def }}{=}\|\mathscr{S}\|_{H_{s, p}\left(\mathbb{R}^{n}, Y\right) \rightarrow L_{p}\left(v_{s}, Y\right)} .
$$

Suppose that $s, \sigma, \theta \in \mathbb{R}$ satisfy

$$
(s, \sigma, \theta) \in(0,1) \times(1,2) \times(0,1) \quad \text { and } \quad 1=(1-\theta) s+\theta \sigma .
$$

Then $\varphi_{1}=\varphi_{s}^{1-\theta} \varphi_{\sigma}^{\theta}$, so by Stein's interpolation theorem [71, Theorem 2] for every $f$ in the complex interpolation space $\left[H_{s, p}\left(\mathbb{R}^{n}, Y\right), H_{\sigma, p}\left(\mathbb{R}^{n}, Y\right)\right]_{\theta}$ we have

$$
\begin{aligned}
\left(\iiint_{\mathbb{R}^{n} \times B^{n} \times(0, \infty)} \frac{\left\|f^{x}(u y)-P_{u}^{1} f^{x}(u y)\right\|_{Y}^{p}}{V_{n} u^{p+1}} \mathrm{~d} x \mathrm{~d} y \mathrm{~d} u\right)^{\frac{1}{p}} \\
\stackrel{(138) \wedge(139)}{=}\|\mathscr{S} f\|_{L_{p}\left(v_{1}, Y\right)} \leqslant M_{s}^{1-\theta} M_{\sigma}^{\theta}\|f\|_{\left[H_{s, p}\left(\mathbb{R}^{n}, Y\right), H_{\sigma, p}\left(\mathbb{R}^{n}, Y\right)\right]_{\theta}} .
\end{aligned}
$$

We note that Stein's interpolation theorem is stated in [71] for real-valued function spaces, but the standard proofs of this theorem (see also [74] or [9, Section 5.4]) work without additional effort for vector-valued spaces as well, which is what we are using here. Alternatively, the vector-valued setting is treated explicitly by Calderón in [18, Section 13.6]. Every $f \in W_{1, p}\left(\mathbb{R}^{n}, Y\right)$ satisfies

$$
\|f\|_{\left[H_{s, p}\left(\mathbb{R}^{n}, Y\right), H_{\sigma, p}\left(\mathbb{R}^{n}, Y\right)\right]_{\theta}} \stackrel{(*)}{\lesssim} \beta_{p}(Y)\|f\|_{H_{1, p}\left(\mathbb{R}^{n}, Y\right)} \stackrel{(* *)}{\lesssim} \beta_{p}(Y)^{3}\|f\|_{W_{1, p}\left(\mathbb{R}^{n}, Y\right)} .
$$

where in $(*)$ we used Lemma 34 and in $(* *)$ we used Lemma 35. Hence,

$$
\begin{aligned}
\left(\iiint_{\mathbb{R}^{n} \times B^{n} \times(0, \infty)} \frac{\left\|f^{x}(u y)-P_{u}^{1} f^{x}(u y)\right\|_{Y}^{p}}{V_{n} u^{p+1}} \mathrm{~d} x \mathrm{~d} y \mathrm{~d} u\right)^{\frac{1}{p}} & \left(\inf _{\substack{(109) \wedge(140) \wedge(141) \\
\lesssim}} \beta_{p}(Y)^{3}\left(M_{\substack{(s, \sigma, \theta) \in(0,1) \times(1,2) \times(0,1) \\
(1-\theta) s+\theta \sigma=1}}^{1-\theta} M_{\sigma}^{\theta}\right) \sum_{j=1}^{n}\left(\int_{\mathbb{R}^{n}}\left\|\frac{\partial f}{\partial x_{j}}(x)\right\|_{Y}^{p} \mathrm{~d} x\right)^{\frac{1}{p}} .\right.
\end{aligned}
$$

Corollary 39 asserts that

$$
\forall(s, \sigma) \in(0,1) \times(1,2), \quad M_{s} \lesssim \frac{\sqrt{p n} C_{p}(Y) \beta_{p}(Y)^{4}}{s(1-s)} \quad \text { and } \quad M_{\sigma} \lesssim \frac{\sqrt{p} n^{\frac{3}{2}} C_{p}(Y) \beta_{p}(Y)^{6}}{(\sigma-1)(2-\sigma)} .
$$

Hence,

$$
\begin{aligned}
\inf _{\substack{(s, \sigma, \theta) \in(0,1) \times(1,2) \times(0,1) \\
(1-\theta) s+\theta \sigma=1}} M_{s}^{1-\theta} M_{\sigma}^{\theta} & \lesssim \inf _{\substack{(s, \sigma, \theta) \in(0,1) \times(1,2) \times(0,1) \\
(1-\theta) s+\theta \sigma=1}}\left(\frac{\sqrt{p n} C_{p}(Y) \beta_{p}(Y)^{4}}{s(1-s)}\right)^{1-\theta}\left(\frac{\sqrt{p} n^{\frac{3}{2}} C_{p}(Y) \beta_{p}(Y)^{6}}{(\sigma-1)(2-\sigma)}\right)^{\theta} \\
& \lesssim \sqrt{p n} C_{p}(Y) \beta_{p}(Y)^{4} \log \left(\beta_{p}(Y) n\right)
\end{aligned}
$$


where (143) arises by choosing $s=1-1 / \log \left(\beta_{p}(Y) n\right)$ and $\sigma=2-1 / \log \left(\beta_{p}(Y) n\right)$, in which case necessarily $\theta=1 / \log \left(\beta_{p}(Y) n\right)$. We note that these choices essentially yield the best possible estimate in (143) (up to constant factors), but one could also choose here, say, $s=1 / 2$ and $\sigma=3 / 2$, yielding a worse dependence on $n$ which is of lesser importance for our present purposes. A substitution of (143) into (142) yields the desired estimate (137).

\section{Acknowledgements}

We are grateful to Charles Fefferman for allowing us to include the contents of Section 2.2. We also thank Mark Veraar for helpful pointers to the literature and we are grateful to the anonymous referee for a careful reading and detailed comments that improved the presentation.

Part of this work was completed during a visit of the three authors to the MSRI Quantitative Geometry program. Another part of this work was completed when A. N. was visiting Université Pierre et Marie Curie, Paris, France.

\section{Added in proof}

In the forthcoming work [39], Question 8 is resolved positively by showing that Theorem 2 holds true for any uniformly convex target $Y$ (in which case the parameter $\beta$ is replaced by a quantity that depends on the modulus of uniform convexity of $Y$ ). Also, the dependence on $n$ that appears in (7) is improved in [39]. This is achieved in [39] by following the vector-valued Littlewood-Paley strategy that we introduced here to bound $r^{X \rightarrow Y}(\varepsilon)$, but while implementing it via a method that differs markedly from the argument that appears in the present work. Specifically, [39] follows more closely Bourgain's original strategy [13] for proving his discretization theorem, though with major differences. In particular, the proof in [39] even yields a new approach to Dorronsoro's influential classical work [26] in the scalar-valued setting.

\section{References}

[1] D. J. Aldous. Unconditional bases and martingales in $L_{p}(F)$. Math. Proc. Cambridge Philos. Soc., 85(1):117-123, 1979. 31

[2] J. Azzam and R. Schul. A quantitative metric differentiation theorem. Proc. Amer. Math. Soc., 142(4):1351-1357, 2014. 5

[3] R. Bañuelos and K. Bogdan. Lévy processes and Fourier multipliers. J. Funct. Anal., 250(1):197213, 2007. 27

[4] R. Bañuelos and P. J. Méndez-Hernández. Space-time Brownian motion and the Beurling-Ahlfors transform. Indiana Univ. Math. J., 52(4):981-990, 2003. 21

[5] F. Barthe, O. Guédon, S. Mendelson, and A. Naor. A probabilistic approach to the geometry of the $l_{p}^{n}$-ball. Ann. Probab., 33(2):480-513, 2005. 8 
QuAntitative AfFine ApProximation FOR UMD TARgEts

[6] S. Bates, W. Johnson, J. Lindenstrauss, D. Preiss, and G. Schechtman. Affine approximation of Lipschitz functions and nonlinear quotients. Geometric and Functional Analysis, 9:1092-1127, 1999. $3,4,5,6$

[7] Y. Benyamini. The uniform classification of Banach spaces. In Texas functional analysis seminar 1984-1985 (Austin, Tex.), Longhorn Notes, pages 15-38. Univ. Texas Press, Austin, TX, 1985. 4

[8] Y. Benyamini and J. Lindenstrauss. Geometric nonlinear functional analysis. Vol. 1, volume 48 of American Mathematical Society Colloquium Publications. American Mathematical Society, Providence, RI, 2000. 4

[9] J. Bergh and J. Löfström. Interpolation spaces. An introduction. Springer-Verlag, Berlin-New York, 1976. Grundlehren der Mathematischen Wissenschaften, No. 223. 33, 37, 41

[10] E. Berkson and T. A. Gillespie. Spectral decompositions and harmonic analysis on UMD spaces. Studia Math., 112(1):13-49, 1994. 20

[11] J. Bourgain. Some remarks on Banach spaces in which martingale difference sequences are unconditional. Ark. Mat., 21(2):163-168, 1983. 6

[12] J. Bourgain. Vector-valued singular integrals and the $H^{1}$-BMO duality. In Probability theory and harmonic analysis (Cleveland, Ohio, 1983), volume 98 of Monogr. Textbooks Pure Appl. Math., pages 1-19. Dekker, New York, 1986. 20, 29

[13] J. Bourgain. Remarks on the extension of Lipschitz maps defined on discrete sets and uniform homeomorphisms. In Geometrical aspects of functional analysis (1985/86), volume 1267 of Lecture Notes in Math., pages 157-167. Springer, Berlin, 1987. 2, 4, 42

[14] D. Burkholder. Martingales and singular integrals in Banach spaces. In Handbook of the geometry of Banach spaces, volume 1, pages 233-269. 2001. 3

[15] D. L. Burkholder. A geometric condition that implies the existence of certain singular integrals of Banach-space-valued functions. In Conference on harmonic analysis in honor of Antoni Zygmund, Vol. I, II (Chicago, Ill., 1981), Wadsworth Math. Ser., pages 270-286. Wadsworth, Belmont, CA, 1983. 36

[16] D. L. Burkholder. Boundary value problems and sharp inequalities for martingale transforms. Ann. Probab., 12(3):647-702, 1984. 27

[17] D. L. Burkholder. Martingales and Fourier analysis in Banach spaces. In Probability and analysis (Varenna, 1985), volume 1206 of Lecture Notes in Math., pages 61-108. Springer, Berlin, 1986. 19

[18] A.-P. Calderón. Intermediate spaces and interpolation, the complex method. Studia Math., 24:113190, 1964. 41

[19] J. Cheeger. Quantitative differentiation: a general formulation. Comm. Pure Appl. Math., 65(12):1641-1670, 2012. 4 
[20] J. Cheeger, B. Kleiner, and A. Naor. Compression bounds for Lipschitz maps from the Heisenberg group to $L_{1}$. Acta Math., 207(2):291-373, 2011. 4

[21] P. Clément, B. de Pagter, F. A. Sukochev, and H. Witvliet. Schauder decomposition and multiplier theorems. Studia Math., 138(2):135-163, 2000. 20

[22] G. David and S. Semmes. Singular integrals and rectifiable sets in $\mathbf{R}^{n}$ : Beyond Lipschitz graphs. Astérisque, (193):152, 1991. 5

[23] G. David and S. Semmes. Analysis of and on uniformly rectifiable sets, volume 38 of Mathematical Surveys and Monographs. American Mathematical Society, Providence, RI, 1993. 5

[24] G. David and S. Semmes. Regular mappings between dimensions. Publ. Mat., 44(2):369-417, 2000. 5

[25] L. Deleaval, O. Guédon, and B. Maurey. Dimension free bounds for the Hardy-Littlewood maximal operator associated to convex sets. Preprint, available at http://arxiv.org/abs/1602.02015, 2016. 25

[26] J. Dorronsoro. A characterization of potential spaces. Proc. Amer. Math. Soc., 95(1):21-31, 1985. $4,5,9,15,42$

[27] A. Eskin, D. Fisher, and K. Whyte. Coarse differentiation of quasi-isometries I: Spaces not quasi-isometric to Cayley graphs. Ann. of Math. (2), 176(1):221-260, 2012. 4

[28] W. Feller. An introduction to probability theory and its applications. Vol. II. Second edition. John Wiley \& Sons Inc., New York, 1971. 31

[29] T. Figiel and G. Pisier. Séries aléatoires dans les espaces uniformément convexes ou uniformément lisses. C. R. Acad. Sci. Paris Sér. A, 279:611-614, 1974. 31

[30] T. Figiel and P. Wojtaszczyk. Special bases in function spaces. In Handbook of the geometry of Banach spaces, Vol. I, pages 561-597. North-Holland, Amsterdam, 2001. 20

[31] O. Friedland and O. Guédon. Random embedding of $\ell_{p}^{n}$ into $\ell_{r}^{N}$. Math. Ann., 350(4):953-972, 2011. 32

[32] D. J. H. Garling. Brownian motion and UMD-spaces. In Probability and Banach spaces (Zaragoza, 1985), volume 1221 of Lecture Notes in Math., pages 36-49. Springer, Berlin, 1986. 21, 36

[33] D. J. H. Garling. Random martingale transform inequalities. In Probability in Banach spaces 6 (Sandbjerg, 1986), volume 20 of Progr. Probab., pages 101-119. Birkhäuser Boston, Boston, MA, 1990. 19

[34] S. Geiss. A counterexample concerning the relation between decoupling constants and UMDconstants. Trans. Amer. Math. Soc., 351(4):1355-1375, 1999. 19 
[35] O. Giladi, A. Naor, and G. Schechtman. Bourgain's discretization theorem. Ann. Fac. Sci. Toulouse Math. (6), 21(4):817-837, 2012. 2, 4

[36] S. Guerre-Delabrière. Some remarks on complex powers of $(-\Delta)$ and UMD spaces. Illinois $J$. Math., 35(3):401-407, 1991. 26

[37] R. F. Gundy and N. T. Varopoulos. Les transformations de Riesz et les intégrales stochastiques. $C$. R. Acad. Sci. Paris Sér. A-B, 289(1):A13-A16, 1979. 21

[38] Y.-S. Han and Y. Meyer. A characterization of Hilbert spaces and the vector-valued Littlewood-Paley theorem. Methods Appl. Anal., 3(2):228-234, 1996. 39

[39] T. Hytönen and A. Naor. Heat flow and quantitative differentiation. Preprint available at https: //arxiv.org/abs/1608.01915, 2016. 42

[40] T. Hytönen, J. van Neerven, M. Veraar, and L. Weis. Analysis in Banach spaces. Vol. I. Monograph in preparation, draft available at http://fa.its.tudelft.nl/ neerven/ABS/volume1_ Hytonen_Neerven_Veraar_Weis.pdf, 2015. 29

[41] T. P. Hytönen. Aspects of probabilistic Littlewood-Paley theory in Banach spaces. In Banach spaces and their applications in analysis, pages 343-355. Walter de Gruyter, Berlin, 2007. 21, 26

[42] T. P. Hytönen. Littlewood-Paley-Stein theory for semigroups in UMD spaces. Rev. Mat. Iberoam., 23(3):973-1009, 2007. 21

[43] T. P. Hytönen. On the norm of the Beurling-Ahlfors operator in several dimensions. Canad. Math. Bull., 54(1):113-125, 2011. 21

[44] T. Iwaniec and G. Martin. Riesz transforms and related singular integrals. J. Reine Angew. Math., 473:25-57, 1996. 35

[45] F. John. Extremum problems with inequalities as subsidiary conditions. In Studies and essays presented to R. Courant on his 60th birthday, pages 187-204. Interscience Publishers, Inc., 1948. 6

[46] W. B. Johnson, J. Lindenstrauss, and G. Schechtman. Banach spaces determined by their uniform structures. Geom. Funct. Anal., 6(3):430-470, 1996. 4

[47] W. B. Johnson and G. Schechtman. Embedding $l_{p}^{m}$ into $l_{1}^{n}$. Acta Math., 149(1-2):71-85, 1982. 32

[48] P. W. Jones. Square functions, Cauchy integrals, analytic capacity, and harmonic measure. In Harmonic analysis and partial differential equations (El Escorial, 1987), volume 1384 of Lecture Notes in Math., pages 24-68. Springer, Berlin, 1989. 5

[49] P. W. Jones. Rectifiable sets and the traveling salesman problem. Invent. Math., 102(1):1-15, 1990. 5

[50] J.-P. Kahane. Some random series of functions, volume 5 of Cambridge Studies in Advanced Mathematics. Cambridge University Press, Cambridge, second edition, 1985. 20 
[51] I. Karatzas and S. E. Shreve. Brownian motion and stochastic calculus, volume 113 of Graduate Texts in Mathematics. Springer-Verlag, New York, second edition, 1991. 21, 22, 24

[52] S. Kwapień. Isomorphic characterizations of inner product spaces by orthogonal series with vector valued coefficients. Studia Math., 44:583-595, 1972. Collection of articles honoring the completion by Antoni Zygmund of 50 years of scientific activity, VI. 39

[53] M. Ledoux and M. Talagrand. Probability in Banach spaces, volume 23 of Ergebnisse der Mathematik und ihrer Grenzgebiete (3) [Results in Mathematics and Related Areas (3)]. Springer-Verlag, Berlin, 1991. Isoperimetry and processes. 19

[54] J. R. Lee and P. Raghavendra. Coarse differentiation and multi-flows in planar graphs. Discrete Comput. Geom., 43(2):346-362, 2010. 4

[55] S. Li. Coarse differentiation and quantitative nonembeddability for Carnot groups. J. Funct. Anal., 266(7):4616-4704, 2014. 4

[56] S. Li. Markov convexity and nonembeddability of the Heisenberg group. To appear in Ann. Inst. Fourier. Available at http://arxiv.org/abs/1404.6751, 2014. 4

[57] S. Li and A. Naor. Discretization and affine approximation in high dimensions. Israel J. Math., 197(1):107-129, 2013. 2, 3, 4, 6, 12

[58] M. B. Marcus and G. Pisier. Characterizations of almost surely continuous $p$-stable random Fourier series and strongly stationary processes. Acta Math., 152(3-4):245-301, 1984. 32

[59] T. Martínez, J. L. Torrea, and Q. Xu. Vector-valued Littlewood-Paley-Stein theory for semigroups. Adv. Math., 203(2):430-475, 2006. 6, 20

[60] J. Matoušek. On embedding trees into uniformly convex Banach spaces. Israel J. Math., 114:221237, 1999. 4

[61] B. Maurey. Système de Haar. In Séminaire Maurey-Schwartz 1974-1975: Espaces Lsup p, applications radonifiantes et géométrie des espaces de Banach, Exp. Nos. I et II, pages 26 pp. (erratum, p. 1). Centre Math., École Polytech., Paris, 1975. 31

[62] B. Maurey. Type, cotype and $K$-convexity. In Handbook of the geometry of Banach spaces, Vol. 2, pages 1299-1332. North-Holland, Amsterdam, 2003. 31

[63] B. Maurey and G. Pisier. Séries de variables aléatoires vectorielles indépendantes et propriétés géométriques des espaces de Banach. Studia Math., 58(1):45-90, 1976. 31

[64] M. Mendel and A. Naor. Markov convexity and local rigidity of distorted metrics. J. Eur. Math. Soc. (JEMS), 15(1):287-337, 2013. 4

[65] A. Naor. Geometric problems in non-linear functional analysis. Master's thesis, Hebrew University, 1998. 4 
QuAntitative AfFine ApProximation FOR UMD TARgEts

[66] G. Pisier. Martingales with values in uniformly convex spaces. Israel J. Math., 20(3-4):326-350, 1975. 31

[67] G. Pisier. Un exemple concernant la super-réflexivité. In Séminaire Maurey-Schwartz 1974-1975: Espaces $L^{p}$ applications radonifiantes et géométrie des espaces de Banach, Annexe No. 2, page 12. Centre Math. École Polytech., Paris, 1975. 3, 6

[68] G. Pisier. On the dimension of the $l_{p}^{n}$-subspaces of Banach spaces, for $1 \leqslant p<2$. Trans. Amer. Math. Soc., 276(1):201-211, 1983. 31, 32

[69] Y. Qiu. On the UMD constants for a class of iterated $L_{p}\left(L_{q}\right)$ spaces. J. Funct. Anal., 263(8):24092429, 2012. 6

[70] G.-C. Rota. An "Alternierende Verfahren" for general positive operators. Bull. Amer. Math. Soc., 68:95-102, 1962. 20

[71] E. M. Stein. Interpolation of linear operators. Trans. Amer. Math. Soc., 83:482-492, 1956. 41

[72] E. M. Stein. Singular integrals and differentiability properties of functions. Princeton Mathematical Series, No. 30. Princeton University Press, Princeton, N.J., 1970. 5, 11, 36

[73] E. M. Stein. Topics in harmonic analysis related to the Littlewood-Paley theory. Annals of Mathematics Studies, No. 63. Princeton University Press, Princeton, N.J., 1970. 20

[74] E. M. Stein and G. Weiss. Interpolation of operators with change of measures. Trans. Amer. Math. Soc., 87:159-172, 1958. 41

[75] H. Triebel. Theory of function spaces. II, volume 84 of Monographs in Mathematics. Birkhäuser Verlag, Basel, 1992. 15

[76] J. M. A. M. van Neerven. Stochastic evolution equations. Lecture notes of the Internet Seminar 2007/08 (version May 07, 2010). Available at http://fa.its.tudelft.nl/〜neerven/ publications/papers/ISEM.pdf, 2010. 21, 22

[77] J. M. A. M. van Neerven, M. C. Veraar, and L. Weis. Stochastic integration in UMD Banach spaces. Ann. Probab., 35(4):1438-1478, 2007. 21

[78] J. M. A. M. van Neerven and L. Weis. Stochastic integration of functions with values in a Banach space. Studia Math., 166(2):131-170, 2005. 21

[79] M. Veraar. Embedding results for $\gamma$-spaces. In Recent trends in analysis. Proceedings of the conference in honor of Nikolai Nikolski on the occasion of his 70th birthday, Bordeaux, France, August 31 - September 2, 2011, pages 209-219. Bucharest: The Theta Foundation, 2013. 38 


\section{Tuomas Hytönen, SeAn Li, And Assaf NaOR}

\section{AUTHORS}

Tuomas Hytönen

University of Helsinki

Helsinki, Finland

tuomas [dot] hytonen@helsinki [dot] fi

http://wiki.helsinki.fi/pages/viewpage . action?pageId=31427518

Sean Li

University of Chicago, Chicago IL, USA

seanli [dot] math@uchicago [dot] edu

https://sites.google.com/site/seanlimath/

\section{Assaf Naor}

Princeton University

Princeton NJ, USA

naor@math [dot] princeton [dot] edu

https://web.math.princeton.edu/ naor/ 